\title{
BROMELIAD FLORA OF OAXACA, MEXICO: RICHNESS AND DISTRIBUTION
}

\author{
Adolfo Espejo-Serna ${ }^{1}$, Ana Rosa López-Ferrari ${ }^{1}$,Nancy Martínez-Correa ${ }^{1}$ and \\ Valeria Angélica Pulido-Esparza ${ }^{2}$ \\ ${ }^{1}$ Universidad Autónoma Metropolitana-Iztapalapa, \\ División de Ciencias Biológicas y de la Salud, Departamento de Biología, \\ Herbario Metropolitano, Apdo. postal 55-535, 09340 \\ México, D.F., México. aes@xanum.uam.mx \\ ${ }^{2}$ El Colegio de la Frontera Sur - San Cristóbal de las Casas, \\ Laboratorio de Análisis de Información Geográfica y Estadística, \\ Chiapas, México.feliscatus@yahoo.com
}

\begin{abstract}
The current knowledge of the bromeliad flora of the state of Oaxaca, Mexico is presented. Oaxaca is the Mexican state with the largest number of bromeliad species. Based on the study of 2,624 herbarium specimens corresponding to 1,643 collections, and a detailed bibliographic revision, we conclude that the currently known bromeliad flora for Oaxaca comprises 172 species and 15 genera. All Mexican species of the genera Bromelia, Fosterella, Greigia, Hohenbergiopsis, Racinaea, and Vriesea are represented in the state. Aechmea nudicaulis, Bromelia hemisphaerica, Catopsis nitida, C. oerstediana, C. wawranea, Pitcairnia schiedeana, P. tuerckheimii, Racinaea adscendens, Tillandsia balbisiana, T. belloensis, T. brachycaulos, T. compressa, T. dugesii, T. foliosa, T. flavobracteata, T. limbata, T. maritima, T. ortgiesiana, T. paucifolia, T. pseudobaileyi, T. rettigiana, T. utriculata, T. x marceloi, Werauhia pycnantha, and W. nutans are recorded for the first time from Oaxaca. Collections from 226 (of 570) municipalities and all 30 districts of the state were studied. Among the vegetation types occurring in Oaxaca, oak forest is the richest with 83 taxa, followed by tropical deciduous forest with 74, and cloud forest with 73 species. Species representation and distribution in Oaxaca are analyzed in detail. We also provide a comparison with bromeliad floras of the states of Chiapas, Guerrero, Puebla and Veracruz. The analysis of the species and collections by altitudinal intervals shows that the highest numbers of both ocurre between 1,500 and 2,000 m, with the number of species markedly decreasing above 2,500 m.
\end{abstract}

Key words: Bromeliaceae, distribution, diversity, endemism, flora, Mexico, Oaxaca. 


\section{RESUMEN}

Se presenta el estado actual del conocimiento de la flora bromeliológica del estado de Oaxaca, México. La entidad ocupa el primer lugar en el país en cuanto a número de especies de Bromeliaceae se refiere. Los resultados obtenidos de la revisión de 2,624 ejemplares herborizados, correspondientes a 1,643 colectas, así como la revisión de bibliografía especializada, muestran que en el estado están presentes 172 especies agrupadas en 15 géneros. Bromelia, Fosterella, Greigia, Hohenbergiopsis, Racinaea y Vriesea tienen representados a todos sus taxa mexicanos. Se registran por primera vez para el estado: Aechmea nudicaulis, Bromelia hemisphaerica, Catopsis nitida, C. oerstediana, C. wawranea, Pitcairnia schiedeana, P. tuerckheimii, Racinaea adscendens, Tillandsia balbisiana, T. belloensis, T. brachycaulos, T. compressa, T. dugesii, T. foliosa, T. flavobracteata, T. limbata, T. maritima, T. ortgiesiana, T. paucifolia, T. pseudobaileyi, T. rettigiana, T. utriculata, T. x marceloi, Werauhia pycnantha y W. nutans. Se registraron colectas para 226 municipios de los 570 y para el total de los distritos (30) en los que está dividido políticamente el estado. Se hizo una comparación de la flora bromeliológica de Oaxaca con la de Chiapas, Guerrero, Puebla y Veracruz. De los tipos de vegetación presentes, el Bosque de Quercus es el que presenta mayor riqueza de taxa (83), seguido por el Bosque Tropical Caducifolio (74) y el Bosque Mesófilo de Montaña (73). El análisis del número de especies y de colecciones por intervalo altitudinal muestra que las cantidades más altas, tanto de colectas como de especies, se concentran entre los 1,500 y los 2,000 m s.n.m., disminuyendo claramente por arriba de los 2,500 m.

Palabras clave: Bromeliaceae, distribución, diversidad, endemismo, flora, México, Oaxaca.

\section{INTRODUCTION}

The Bromeliaceae contain approximately 3,086 species in 56 genera (Luther, 2006); in Mexico there are 18 genera and 342 species (Espejo et al., 2004). The family is well distributed across the country, as well as in all the vegetation types sensu Rzedowski (1978). It is a valuable component of many different plant associations, not only for the shear abundance of its species but also for the ecological role they play.

The family is important on many levels. Ecologically they are central epiphytic components in various ecosystems, possessing various physiological adaptations for atmospheric stress and serving as unique habitats for many animal species. Economically, bromeliads are valuable because of their ornamental and edible species. 
Their floristic importance relates to their wide distribution throughout the Neotropics and the high number of narrowly endemic species. Despite these factors, the family has received relatively little attention with regard to floristic studies.

Although there are various regional treatments for the Mexican Bromeliaceae (e.g., McVaugh (1989), Utley and Burt-Utley (1994), Victoria (2001), Arellano Mijangos (2002), Pulido-Esparza et al. (2004), Espejo et al. (2005), and Ramírez-Morillo et al. (2004)), the only two contributions of national scope are those of Smith and Downs (1974; 1977; 1979) and Espejo et al. (2004). The purpose of this article is to provide an updated list of the members of the family in Oaxaca, with emphasis on aspects of endemism and geographic distribution.

\section{Study area}

Oaxaca is located in the southern portion of Mexico between $15^{\circ} 39^{\prime}$ and $18^{\circ} 09^{\prime} \mathrm{N}$ latitude and $93^{\circ} 52^{\prime}$ and $98^{\circ} 32^{\prime} \mathrm{W}$ longitude and covers an area of 93,343 $\mathrm{km}^{2}, 4.8 \%$ of the country's surface (Anonymous, 2004) (Fig. 1). The state is politically divided into 30 districts and 570 municipios that include more than 10,000 towns and villages (Anonymous, 2004). It also is divided economically into eight regions: Cañada, Costa, Istmo, Mixteca, Papaloapan, Sierra Norte, Sierra Sur, and Valles Centrales (Anonymous, 2004).

Five physiographic regions are distinguished in Oaxaca (Anonymous, 2004): 1) Sierra Madre del Sur, 2) Cordillera Centroamericana, 3) Llanura Costera del Golfo sur, 4) Eje Neovolcánico, and 5) Sierras de Chiapas y Guatemala. Elevation varies from sea level along the coastal plains at the Pacific Ocean to 3,000 $\mathrm{m}$ in the "Sierra Madre de Oaxaca" (Ortíz-Pérez et al., 2004).

Also, five climatic regions are distinguished in Oaxaca (Trejo, 2004): 1) Cuenca del Balsas, 2) Sierra Madre del Sur y Llanura Costera, 3) Mixteca y Valles de Oaxaca, 4) Sierra Madre de Chiapas y Llanura Costera, and 5) Sierra Madre Oriental.

The climatic and physiographic variation is reflected in the diversity of vegetation communities that exists in Oaxaca, and these communities possess the greatest biological diversity of all Mexican states. Within the state the following types of vegetation (sensu Rzedowski, 1978) have been reported: Bosque Tropical Perennifolio, Bosque Tropical Subcaducifolio, Bosque Tropical Caducifolio, Bosque Espinoso, Matorral Xerófilo, Bosque de Quercus, Bosque de Coníferas, Bosque Mesófilo de Montaña, Palmar, Bosque de Galería,Vegetación Acuática y Subacuática. 


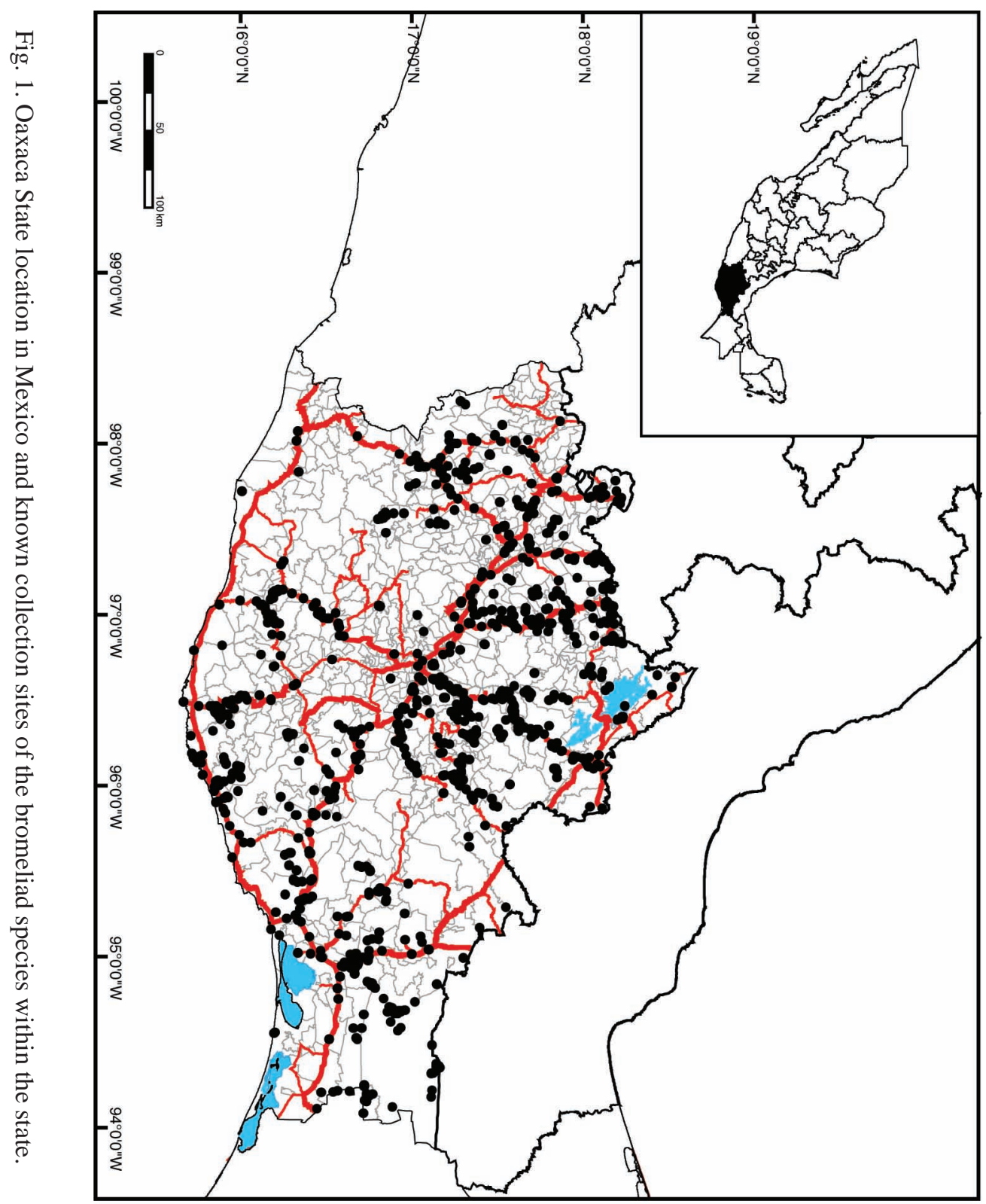




\section{METHODS}

From June of 1997 until June of 2006, Oaxacan specimens from the following herbaria were examined, identified, annotated, and captured in a database: B, BM, BR, C, CHAP, CICY, CIIDIR, CODAGEM, ECON, ENCB, F, FCME, FI, GH, GOET, HEID, IBUG, IEB, K, LG, LL, M, MA, MEXU, MICH, MO, NY, OXF, P, SEL, SERO, TEX, UAMIZ, UC, UNICACH, US, VT, WU, XAL, and Z. The information from these specimens was enhanced by a carefull revision of pertinent bibliographic material (Baker, 1889; Mez, 1896; Smith, 1961; Smith \& Downs, 1974; 1977; 1979; Grant, 1995 a; b; Palací, 1997; Till, 1990, 1992).

In order to analyze the distribution of the species, geographic coordinates were obtained from the labels of the examined specimens. These were checked and corrected if necessary, and subsequently, the data were processed in the program Arc View GIS 8 (Anonymous, 2003). In instances where such information was lacking, the location was obtained from topographic 1:250,000 scale maps (Instituto Nacional de Estadística, Geografía e Informática (INEGI) maps D14-3, E14-6, E148, E14-9, E14-12, E15-7 and E15-10), and from the web site http://mapserver.inegi. gob.mx/dsist/ahl2003/index.html?c=424.

Two data matrices for species presence-absence were constructed, one relative to the vegetation types and other relative to the adjacent states (Guerrero, Chiapas, Puebla, and Veracruz). These matrices were used to obtain Jaccard indices of similarity. Also, cluster analyses were done in order to determine the affinities of the Oaxacan bromeliad flora with respect to that of the adjacent states and to evaluate the similarity of the flora with respect to the types of vegetation present in the state. These analyses were conducted using the program NTSYSpc2.0 (Rohlf, 1998).

A species accumulation curve was generated to assess the knowledge and extent of exploration of the state's bromeliad flora. The collection year information used to generate this curve was obtained directly from herbarium labels, using as a sample the total number of species recorded each five years. The starting point was 1840 , the year of the first known collection of Bromeliaceae in Oaxaca. In addition, a rarefaction curve, taking into account the abundance of each taxon, was generated (Colwell \& Coddington, 1994) to compare the data obtained in the accumulation curve with that expected using Chao1 and Jacknife 2 estimators (Chao, 1984; Colwell \& Coddington, 1994). The algorithms were calculated with the program EstimatesS Ver. 7 (Colwell, 2004). 


\section{RESULTS AND DISCUSSION}

After examining 2,624 herbarium sheets, corresponding to 1,643 collections, we can say now that the known bromeliad flora of the state is composed by 172 species and 15 genera (appendix). This figure represents $50.29 \%$ of the 342 species of the family registered for the country (Espejo et al., 2004). In contrast, the two previous publications that provide integral information about the bromeliad Flora of Oaxaca, Flora Neotropica (Smith \& Downs, 1974, 1977, 1979) and the Checklist of Mexican Bromeliaceae (Espejo et al., 2004), reported 14 genera, 88 species based on 213 collections and 15 genera, 135 species based on 1482 collections, respectively.

The genera with the greatest number of species in the area are Tillandsia (101 spp.) and Hechtia (20 spp.). In contrast, Billbergia, Fosterella, Guzmania and Hohenbergiopsis each possess a single species. Bromelia, Fosterella, Greigia, Hohenbergiopsis, Racinaea, and Vriesea have all their Mexican taxa represented in Oaxaca. The following species are reported from the state for the first time: Aechmea nudicaulis, Bromelia hemisphaerica, Catopsis nitida, C. oerstediana, C. wawranea, Pitcairnia schiedeana, P. tuerckheimii, Racinaea adscendens, Tillandsia balbisiana, T. belloensis, T. brachycaulos, T. compressa, T. dugesii, T. foliosa, T. flavobracteata, T. limbata, T. maritima, T. ortgieseana, T. paucifolia, T. pseudobaileyi, T. rettigiana, T. utriculata, T. x marceloi, Werauhia nutans, and W. pycnantha.

Oaxaca, with 43 narrowly endemic species (Appendix; Figs. 10, 11, 12, 13, 14, $15,16,17,18,19,20,21$, and 22), possesses a higher number of endemics than any other state in the country. These endemics belong to four genera: Hechtia (13 spp.), Pitcairnia (1 sp.), Tillandsia (28 spp.), and Viridantha (1 sp.). Of all species present in the state, 95 (55.23\%) are endemic to Mexico and represent eight genera: Bromelia (2 spp.), Catopsis (1 sp.), Greigia (2 spp.), Hechtia (20 spp.), Pitcairnia (6 spp.), Tillandsia (59 spp.), Viridantha (4 spp.) and Werauhia (1 sp.).

It is worth pointing out that the majority of the Bromeliaceae collections are concentrated in the north, north-west and center portions of the state along its principal roadways (Fig. 1). Collections are known from 226 (39.64\%) of the 570 municipalities and from all of the districts (Table 1).

The analysis of species number by altitude (Fig. 2) shows that 79 species, or $45.93 \%$ of the total, ocurr between 1,750 and $2,000 \mathrm{~m}$. The altitudinal average varies between 30 and 45 species in the other altitudinal intervals, and decreases suddenly above $2,500 \mathrm{~m}$. A similar analysis for the collections is showed in figure 3 . The graph shows two peaks, one between 0 and $250 \mathrm{~m}$ and another between 1,750 and 2,000 $\mathrm{m}$. The distribution of the collections between intervals is heterogeneous. 
Table 1. Number of collections and indices of collection density fot each district.

\begin{tabular}{|c|c|c|c|c|}
\hline District & $\begin{array}{l}\text { Number of } \\
\text { collections }\end{array}$ & $\mathrm{Km}^{2}$ & Collection index & $\begin{array}{c}\text { Standarized } \\
\text { collection index }\end{array}$ \\
\hline Juchitán & 193 & 13189 & 1.46 & 24.11 \\
\hline Ixtlán de Juárez & 174 & 2867 & 6.07 & 100.00 \\
\hline Tehuantepec & 135 & 6015 & 2.24 & 36.98 \\
\hline Tuxtepec & 109 & 5515 & 1.98 & 32.57 \\
\hline Cuicatlán & 85 & 2183 & 3.98 & 64.16 \\
\hline Pochutla & 88 & 3738 & 2.35 & 38.79 \\
\hline Tlacolula & 84 & 3097 & 2.71 & 44.69 \\
\hline Teotitlán & 83 & 2305 & 3.60 & 59.33 \\
\hline Tlaxiaco & 63 & 2669 & 2.36 & 38.89 \\
\hline Etla & 54 & 2219 & 2.43 & 40.10 \\
\hline Teposcolula & 57 & 1543 & 3.69 & 60.87 \\
\hline Mixe & 53 & 4922 & 1.08 & 17.74 \\
\hline Huajuapam & 49 & 3270 & 1.50 & 24.69 \\
\hline Miahuatlán & 46 & 3959 & 1.16 & 19.14 \\
\hline Yautepec & 44 & 4259 & 1.03 & 17.02 \\
\hline Villa Alta & 39 & 1162 & 3.36 & 55.30 \\
\hline Juxtlahuaca & 41 & 1859 & 2.21 & 36.34 \\
\hline Coixtlahuaca & 28 & 1688 & 1.66 & 27.33 \\
\hline Putla & 32 & 2553 & 1.25 & 20.65 \\
\hline Nochixtlán & 29 & 2767 & 1.05 & 17.27 \\
\hline Juquila & 28 & 3514 & 0.80 & 13.13 \\
\hline Choapam & 21 & 2725 & 0.77 & 12.70 \\
\hline Centro & 19 & 546 & 3.48 & 57.34 \\
\hline Sola de Vega & 16 & 3635 & 0.44 & 7.25 \\
\hline Silacayoapam & 10 & 1800 & 0.56 & 9.15 \\
\hline Ejutla & 4 & 940 & 0.43 & 7.01 \\
\hline Zaachila & 3 & 545 & 0.55 & 9.07 \\
\hline Jamiltepec & 3 & 4276 & 0.07 & 1.16 \\
\hline Zimatlán & 1 & 975 & 0.10 & 1.69 \\
\hline Ocotlán & 1 & 858 & 0.12 & 1.92 \\
\hline Without data & 43 & - & - & - \\
\hline
\end{tabular}




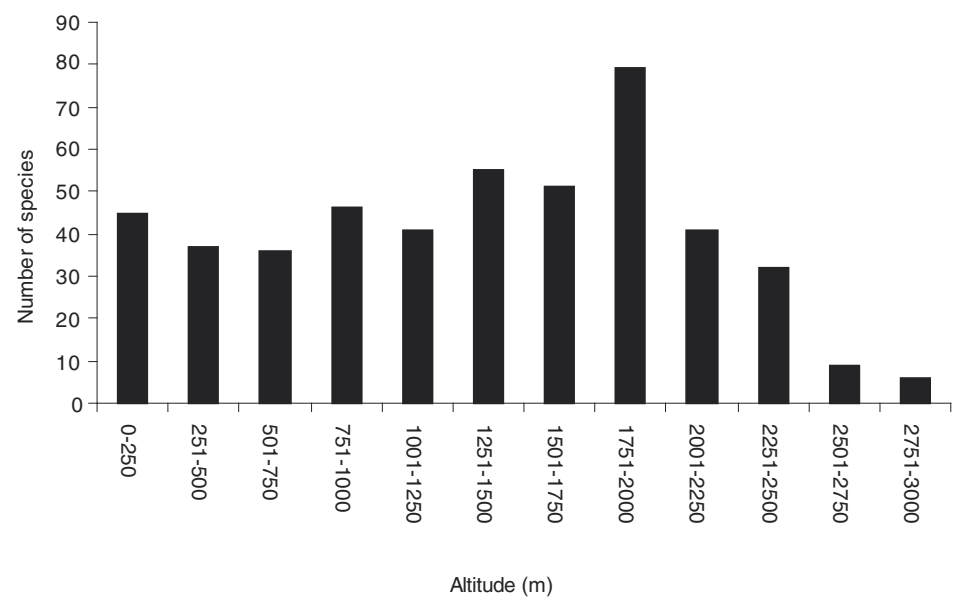

Fig. 2. Altitudinal distribution of the bromeliad species in Oaxaca.

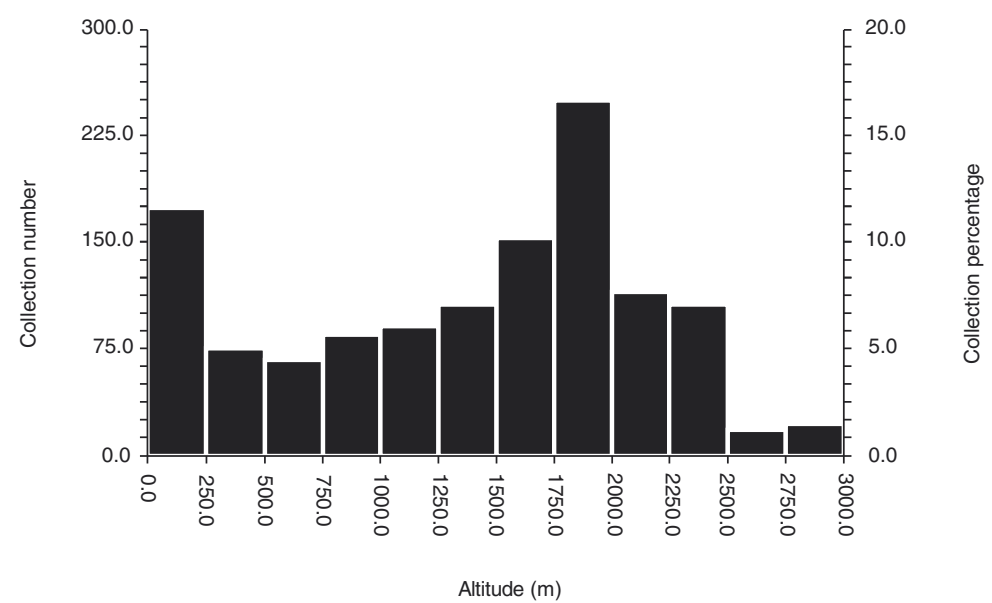

Fig. 3. Altitudinal distribution of the bromeliad collections in Oaxaca. 
Concerning genera (Fig. 4), their distribution is more or less homogeneous, the average varying between 7 and 11 genera per altitudinal interval, and, as with species, they decrease suddenly above 2,500 m. All the Oaxacan species of Aechmea, Billbergia, Bromelia, Fosterella, Guzmania, and Vriesea, grow below 1,500 m. The genus Tillandsia is well represented from the sea level to 3,000 m.

The species accumulation curve (Fig. 5) shows that even though there has been a substantial increase in the number of Bromeliaceae species and collections reported, the asymptote had not been reached. This suggests that the state has not been sufficiently explored. The rarefaction curve, and the Chao and Jacknife estimates show that the predicted number of Bromeliaceae species for the state varies between 219 and 243, and thus between 50 and 74 species remain to be encountered yet.

These results can be explained by the fact that there is a tendency to collect along highways and roads, and this has led to a disproportionate amount of collecting in relatively few areas. In contrast, there are many areas that have not been explored at all. For example, of the 570 municipalities, only in 226 are known collections of Bromeliaceae. In order to increase our knowledge about the floristics and biogeography of the family in the state, it is necessary to undertake a much more extensive sampling in areas that are far from the principal roads. However, it should be noted that a large portion of Oaxaca (and Mexico in general) has suffered severe loss of primary vegetation, and it is therefore possible that even with an extensive collecting effort, few new records may be found.

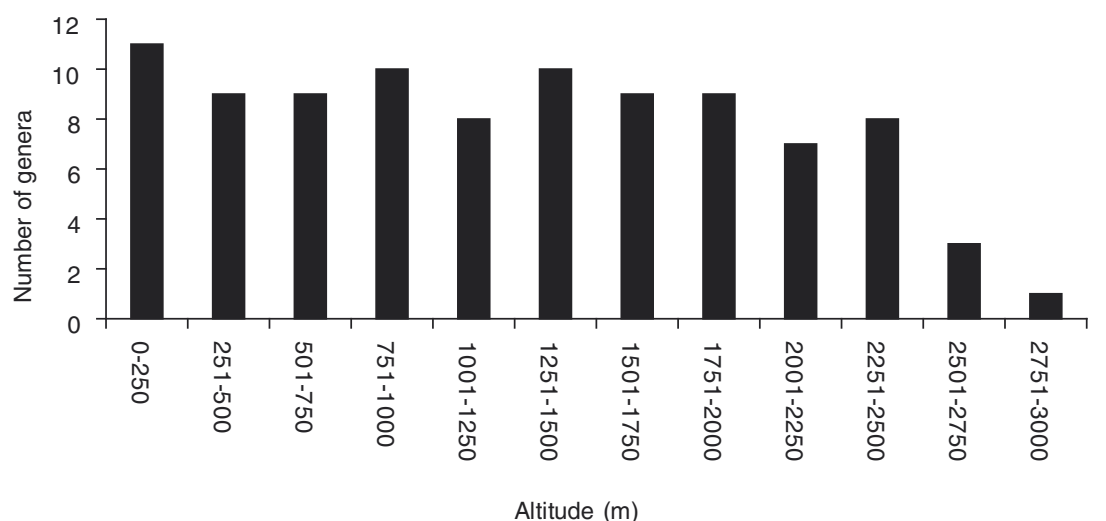

Fig. 4. Altitudinal distribution of the bromeliad genera in Oaxaca. 


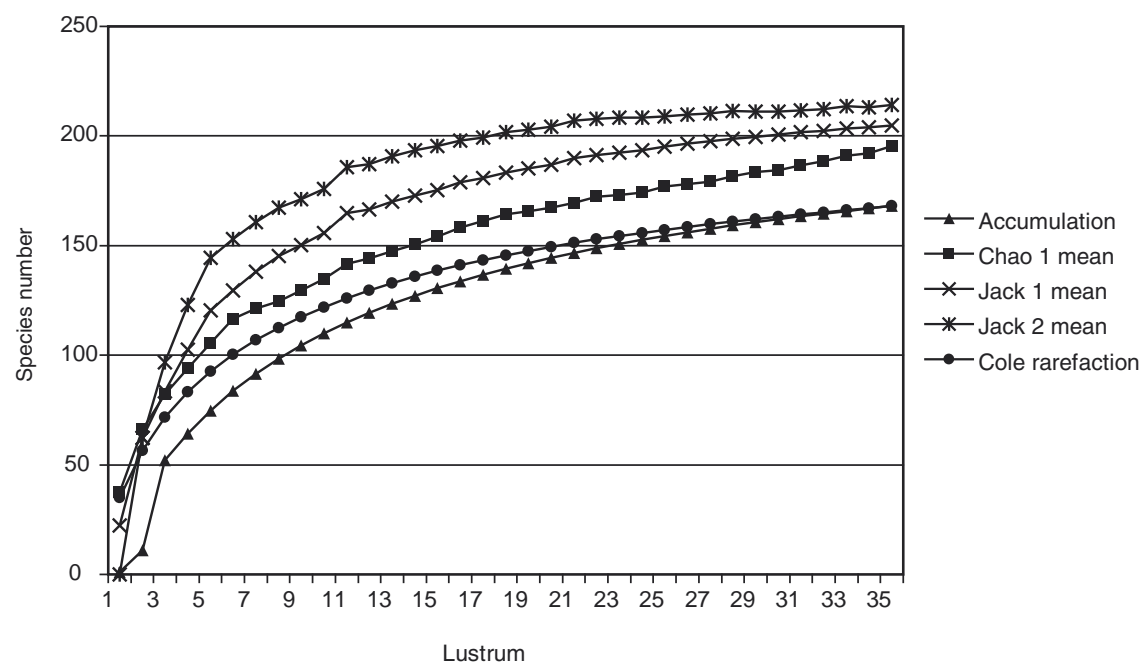

Fig. 5. Rarefaction and species accumulation curves.

With respect to the comparison between the bromeliad flora of Oaxaca with that of the neighbour states, the dendrogram shows that the Floras of Chiapas and Veracruz are very similar and both, as a group, are highly similar to that of Oaxaca (Fig. 6).

The similarity of the Oaxacan bromeliad flora with that of Chiapas-Veracruz is mainly attributed to the Mesoamerican influence in these states. Another factor is the continuous extension of vegetation between these three states, that is represented by tropical forest that extends from northern South America through Central America. The southern states of Mexico represent the northernmost limit of the distribution of various taxa, and in these states the geographic and biological conditions have generated a particular flora. Puebla also shares some elements with the Oaxaca-Chiapas-Veracruz group, but is clearly separate from them (Jaccard index = 0.22). Puebla appears to possess a much stronger influence from the Eje Neovolcánico Transmexicano and the Sierra Madre Oriental. The bromeliad flora of Guerrero has the least similarity in comparison that of the other states analyzed (Jaccard index $=0.21$ ).

Although Oaxaca shares many floristic elements with adjacent regions, it represents a special case, due to its great ecological diversity, ranging from the arid regions of the north to the coastal regions and central zones, the latter of which include cloud forests and a grand extension of coniferous forests (Table 2). 


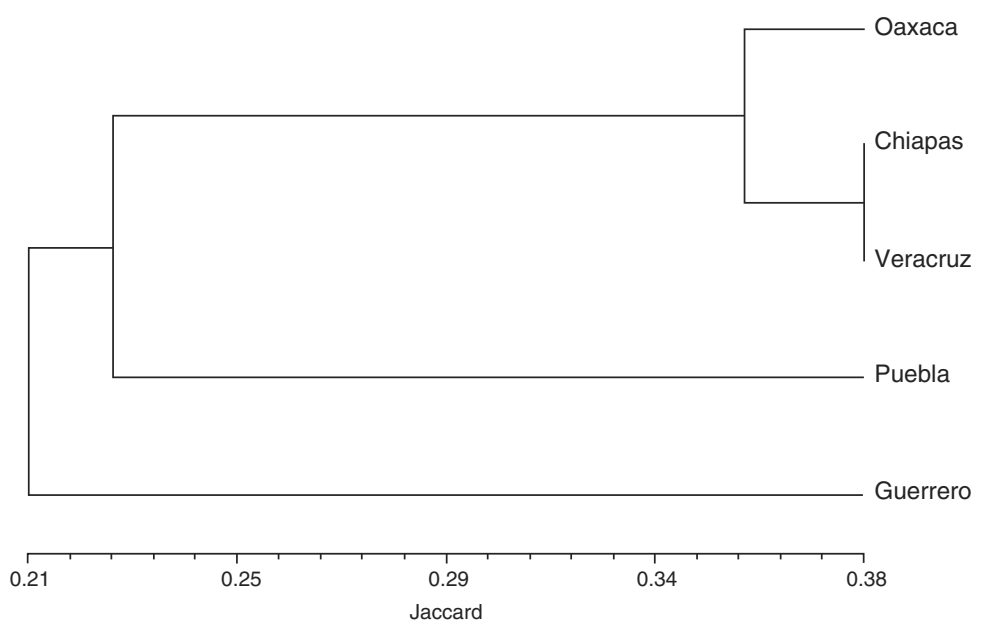

Fig. 6. Similarity of the bromeliad floras between Oaxaca and adjacent states.

With regard to the comparison of bromeliads among the types of vegetation present in the state, the dendrogram (Fig. 7) shows that if we exclude from the analysis the Palmares (because it is generally found distributed in small patches within other types of vegetation where it is associated with humid and generally warm climates or secondary vegetation), there are two main groups (Jaccard Index $=0.04$ ). One contains the majority of the vegetation types (seven in total). Within this graph Bosque de Coníferas and the Bosque de Quercus are the most similar (Jaccard Index $=0.39$ ) and are grouped with the Bosque Mesófilo de Montaña (Jaccard Index $=0.32$ ). The second main group encompasses two subgroups: one conformed by the Vegetación Acuática (mainly Manglares) and the Bosque Espinoso which are always found in the southeast coastal region of the state, and the other represented by the Bosque Tropical Subcaducifolio. Factors such as soil type, temperature and humidity microhabitats and other environmental conditions where these communities are found could be why the bromeliad floras are so similar within these vegetation types.

Of the 11 types of vegetation (sensu Rzedowski, 1978) present in the state, the Bosque de Quercus is the richest in number of species (83), followed by Bosque Tropical Caducifolio with 74 species, and Bosque Mesófilo de Montaña with 73 taxa. Five species are restricted to Bosque de Coníferas: Tillandsia festucoides, $T$. leucolepis T. quaquaflorifera, T. x marceloi, and T. wuelfinghoffi. Thirteen species 
Table 2. Total number of known species by state, shared species with Oaxaca and similarity index (Jaccard).

\begin{tabular}{lccc}
\hline State & $\begin{array}{c}\text { Total number of } \\
\text { known species }\end{array}$ & $\begin{array}{c}\text { Number shared } \\
\text { with Oaxaca }\end{array}$ & Similarity index (Jaccard) \\
\hline Chiapas & 123 & 75 & 34.42 \\
Guerrero & 88 & 55 & 27.5 \\
Puebla & 57 & 44 & 24.44 \\
Veracruz & 89 & 69 & 36.9 \\
\hline
\end{tabular}

are known only from Bosque de Encino: Bromelia hemisphaerica, Hechtia nuusviorum and two undescribed species of Hechtia, Pitcairnia schiedeana, P. tuerckheimii, Tillandsia atroviolacea, T. lampropoda, T. rettigiana, T. rubrispica, T. ulrici, T. yerbasantae, and Viridantha penascoenesis. Thirteen species are exclusive to Bosque Mesófilo de Montaña: Catopsis nitida, C. oerstediana, Greigia vanhyningi, Racinaea adscendens, Tillandsia belloensis, T. copalaensis, T. kirchhoffiana, T. kolbii, T. laui, T. lautneri, T. leiboldiana, T. mixtecorum, and Werauhia nocturna. Seventeen species are confined to Bosque Tropical Caducifolio: Hechtia caudata, $H$. fosteriana, $H$. fragilis, $H$. lanata, $H$. lyman-smithii, $H$. marnier-lapostollei, and three additional undescribed species, Tillandsia atenangoensis, T. califanii, T. glabrior, T. ortgiesiana, T. pseudobaileyi, T. schatzlii, T. schusteri, and T. vernardoi. Twelve species are limited to Bosque Tropical Perennifolio: Aechmea magdalenae, A. nudicaulis, Catopsis wawranea, Pitcairnia undulata, Tillandsia flavobracteata, T. foliosa, T. limbata, T. pruinosa, Vriesea heliconioides, V. malzinei, Werauhia gladioliflora, and W. nutans. Tillandsia socialis grows only in Bosque Tropical Subcaducifolio. Two species are restricted to Matorral Xerófilo: Hechtia confusa, and Viridantha mauryana. Tillandsia balbisiana occurs solely in Bosque Espinoso.

\section{ACKNOWLEDGMENTS}

We thank the following herbaria for providing specimens and data used in this work: B, BM, BR, C, CHAP, CICY, CIIDIR, CODAGEM, ECON, ENCB, F, FCME, FI, GH, GOET, HEID, IBUG, IEB, K, LG, LL, M, MA, MEXU, MICH, MO, NY, OXF, P, SEL, SERO, TEX, UAMIZ, UC, UNICACH, US, VT, WU, XAL, and Z; the Elizabeth Bascom Fellowship for Latinoamerican Women and the Mis- 


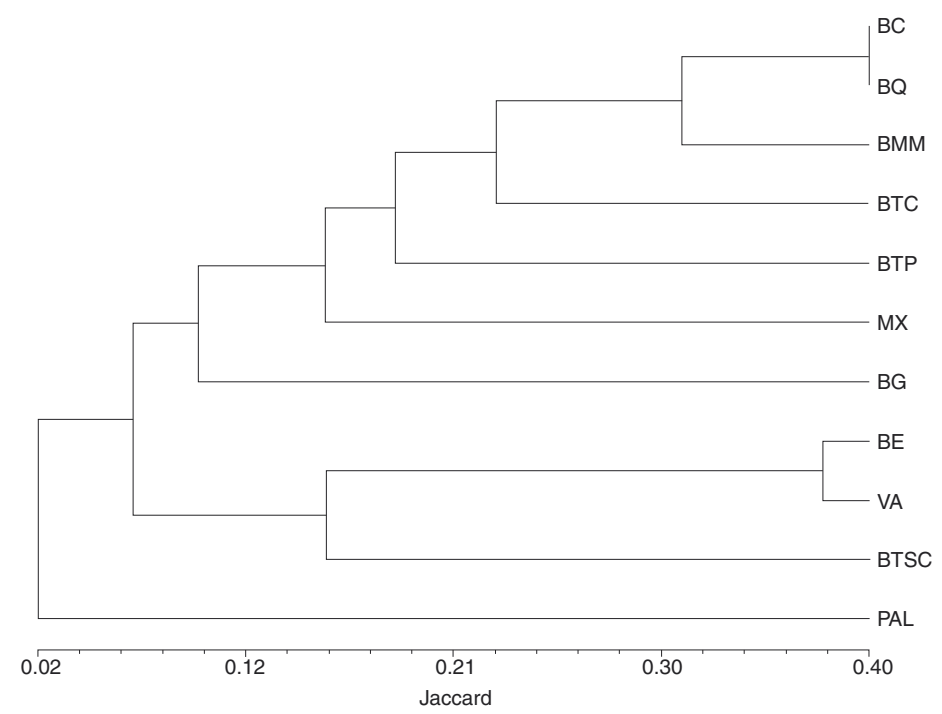

Fig. 7. Similarity of the bromeliad flora between the types of vegetation in Oaxaca. BC: Bosque de Coníferas (Coniferous forest); BQ: Bosque de Quercus (Oak forest); BMM: Bosque Mesófilo de Montaña (Cloud forest); BTC: Bosque Tropical Caducifolio (Tropical deciduous forest); BTP: Bosque tropical perennifolio (Tropical evergreen forest); MX: Matorral xerófilo (Xerophytic scrub); BG: Bosque de Galería (Gallery forest); BE: Bosque Espinoso (Thorn forest); VA: Vegetación Acuática (Aquatic vegetation); BTSC: Bosque tropical subcaducifolio (tropical subdeciduous forest); PAL: Palmar (Palm savanna).

souri Botanical Garden for a scholarship granted to the second author; Jacqueline Ceja Romero and Aniceto Mendoza Ruiz for their invaluable and constant help in the field work; Victor Steinmann for his help with the English translation; and Javier García-Cruz suggestions and improvements to the manuscript.

\section{LITERATURE CITED}

Anonymous. 2003. ESRI. ArcView Ver. 8.1.

Anonymous. 2004. Síntesis de Información Geográfica del Estado de Oaxaca. Instituto Nacional de Estadística Geografía e Informática. Aguascalientes. 166 pp.

Arellano Mijangos, J. J. 2002. Las Bromeliaceae del Estado de Oaxaca: Riqueza florística y potencial ornamental. Tesis profesional. Universidad Autónoma Chapingo. Centro Universitario del Sureste. San José Puyacatengo, Tabasco. 135 pp. 
Baker, J. G. 1889. Handbook of the Bromeliaceae. George Bell \& Sons. London. 243 pp.

Chao, A. 1984. Nonparametric estimation of the numbers of classes in a population. Scandinavian J. Stat. 11: 265-270.

Colwell, R. K. 2004. Estimates: statitistical estimation of species richness and shared species from samples. Version 7. User's guide and application. 2006 (http://viceroy. eeb.uconn.edu/estimates).

Colwell, R. K. \& J. A. Coddington. 1994. Estimating terrestrial biodiversity through extrapolation. Philos. Trans., Ser. B. 345: 101-188.

Espejo, A., A. R. López-Ferrari, I. Ramírez-Morillo, B. K. Holst, H. E. Luther \& W. Till. 2004. Checklist of Mexican Bromeliaceae with notes on species distribution and levels of endemism. Selbyana 25(1): 33-86.

Espejo, A., A. R. López-Ferrari \& I. Ramírez-Morillo. 2005. Bromeliaceae. Flora de Veracruz 136. Instituto de Ecología, A. C. Xalapa, Veracruz. 307 pp.

Grant, J. 1995a. Bromelienstudien. The resurrection of Alcantarea and Werauhia, a new genus. Trop. Subtrop. Pflanzenw. 91: 1-57.

Grant, J. 1995b. Addendum to The resurrection of Alcantarea and Werauhia, a new genus (Bromeliaceae; Tillandsioideae). Phytologia 78(2): 119-123.

Luther, H. E. 2006. An alphabetical list of bromeliad binomials. Bromeliad Society International. Sarasota. 119 pp.

McVaugh, R. 1989. Bromeliaceae. In: Anderson, W. R. (ed.). Flora Novo-Galiciana. The University of Michigan Herbarium. Ann Arbor. pp. 4-79.

Mez, C. 1896. Bromeliaceae. In: De Candolle, C. Monographiae Phanerogamarum 9: 1990.

Ortiz Pérez, M. A., J. R. Hernández Santana \& J. M. Figueroa Mah-Eng. 2004. Reconocimiento fisiográfico y geomorfológico. In: García-Mendoza, A. J., M. J. Ordóñez \& M. Briones-Salas (eds.). Biodiversidad de Oaxaca. Instituto de Biología, Universidad Nacional Autónoma de México, Fondo Oaxaqueño para la Conservación de la Naturaleza, World Wildlife Fund. México, D.F. pp. 43-54.

Palací, C. A. 1997. A systematic revision of the genus Catopsis (Bromeliaceae). Ph. D. Thesis. University of Wyoming. Laramie, Wyoming. 245 pp.

Pulido-Esparza, V. A., A. R. López-Ferrari \& A. Espejo. 2004. Flora bromeliológica del Estado de Guerrero, México: riqueza y distribución. Bol. Soc. Bot. Méx. 75: 55-104.

Ramírez-Morillo, I., G. Carnevali Fernández-Concha \& F. Chi May. 2004. Guía ilustrada de las Bromeliaceae de la porción mexicana de la Península de Yucatán. Centro de Investigaciones Científicas de Yucatán, A. C. Mérida, Yucatán. 124 pp.

Rohlf, F. J. 1998. NTSYSpc. Numerical taxonomy and multivariate analysis system. Version 2. User guide. University of New York. Stony Brook, New York.

Rzedowski, J. 1978. Vegetación de México. Limusa. México, D.F. 432 pp.

Smith, L. B. 1961. Notes on Bromeliaceae, XVII. Phytologia 8(1): 1-13.

Smith, L. B. \& R. J. Downs. 1974. Pitcairnioideae (Bromeliaceae), Flora Neotropica 14(1): 1-658.

Smith, L. B. \& R. J. Downs. 1977. Tillandsioideae (Bromeliaceae), Flora Neotropica 14(2): 659-1492. 
Smith, L. B. \& R. J. Downs. 1979. Bromelioideae (Bromeliaceae), Flora Neotropica 14(3): 1493-2142.

Till, W. 1990. Altbekannt - und trotzdem neu: Tillandsia fuchsii, spec. nov. Bromelie 1990(2): 30-33.

Till, W. 1992. A well-known new species: Tillandsia fuchsii. J. Bromeliad Soc. 42(5): 99102.

Trejo, I. 2004. Clima. In: García-Mendoza, A. J., M. J. Ordóñez \& M. Briones-Salas (eds.). Biodiversidad de Oaxaca. Instituto de Biología, Universidad Nacional Autónoma de México, Fondo Oaxaqueño para la Conservación de la Naturaleza, World Wildlife Fund. México, D.F. pp. 67-85.

Utley, J. \& K. Burt-Utley. 1994. Bromeliaceae. In: Davidse, G., M. Sousa Sánchez \& A. O. Chater (eds.). Alismataceae a Cyperaceae. Universidad Nacional Autónoma de México. Instituto de Biología, Missouri Botanical Garden, The Natural History Museum (London). México, D.F. pp. 89-156.

Victoria, A. 2001. Bromeliaceae. In: Rzedowski, G. C. de, J. Rzedowski y colaboradores. Flora Fanerogámica del Valle de México. 2a. ed. Instituto de Ecología, A.C. y Comisión Nacional para el Conocimiento y Uso de la Biodiversidad. Pátzcuaro, Michoacán. pp. 1179-1187.

Recibido en julio de 2006.

Aceptado en julio de 2007. 


\title{
APPENDIX
}

\author{
Reference List of the Oaxacan Bromeliaceae
}

\section{AechmeA}

1 Aechmea bracteata (Sw.) Griseb., Fl. Brit. W.I. 592. 1864.

Fig.8

Specimens examined: District Juchitán, San Juan Guichicovi, S. Trott, P. Case, D. Dunn, D. Thurm \& C. Dziekanowski 255 (MO); Santa María Chimalapa, R. Torres C. \& C. Martínez 6029 (MEXU). District Putla, Putla Villa de Guerrero, A. Espejo, A. R. López-Ferrari, J. Ceja \& A. Mendoza R. 6794 (UAMIZ(x3)), A. García-Mendoza \& R. Torres C. 1511 (MEXU). District Tuxtepec: Acatlán de Pérez-Figueroa, L. Cortés A. \& R. Torres C. 468 (MEXU(x3)), R. E. Gereau, M. Sousa S., R. Torres C. \& L. Cortés A. 2193 (MEXU(x3), MICH(x2), TEX); Loma Bonita, E. Hernández Xolocotzi 607 (LL(x2)); San José Chiltepec, A. R. López-Ferrari, A. Espejo, J. Ceja, A. Mendoza R., B. Pérez G. \& I. Reyes J. 2105 (IEB(x3) UAMIZ(x3)); R. E. Schultes \& B. P. Reko 550A(GH); San Juan Bautista Tuxtepec, J. Chavelas P. \& L. A. Pérez Jiménez 85 (MEXU), A. García-Mendoza 4470 (MEXU(x2)), F. Miranda 4134 (MEXU).

2 Aechmea lueddemanniana (K. Koch) Mez, in Engl., Pflanzenr. IV. 32 (Heft 100, 1): 120. 27-II-1934.

Specimens examined: District Juchitán, Santa María Chimalapa, J. Rivera H., S. Salas M., R. García S., Y. Arellanes C. \& A. Sánchez A. 689 (SERO); J. Rivera H., E. Martínez S. \& C. Perret 1232 (MEXU). District Teotitlán, San Bartolomé Ayautla, $R$. Torres C., $R$. Cedillo T. \& L. Cortés A. 853 (MEXU). District Tuxtepec San José Chiltepec, R. E. Schultes \& B. P. Reko 550B (GH). District Villa Alta, San Ildefonso Villa Alta: H. Galeotti 4918 (BR (x2)).

3 Aechmea magdalenae (André) André ex Baker, Handb. Bromel. 65-66. 1889. Fig. 8 Specimens examined: District Choapam, San Juan Lalana, R. E. Schultes \& B. P. Reko $795 a$ (ECON). District Tuxtepec, San Felipe Usila, J. I. Calzada \& M. Vartas 15230 (MEXU); San Juan Bautista Tuxtepec, F. Miranda 4224 (MEXU).

4 Aechmea mexicana Baker, J. Bot. 17: 165. 1879.

Fig. 8 Specimens examined: District Choapam, Santiago Yaveo, A. García-Mendoza, $R$. Torres C. \& L. Cortés A. 2917 (MEXU). District Pochutla, San Pedro Pochutla, C. Conzatti, B. P. Reko \& Makrinius 3137 (MEXU). District Tuxtepec, San José Chiltepec G. Martínez C. 563 (GH); Santa María Jacatepec, R. Torres C. \& E. Martínez S. 10985 (IEB, MEXU), R. Torres C. \& L. Cortés A. 11457 (MEXU), R. Torres C. 12160 (MEXU). Without precise locality, J. Rivera H. \& S. Escobedo 75 (UAMIZ).

5 Aechmea nudicaulis (L.) Griseb., Fl. Brit. W.I. 593. 1864.

Fig. 9 Specimens examined: District Tuxtepec, San José Chiltepec, G. Martínez C. 83 (GH). 
6 Aechmea tillandsioides (Mart. ex Schult. \& Schult. f.) Baker, J. Bot. 17: 134. 1879.

Fig. 8

Specimens examined: District Choapam, Santiago Jocotepec, P. Reko 4055 (US). District Juchitán, Santa María Chimalapa, H. Hernández G. 1271 (UAMIZ), J. Rivera H., R. de Santiago G., T. Álvarez R. \& D. Acuca V. 583 (UAMIZ), J. Rivera H., E. Martínez S. \& C. Perret 1400 (MEXU). District Tuxtepec, San José Chiltepec, G. Martínez C. 139 (GH); San Juan Bautista Valle Nacional, R. E. Schultes \& B. P. Reko 619 (GH(x2)).

\section{BillBergia}

1 Billbergia pallidiflora Liebm., Ann. Sci. Nat. Bot. sér. 4. 2: 373. 1854.

Fig. 8 Specimens examined: District Juchitán, Asunción Ixtaltepec, J. Meave del C. \& E. Pérez G. 1867 (MEXU); Ciudad Ixtepec, C. Martínez R. 1113 (MEXU); El Barrio de la Soledad, R. Torres C., L. Cortés A. \& M. L. Torres C. 9737 (IEB(x3), MEXU); Santo Domingo Petapa, E. W. Nelson 2647 ([S. \& D., 1979] US). District Pochutla, San Miguel del Puerto, J. F. Castrejón R., E. Martínez S. \& M. Elorsa C. 970 (MEXU), E. Martínez S., M. Elorsa C. \& C. Perret 32054 (MEXU), J. Rivera H. 865 (UAMIZ) A. Saynes V. \& M. Elorsa 3119 (UAMIZ), A. Saynes V., M. Elorsa C., L. Schibli \& V. Chao 2353 (MEXU); Santa María Huatulco, G. Castillo C., P. Zamora C. \& A. González 9717 (XAL). District Tehuantepec, Magdalena Tequisistlán, E. Matuda 37662 (ENCB, MEXU), O. van Hyning 541 ([S. \& D., 1979] US); Santiago Lachiguiri, A. Campos V. \& R. Torres C. 3624 (MEXU); Santo Domingo Tehuantepec, M. L. Torres C., R. Torres C., C. Martínez \& J. Martínez 864 (MEXU), R. Torres C. 4288 (MEXU), R. Torres C. \& J. L. Villaseñor 5138 (MEXU), R. Torres C. \& C. Martínez 8332 (MEXU(x2)). District Yautepec, San Carlos Yautepec, E. Matuda \& Cols. s. n. (MEXU, MO).

\section{BROMELIA}

1 Bromelia hemisphaerica Lam., Encycl. 1: 145. 1783.

Fig. 8 Specimens examined: District Centro, Oaxaca de Juárez, C. G. Pringle 5861 (GH), without municipio indicated, C., H. \& C. Conzatti 1171 (GH, MEXU), C. Conzatti 3624 (MEXU(x2)). District Zimatlán, Zimatlán de Álvarez, C. Conzatti 5291 (GH, MEXU, $\mathrm{MICH})$.

2 Bromelia karatas L., Sp. Pl. 285. 1753.

Fig. 8

Specimens examined: District Pochutla, San Miguel del Puerto, M. Elorsa C. 2152 (MEXU), F. López 78 (MEXU) S. Salas M., A. Sánchez \& A. de Ávila 4928 (MEXU), A. Saynes V., M. Elorsa C. \& N. Velázquez 2169 (MEXU); Santa María Huatulco, G. Castillo C., P. Zamora C. \& F. González 9592 (MEXU, XAL), C. Conzatti, B. P. Reko \& Makrinius 3237 (MEXU), B. P. Reko 3237 (US), A. Saynes V. \& A. Sánchez 5181 (MEXU). District Putla, Putla Villa de Guerrero, E. Solano C. 381 (TEX(x2)). District Yautepec, San Carlos Yautepec, E. Martínez S., M. Elorsa C. \& C. Perret 32097 (MEXU, UAMIZ); San Juan Lajarcia, R. Torres C., L. Cortés A. \& C. Martínez 9856 (IEB, MEXU). 
3 Bromelia palmeri Mez, in C. DC., Monogr. Phan. 9: 40. 1896.

Specimens examined: District Juchitán, Asunción Ixtaltepec, C. Gallardo H., E. A. Pérez García, B. Reyes, J. Meave \& J. Reyes 1488 (MEXU), C. Gallardo H., E. Pérez García, I. Sánchez G., L. Lozada \& B. Reyes 1513 (MEXU(x2)), S. Salas M. 4565 (MEXU); Ciudad Ixtepec, $R$. Merrill King 1673 (TEX), R. Merrill King 1713 (TEX, US). District JuquiLA, San Pedro Tututepec, G. Morales s. n. (FCME). District Pochutla, Santa María Huatulco, R. Cedillo T., R. Torres C. \& L. Cortés A. 1699 (IEB, XAL), A. Reyes-García \& G. Ibarra 2683 (IEB, MEXU), C. Tovillas H. 433 (MEXU). District TehuantePec, San Pedro Huamelula, M. Elorsa C. 2319 (MEXU, SERO), J. Rivera H., S. Salas M. \& E. Martínez S. 1697 (MEXU, SERO); Santiago Astata, S. Salas M. 3177 (MEXU); Santo Domingo Tehuantepec, M. L. Torres C., R. Torres C. \& C. Martínez 426 (MEXU(x2)), R. Torres C. \& C. Martínez 5567 (IEB, MEXU).

4 Bromelia pinguin L., Sp. Pl. 285. 1753. Fig. 8 Specimens examined: District Juchitán, Asunción Ixtaltepec, J. Chavelas P. \& C. Zamora S. ES-4824 (MEXU); San Francisco del Mar, S. Salas M. 1070 (UAMIZ); San Juan Guichicovi, N. Antonio B. \& M. Heinrich GUI 146 (MEXU); Santiago Niltepec, R. Merrill King 1844 (TEX). District Pochutla, San Miguel del Puerto, M. Cerón C., S. Salas M., E. Torres B. \& R. García S. 381 (MEXU, SERO, UAMIZ), M. Elorsa C., L. Schibli \& E. Gamboa 4761 (MEXU), S. Salas M., J. Rivera H., R. García S., J. Meave del C. \& M. Elorsa 2057 (SERO); Santa María Huatulco, G. Castillo C., P. Zamora C. \& A. González 9240 (IEB, MEXU, XAL), G. Castillo C., P. Zamora C. \& A. González 9294 (XAL), R. Torres C. \& R. Cedillo T. 890 (MEXU, MO); Santa María Tonameca, B. Hansen, J. Hansen \& M. Nee 1533 (MEXU, US(x3)). District Tehuantepec, San Pedro Huamelula, M. Elorsa C., L. Schibli \& E. Gamboa 4762 (MEXU), J. Rivera H., E. Torres B., H. Morales I. \& A. de Ávila H. 413 (UAMIZ), J. Rivera H., S. Salas M. \& A. Sánchez A. 738 (SERO, UAMIZ), A. Saynes V., M. Elorsa C., S. Salas M. \& E. Gamboa 2666 (MEXU); Santo Domingo Tehuantepec, C. Martínez R. 592 (MEXU), R. Merrill King 241 (MEXU, MICH, TEX), R. Merrill King 1169 (MICH, TEX, US), Preuss s. n.([S. \& D., 1979] B, like B. alsodes), M. L. Torres C., $R$. Torres C. \& C. Martínez 369 (MEXU), R. Torres C. \& C. Martínez 4890 (MEXU). District Tlacolula, San Pablo Villa de Mitla, J. N. Rose \& J. S. Rose 11293 (US).

4 CATOpsis

1 Catopsis berteroniana (Schult. \& Schult. f.) Mez, in C. DC., Monogr. Phan. 9: 621. 1896.

Fig. 9

Specimens examined: District Juchitán, Asunción Ixtaltepec, J. Ceja, A. Mendoza R., R. Cerros T. \& V. Steinmann 386 (UAMIZ(x2)), A. Espejo, A. R. López-Ferrari, J. Ceja, A. Mendoza R. \& I. Ramírez M. 6485 BIS (UAMIZ), R. Torres C. \& C. Martínez 6086 (MEXU), R. Torres C. \& E. Martínez S. 11233 (IEB, MEXU); El Barrio de la Soledad, A. Espejo, A. R. López-Ferrari, J. Ceja, A. Mendoza R. \& I. Ramírez M. 6475 (UAMIZ(x2)), R. Fernández N. 4194 (ENCB), S. Zamudio R. 6349 (IEB); Santa María Chimalapa, H. Hernández G. 368 (UAMIZ). District Mixe, Totontepec Villa de Morelos, J. Rivera R. \& G. J. Martin 1092 (MEXU). District Pochutla, San Pedro Pochutla, J. Rzedowski 21210 A (ENCB, 
Espejo-Serna et al.: Bromeliad flora of Oaxaca

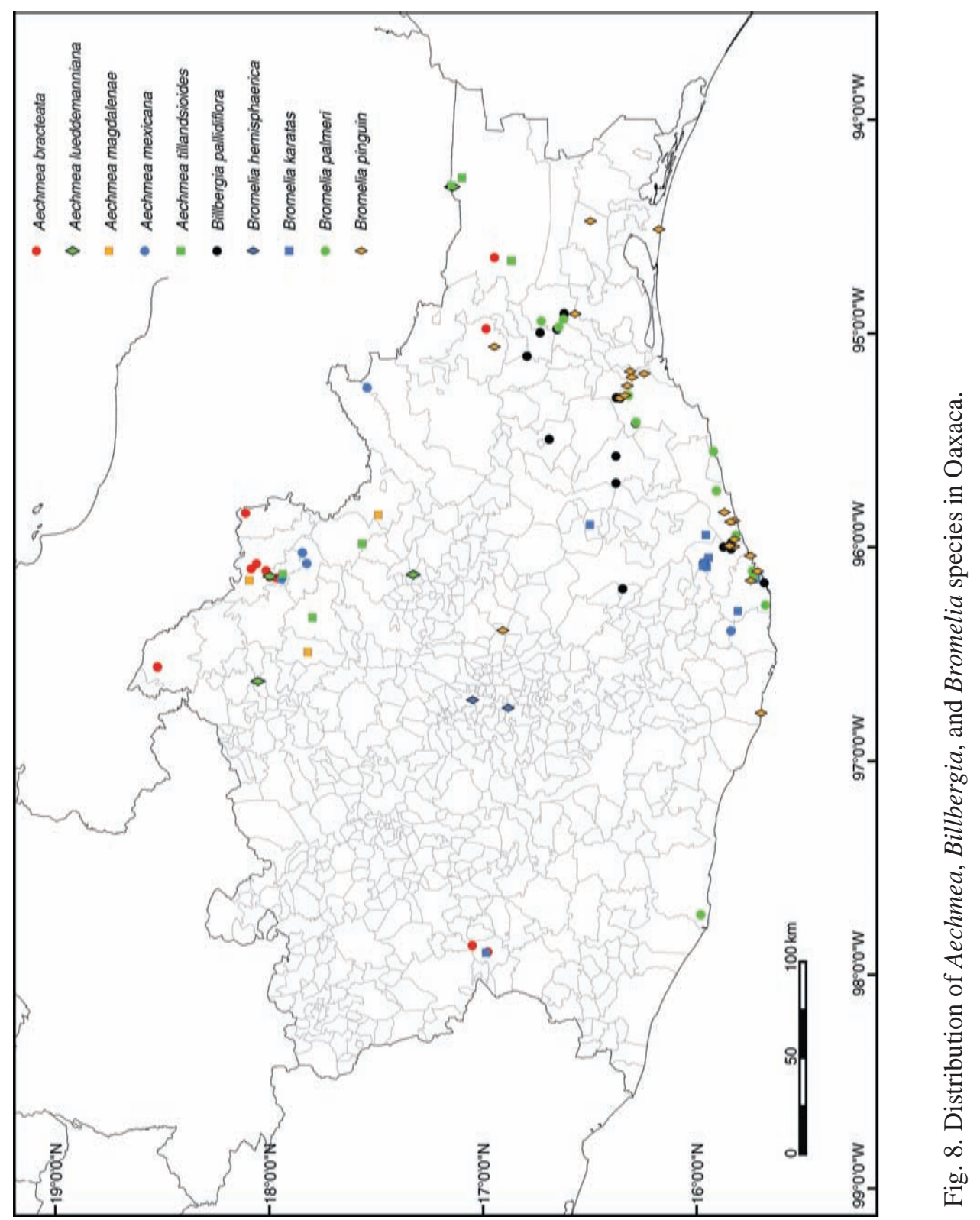


MEXU(x2), MiCH, TEX). District Tehuantepec, Guevea de Humboldt, A. Campos V. 3880 (MEXU(x2)), A. Campos V. 4192 (MEXU), R. Torres C. \& L. Cortés A. 8895 (IEB, MEXU).

2 Catopsis compacta Mez, Bull. Herb. Boissier sér. 2. 3: 140. 1903.

Specimens examined: District Centro, Oaxaca de Juárez, C. Conzatti \& V. González 1172 (GH). District Cuicatlán, San Pedro Jaltepetongo, R. Medina L., L. Alvarado C. \& J. Sandoval 1016a (MEXU(x2)); Santiago Nacaltepec, A. Salinas T., E. Martínez C. \& R. Martínez S. 6583 (MEXU), A. Salinas T., E. Martínez C. \& R. Martínez S. 6819 (MEXU), L. C. Smith 543 (B, GH). District Ejutla, Ejutla de Crespo, J. Calónico S. 26675 (UAMIZ). District EtLA, San Jerónimo Sosola, B. Leuenberger \& C. Schiers 2757 (MEXU); San Pablo Etla, A. R. López-Ferrari, A. Espejo, J. García-Cruz, E. Yáñez G. \& S. Acosta C. 1857 (UAMIZ(x2)). District Huajuapam, San Andrés Dinicuiti, W. Boege 3020 (MEXU). District Ixtlán, San Miguel Yotao, X. Munn, A. Blanco, R. del Castillo, R. Rivera \& N. Sánchez 342 (MEXU, XAL). District Juquila, San Juan Lachao, A. R. López-Ferrari, A. Espejo, J. García-Cruz, E. Yáñez G. \& S. Acosta C. 1885 (UAMIZ). District JuxtlahuAcA, San Juan Mixtepec, J. Reyes S. 112 (MEXU), J. Reyes S. 2016 (MEXU); Santiago Juxtlahuaca, J. I. Calzada 20964 (MEXU). District Nochixtlán, Santiago Huauclilla, A. Salinas T., E. Martínez C. \& R. Martínez S. 6824 (MEXU). District Putla, Putla Villa de Guerrero, A. Espejo, A. R. López-Ferrari, J. Ceja \& A. Mendoza R. 6789 (UAMIZ(x2)), A. García-Mendoza, R. Torres C. \& L. Cortés A. 3123 (MEXU), S. Zona, O. Dorado \& R. Torres C. 233 (MEXU(x2)). District Sola de Vega, Villa Sola de Vega, G. Davidse \& J. Davidse 9651 (MO). District Teotitlán, Santa María Ixcatlán, P. Tenorio L. 18351 (MEXU). District Tlacolula, San Lorenzo Albarradas, A. Espejo \& A. R. López-Ferrari 3926 (UAMIZ); Santo Domingo Albarradas, J. Ceja, A. Espejo, A. R. López-Ferrari \& A. Mendoza R. 1581 (UAMIZ), J. Ceja, A. Espejo, A. R. López-Ferrari \& A. Mendoza R. 1582 (UAMIZ), J. Ceja, A. Espejo, A. R. López-Ferrari \& A. Mendoza R. 1595 (UAMIZ(x3)). District Tlaxiaco, Santiago Yosondúa, J. Ceja, A. Espejo \& A. Mendoza R. 1118 (UAMIZ), J. Ceja, A. Espejo, A. R. López-Ferrari \& A. Mendoza R. 1124 (UAMIZ), J. Ceja, A. Espejo, A. R. López-Ferrari, A. Mendoza R. \& G. Carnevali 1754 (UAMIZ(x2). District YautePec, Nejapa de Madero, A. Salinas T. \& E. Martínez C. 81909-B (MEXU).

3 Catopsis floribunda (Brongn.) L. B. Sm., Contr. Gray Herb. 117: 5. $1937 . \quad$ Fig. 9 Specimens examined: District Juchitán, Asunción Ixtaltepec, T. MacDougall s. n. ([S. \& D., 1979] US). Without precise locality, T. MacDougall s. n. (US). District ChoApAm, Santiago Yaveo, R. E. Schultes $561(\mathrm{~K})$.

4 Catopsis morreniana Mez, in C. DC., Monogr. Phan. 9: 628-629. 1896.

Fig. 9

Specimens examined: District Juchitán, Asunción Ixtaltepec, E. A. Pérez-García \& B. Reyes R. 1263 (MEXU); Santa María Chimalapa, H. Hernández G. 261 (UAMIZ), H. Hernández G. 759 (UAMIZ). District Tuxtepec, San Felipe Usila, R. E. Schultes \& B. P. Reko 672a (GH); San José Chiltepec, G. Martínez C. 136 (GH, LL, MEXU); San Juan Bautista Tuxtepec, J. I. Calzada 14424 (UAMIZ); Santa María Jacatepec, R. Torres C. \& L. Cortés A. 11513 (MEXU). 
5 Catopsis nitida (Hook.) Griseb., Fl. Brit. W.I. 599. 1864.

Fig. 10

SPecimens eXAmined: District IXtLÁN, Santiago Comaltepec, O. van Hyning s. n. ([S. \& D., 1979] US).

6 Catopsis nutans (Sw.) Griseb., Fl. Brit. W.I. 599. 1864.

Fig. 9

Specimens examined: District Ixtlán, Ixtlán de Juárez, R. García S. 777 (MEXU). District Putla, Santa María Ipalapa, A. Espejo, A. R. López-Ferrari, J. Ceja \& A. Mendoza R. 6797 (UAMIZ), A. Espejo, A. R. López-Ferrari, J. Ceja \& A. Mendoza R. 6798 (UAMIZ). District Tuxtepec, San Juan Bautista Valle Nacional, D. Thurm, D. Dunn, S. Trott, P. Case \& C. Dziekanowski 219 (ENCB), D. Thurm, D. Dunn, S. Trott, P. Case \& C. Dziekanowski 220 (MO); San Pedro Ixcatlán, J. I. Calzada 10266 (XAL); Santa María Jacatepec, A. $R$. López-Ferrari, A. Espejo \& G. Carnevali 3190bis (UAMIZ), A. R. López-Ferrari, A. Espejo \& G. Carnevali 3193 (UAMIZ), J. I. Calzada 14458 (UAMIZ). District Villa Alta, San Miguel Talea de Castro, J. Ceja, A. Mendoza R., A. Espejo \& A. R. López-Ferrari 1604 (UAMIZ), F. Liebmann s. n. (Monogr. Phan. 9: 631).

7 Catopsis oerstediana Mez, in C.DC Monogr. Phan. 9: 630. 1896.

Fig. 9 Specimens eXamined: District Juchitán, San Miguel Chimalapa, M. Ishiki I. 1265 (MEXU, MO), M. Ishiki I. 1413 (MEXU), M. Ishiki I. 1678 (MEXU).

8 Catopsis paniculata E. Morren, in Jacob-Makoy, Cat. Hort. no. 121: 1. X-1883. Fig. 9 Specimens examined: District Cuicatlán, San Juan Bautista Cuicatlán, C. Conzatti 3870 (MEXU), H. \& C. Conzatti \& Y. Cancino G. 2375 (MEXU). District Choapam, Santiago Choapam, F. Liebmann 42 ([S. \& D., 1979] C, like C. hahnii). District IxtLÁN, Ixtlán de Juárez, D. H. Lorence, A. García-Mendoza \& R. Cedillo T. 3588 (MEXU), Y. Arellanes C., E. Torres B. \& J. González C. 408 (MEXU); Santiago Xiacuí, D. H. Lorence, A. GarcíaMendoza, G. Martin \& R. Cedillo T. 3639 (MEXU, MO). District Mixe, Totontepec Villa de Morelos, E. Ramírez G. \& P. Ramírez C. 388 (IEB, MO, XAL), J. Rivera R. JR0776 (MEXU). District Teotitlán, San Bartolomé Ayautla, A. Flores M. 1536 (UAMIZ); San Lucas Zoquiapam, R. E. Schultes 822 (GH); Teotitlán de Flores Magón, P. Tenorio L. \& L. Kelly 20144 (MEXU). District Villa Alta, San Miguel Talea de Castro, H. Galeotti 4919 (BR).

9 Catopsis sessiliflora (Ruiz \& Pav.) Mez, in C. DC., Monogr. Phan. 9: 625. 1896. Fig. 9 Specimens examined: District Ixtlán, Ixtlán de Juárez, E. Martínez S., O. Téllez, G. Davidse \& J. Davidse 8738 (MEXU), J. C. Flores V., I. Sánchez G. \& J. Rivera H. 93 (UAMIZ); San Juan Evangelista Analco, O. van Hyning 5968 (US); Santiago Comaltepec, $A$. Espejo, A. R. López - Ferrari, J. Ceja, A. Mendoza R. \& G. Carnevali 6693 (UAMIZ), W. L. Graham 1417 (MICH), G. Pérez Pablo \& D. Orona 139 (MEXU). Disctrict Juchitán, San Miguel Chimalapa, S. Maya J. 1825 (MEXU); Santa María Chimalapa, J. Rivera H., R. de Santiago G., T. Álvarez R. \& D. Acuca V. 610 (UAMIZ). District Miahuatlán, San Jerónimo Coatlán, A. Campos V. \& J. Reyes 1319 (MEXU), A. Campos V. \& L. Cortés A. 2230 (MEXU). District Mixe, Totontepec Villa de Morelos, J. Rivera R. \& G. J. Martin 776 (MEXU), J. Rivera R. \& G. J. Martin 1090 (MEXU), E. Ramírez G. \& P. Ramírez C. 385 
(IEB), E. W. Nelson 831 (US). District Pochutla, Pluma Hidalgo, S. Acosta C. 1287 (UAMIZ); San Mateo Piñas, S. Acosta C. 1380 (UAMIZ); San Pedro Pochutla, C. Conzatti, B. P. Reko \& Makrinius 3246 (MEXU), B. P. Reko 6324 (GH), R. Torres C., J. L. Villaseñor, J. M. \& C. A. 5251 (MEXU). District Putla, Putla Villa de Guerrero, R. Torres C. \& A. García-Mendoza 6735 (MO). District Tehuantepec, Guevea de Humboldt, A. Campos V. 3873 (IEB); Santiago Lachiguiri, M. Cházaro B. \& M. Leach 3378 (XAL). District Teotitlán, Eloxochitlán de Flores Magón, R. E. Schultes \& B. P. Reko 404 (GH); Huautla de Jiménez, T. B. Croat 48312 (MO), R. E. Schultes \& B. P. Reko 320 (ECON). District Tuxtepec, San Juan Bautista Valle Nacional, A. R. López-Ferrari, A. Espejo, J. Ceja, A. Mendoza R., I. Reyes J. \& B. Pérez G. 2122 (UAMIZ).

10 Catopsis subulata L. B. Sm., Contr. Gray Herb. 114: 5, t. 1, f. 12.1936.

Fig. 9 Specimens examined: District Ixtlán, Ixtlán de Juárez, R. E. Schultes 663A (GH(x2)). District Juchitán, Asunción Ixtaltepec, R. Torres C. \& C. Martínez 6088 (MEXU); San Miguel Chimalapa, S. Maya J. 1760 (MEXU). District Tehuantepec, Magdalena Tequisistlán, C. Martínez R. 1401 (IEB, MEXU).

11 Catopsis wawranea Mez, in C. DC., Monogr. Phan. 9: 626. 1896.

Fig. 9

Specimens examined: District Tuxtepec, Santa María Jacatepec, A. R. López-Ferrari, A. Espejo \& G. Carnevali 3189 (UAMIZ), A. R. López-Ferrari, A. Espejo \& G. Carnevali 3190 (UAMIZ).

\section{FostereLLA}

1 Fosterella micrantha (Lindl.) L. B. Sm., Phytologia 7: 171, t. 1, f. 1-5. $1960 . \quad$ Fig. 10 Specimens examined: District Choapam, Santiago Choapam, $R$. Torres $C$. \& L. Cortés A. 9302 (MEXU, US); Santiago Yaveo, Y. Mexia 9187a (UC, US). District IxtLÁn, Santiago Comaltepec, G. Martínez C. 735 (ENCB, MEXU(x2), MO, XAL). District JuchiTÁN, Santa María Chimalapa, H. Hernández G. 798 (UAMIZ), H. Hernández G. 932 (UAMIZ). District Juquila, San Gabriel Mixtepec, R. Torres C. \& M. A. Martínez 6611 (MEXU, MO). District Pochutla, Candelaria Loxicha, A. R. López-Ferrari, A. Espejo \& A. Flores C. 570 (ENCB, IEB, MEXU, UAMIZ), E. J. Alexander 453 (MICH); San Miguel del Puerto, M. Cerón C., S. Salas M., E. Torres B. \& R. García S. 355 (UAMIZ), S. Salas M., J. Rivera H. \& M. Elorsa C. 2696 (MEXU), J. Rivera H., S. Salas M. \& M. Elorsa C. 2177 (MEXU); San Pedro Pochutla, W. R. Ernst 2519 (MEXU(X2), MICH, US), C. V. Morton \& E. Makrinius 2395 (US), J. Rivera H., S. Salas M. \& M. Elorsa C. 1105 (SERO). District Tehuantepec, Guevea de Humboldt, $R$. Torres C., L. Cortés A. \& M. P. Ramírez 9156 (IEB, MEXU), R. Torres C. \& A. Campos V. 13884 (FCME, MEXU, MO). District Tuxtepec, San Felipe Usila, J. I. Calzada, M. Vargas \& E. Ibarra 16824 (MEXU), R. E. Schultes \& B. P. Reko 660 (GH); San Juan Bautista Valle Nacional, T. B. Croat \& D. P. Hannon 65511 (MEXU, MO(x2). District Villa Alta, Tanetze de Zaragoza, C. Juergensen 389 (K, according Baker, 1889 as Cottendorfia neogranatensis). Without precise locality, C. Reiche $566 a(\mathrm{GH}, \mathrm{M})$. 
Espejo-Serna et al.: Bromeliad flora of Oaxaca

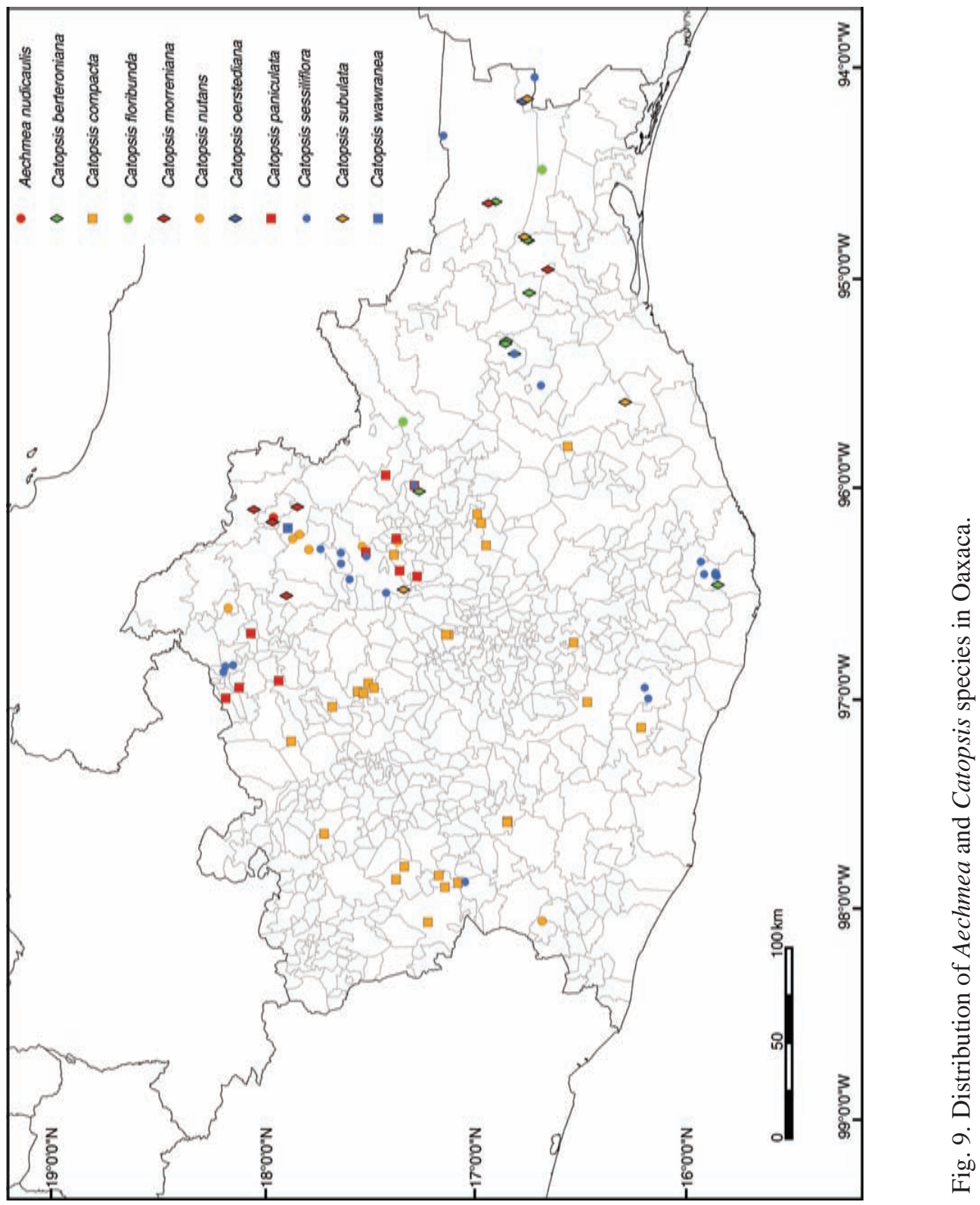


6 GREIGIA

1 Greigia juareziana L. B. Sm., Bull. Bromeliad Soc. 9: 51-52, f. $1959 . \quad$ Fig. 10 Specimens examined: District Ixtlán, Ixtlán de Juárez, Y. Arellanes C., J. García R. \& R. García S. 204 (MEXU); San Juan Atepec, M. B. Foster \& O. Van Hyning 3031 (MO); Santiago Comaltepec, G. Davidse, M. Sousa, O. Téllez, E. Martínez \& J. Davidse 30227 (MEXU), A. Espejo, A. R. López-Ferrari, J. Ceja, A. Mendoza R. \& G. Carnevali 6688 (UAMIZ(X5)), A. Espejo, A. R. López-Ferrari, J. Ceja, A. Mendoza R. \& G. Carnevali 6689 (UAMIZ), A. R. López-Ferarri, A. Espejo \& G. Carneval1 3201 (UAMIZ(X3)), O. van Hyning 5962 (US), O. van Hyning 5958 (US). District Mixe, Totontepec Villa de Morelos, D. H. Lorence, A. García-Mendoza \& R. Torres C. 4368 (ENCB, MEXU, XAL), J. Rivera R. \& G. J. Martin 1150 (MEXU), J. Rivera R. 3255 (UAMIZ), E. Velasco L. \& G. J. Martin 74 (MEXU), E. Velasco L. \& G. J. Martin 194 (MEXU). District Villa Alta, Santa María Yalina, J. Ceja, A. Espejo, A. R. López-Ferrari, A. Mendoza R. \& G. Carnevali 1605 (UAMIZ(X5)), A. R. López-Ferrari, A. Espejo, J. Ceja, A. Mendoza R. \& G. Carnevali 3096 (UAMIZ(X2)), A. R. López-Ferrari, A. Espejo, J. Ceja, A. Mendoza R. \& G. Carnevali 3097 (UAMIZ(X3)).

2 Greigia van-hyningii L. B. Sm., Bull. Bromeliad Soc. 9: 53, f. 1959.

Fig. 10 Specimens examined: District Tuxtepec, San Felipe Usila, A. Rincón G. \& C. Gallardo H. 484 (MEXU, XAL).

\section{GuZMANiA}

1 Guzmania nicaraguensis Mez \& C. F. Baker, in Mez, Bull. Torrey Bot. Club 30: 436-437. 1903.

Fig. 10

Specimens examined: District Choapam, Santiago Choapam, R. E. Schultes \& B. P. Reko 908 (GHx2)). District Juchitán, Matías Romero, T. B. Croat \& D. P. Hannon 63181 (MEXU); San Miguel Chimalapa, T. MacDougall 217 (US); Santa María Chimalapa, $H$. Hernández G. 884 (UAMIZ), H. Hernández G. 1163 (UAMIZ), T. MacDougall 732 ([S. \& D., 1979] US). District Mixe, San Juan Mazatlán, T. MacDougall s. n. ([S. \& D., 1979] US). District Tuxtepec, Acatlán de Pérez Figueroa, E. Guízar N. \& J. C. Echevarría 5717 (CHAP).

8 HechTiA

1 Hechtia caudata L. B. Sm., Phytologia 8: 5, t. 1, f. 1-5. 1961. Endemic. $\quad$ Fig. 10 Specimens examined: District Juchitán, San Pedro Tapanatepec, M. B. Foster \& O. Van Hyning 2999 ([S. \& D., 1979] US(x2)). District Tehuantepec, Santo Domingo Tehuantepec, M. L. Torres C., R. Torres C., P. Tenorio L. \& C. Martínez 326 (MEXU, MO), M. L. Torres C., R. Torres C., O. Téllez \& C. Martínez 388 (MEXU), M. L. Torres C., R. Torres C., C. Martínez \& J. Martínez 805 (MEXU, MO), M. L. Torres C., R. Torres C., L. Cortés A. \& C. Martínez 909 (MEXU, MO), R. Torres C. \& E. Martínez S. 11276 (MEXU). 
2 Hechtia confusa L. B. Sm., Contr. Gray Herb. 117: 22, t. 1, f. 71, 72. $1937 . \quad$ Fig. 10 Specimens examined: District Coixtlahuaca, Tepelmeme Villa de Morelos, $R$. Cruz C. 2179 (ENCB). District Huajuapam, Santiago Chazumba, A. Salinas T. 7659 (MEXU(x2)).

3 Hechtia conzattiana L. B. Sm., Contr. Gray Herb. 117: 19, t. 1, f. 56. $1937 . \quad$ Fig. 10 Specimens examined: District Coixtlahuaca, Tepelmeme Villa de Morelos, A. Salinas T. 7712 (MEXU). District Cuicatlán, Cuyamecalco Villa de Zaragoza, C. Conzatti \& I. Gómez 3501 (MEXU, US). District Teotitlán, Santa María Tecomavaca, A. Salinas T., Mark Newman \& A. Ocampo 7065 (MEXU(x2)); Teotitlán de Flores Magón, A. Salinas T., G. Flores F. \& E. Martínez C. 4235 (MEXU).

4 Hechtia fosteriana L. B. Sm., Phytologia 8: 8, t. 1, f. 10-11. 1961. Endemic. Fig. 10 Specimens examined: District Tehuantepec, Magdalena Tequisistlán, $M$. B. Foster \& $O$. Van Hyning 2935 ([S. \& D., 1979] US(x2)); Santa María Guienagati, A. Campos V. \& R. Torres C. 3554 (MEXU), A. Campos V. \& R. Torres C. 3555 (MEXU, MO).

5 Hechtia fragilis Burt-Utley \& Utley, Brittonia 39: 40-41, f. 2. 1987.

Fig. 10 Specimens examined: District Cuicatlán, San Juan Bautista Cuicatlán, A. García-Mendoza, R. Medina L. \& J. Sandoval 7379 (MEXU), F. González Medrano, V. Jaramillo, J. L. Villaseñor, P. Ruíz \& S. Singer F-1109 (MEXU, TEX), R. Medina L., L. Alvarado C. \& J. Sandoval 1036 (MEXU(x2)), A. Salinas T. \& M. Way 8057 MEXU(x2)), A. Salinas T. \& E. Martínez C. 8112 (MEXU). District Teotitlán, Santa María Ixcatlán, A. Salinas T. \& E. Martínez C. 8082 (MEXU(x2); Teotitlán de Flores Magón, J. Utley \& K. Burt-Utley 6980 (NY according Burt-Utley \& Utley, 1987, MEXU).

6 Hechtia galeottii Mez, Repert. Spec. Nov. Regni Veg. 16: 71-72. 1919. EndEMic. Fig. 10 Specimens eXamined: District Etla, San Francisco Telixtlahuaca, C. G. Pringle 6703 (BR, ENCB, GH, MEXU, P(x2), UC, VT); San Jerónimo Sosola, A. Salinas T. \& E. Martínez C. 6256 (MEXU(x2)); San Juan Bautista Atatlahuaca, A. Espejo, A. R. López-Ferrari, J. Ceja, A. Mendoza R. \& I. Ramírez M. 6510 (CICY(x3), UAMIZ(x5)), A. Espejo, A. R. LópezFerrari, J. Ceja, A. Mendoza R. \& I. Ramírez M. 6511 (UAMIZ(x3)). District NochixtLán, San Mateo Etlatongo, A. García-Mendoza \& J. Reyes 5151 (MEXU). Without precise locality, H. Galeotti 5440 ([S. \& D., 1979] B).

7 Hechtia lanata L. B. Sm., Phytologia 8: 5-6, t. 1, f. 6-9. 1961. Endemic.

Fig. 11 Specimens examined: District Tehuantepec, Magdalena Tequisistlán, M. B. Foster \& O. Van Hyning 2934 ([S. \& D., 1979] US).

8 Hechtia lyman-smithii Burt-Utley \& Utley, Brittonia 39: 37-40, f. 1. 1987. ENDEMIC.

Fig. 11 Specimens examined: District Cuicatlán, San Juan Bautista Cuicatlán, A. Espejo, A. $R$. López-Ferrari, J. Ceja, A. Mendoza R. \& I. Ramírez M. 6514 (UAMIZ), I. Ramírez M., A. Espejo, A. R. López-Ferrari, A. Mendoza R. \& J. Ceja 1038 (CICY). District JuxtlahuaCa, 


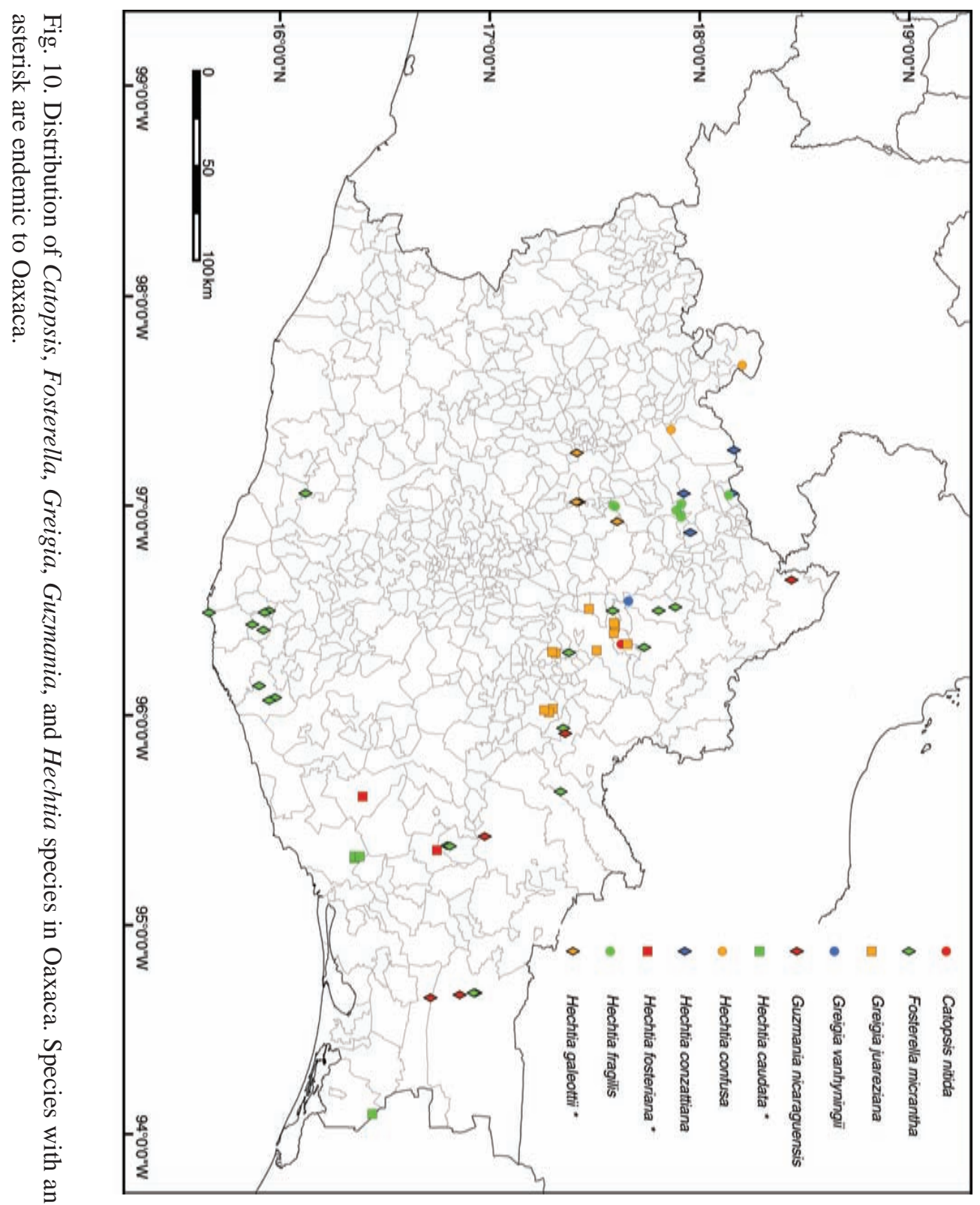


Santos Reyes Tepejillo, J. I. Calzada 19879 (MEXU). District Teotitlán, San Antonio Nanahuatipam, A. García-Mendoza, A. Salinas T. \& E. Martínez S. 3467 (MEXU), A. Salinas T., E. Martínez S. \& A. García-Mendoza 4190 (MEXU, MO), A. Salinas T., A. Ocampo \& A. Ramírez R. 7414 (MEXU); Santa María Ixcatlán, J. Utley \& K. Burt-Utley 6982 (MEXU).

9 Hechtia marnier-lapostollei L. B. Sm., Bull. Bromeliad Soc. 11: 58, f. 1961. EndEMic.

Fig. 11

Specimens eXamined: District Juchitán, El Barrio de la Soledad, S. Zamudio 6356 (IEB). District Yautepec, San Carlos Yautepec, E. Matuda 38429 (MEXU); San Juan Lajarcia, R. Torres C., L. Cortés A. \& C. Martínez 9859 (MEXU). Without precise locality, Schwartz sub J. Marnier-Lapostolle (US).

10 Hechtia nuusaviorum Espejo \& López-Ferrari, Acta Bot. Mex. 78: 98-103, f. 1-3. 2007. ENDEMIC.

Fig. 12 Specimens examined: District Tlaxiaco, Santiago Yosondúa, J. Ceja, A. Espejo, A. R. López-Ferrari, A. Mendoza R. \& G. Carnevali 1751 (UAMIZ(x6)); A. García-Mendoza, A. de Ávila, L. Cervantes \& A. Saynes 6841 (MEXU), B. \& K. Benz, B. Hallberg \& M. Burd 679 (MEXU).

11 Hechtia podantha Mez, in C. DC., Monogr. Phan. 9: 549-550. 1896.

Fig. 11 Specimens examined: District Huajuapam, Huajuapam de León, M. C. Carlson 2175 (US), A. García-Mendoza, A. de Ávila, L. Cervantes \& A. Saynes 6846 (MEXU). District SilAcAYOAPAM, Silacayoapam, E. Guízar N. \& A. Castañeda 3779 (CHAP, MEXU, UAMIZ). District Teotitlán, San Antonio Nanahuatipam, A. García-Mendoza, A. Salinas T. \& E. Martínez S. 3466 (MEXU); Santa María Ixcatlán, A. Salinas T., E. Martínez C. \& R. Martínez S. 6426 (MEXU). District Teposcolula, Santa María Chilapa de Díaz, D. $H$. Lorence \& A. García-Mendoza 4808 (ENCB, MEXU), R. Torres C. \& A. García-Mendoza 6679 (MEXU).

12 Hechtia pringlei B.L. Rob. \& Greenm., Amer. J. Sci. ser. 3. 50: 167. 1895. EndEMic.

Fig. 11

Specimens examined: District Centro, San Antonio de la Cal, C. G. Pringle 4637 (BM, BR, ENCB, GOET, MEXU, NY, P, UC, US(x2), VT, Z). District Cuicatlán, San Juan Bautista Cuicatlán, C. Conzatti \& V. González 1167 (MEXU). District EtLA, San Agustín Etla, V. Mateos R. 26 (MEXU). District Ixtlán, Capulalpam de Méndez, S. Figueroa B. \& F. Y. Guzmán R. 201 (UAMIZ(x3)), S. Figueroa B. \& F. Y. Guzmán R. 533 (UAMIZ(x6); San Pablo Macuiltianguis, R. Ortega O. 983 (XAL). District Miahuatlán, San Juan Mixtepec, E. Hunn OAX-1435 (MEXU). District Tlacolula, San Jerónimo Tlacochahuaya, A. García-Mendoza, L. Torres \& L. Cortés A. 2867 (MEXU), J. Rzedowski 33019 (ENCB(x2)); Tlacolula de Matamoros, J. Schoenwetter JSOX-100 (ENCB).

13 Hechtia rosea E. Morren ex Baker, Handb. Bromel. 140. $1889 . \quad$ Fig. 11 Specimens examined: District Juchitán, Asunción Ixtaltepec, E. Pérez G. \& B. Pérez Reyes 721 (MEXU), E. Pérez G. \& B. Pérez Reyes 1227 (MEXU), E. Pérez G. \& B. Pérez Reyes 
1293 (MEXU), S. Salas M. 4680 (MEXU, UAMIZ), A. Saynes V., M. Elorsa C., S. Salas M. Kenia \& Gonzalo 3067 (MEXU, UAMIZ), A. Saynes V., A. Sánchez \& S. Salas H. 3249 (MEXU), A. Saynes V., A. Sánchez \& S. Salas H. 3331 (MEXU), A. Saynes V. \& A. Sánchez 3581 (SERO), A. Saynes V. \& A. Sánchez 3591 (MEXU, SERO); Ciudad Ixtepec, T. MacDougall 2 (MEXU), C. Martínez R. 1112 (MEXU). J. Meave del C. \& E. Pérez G. 1906 (MEXU(x2)); Juchitán de Zaragoza, D. H. Lorence \& R. Cedillo T. 3005B (MEXU); San Juan Guichicovi, I. Ramírez M. \& G. Carnevali 924 (CICY). District Tehuantepec, Magdalena Tequisistlán, G. Flores F. \& I. Calzada 3614 (MEXU); Salina Cruz, C. Martínez R. 245 (MEXU); San Miguel Tenango, R. Torres C. \& C. Martínez 10516 (MEXU); San Pedro Huamelula, S. Salas M. 831 (UAMIZ); Santa María Jalapa del Marqués, $R$. Torres C. \& P. Tenorio L. 12922 (MEXU); Santiago Laollaga, R. Torres C. \& C. Martínez 5840 (MEXU, MO), R. Torres C. \& A. Campos V. 14030 (MEXU, MO), R. Torres C. \& A. Campos V. 14031 (MEXU); Santo Domingo Tehuantepec, O. Dorado, A. Liston \& D. Arias 1640 (MEXU), A. García-Mendoza, D. H. Lorence \& R. Torres C. 1357 (MEXU, MO), C. Martínez R. 286 (MEXU), C. Martínez R. 1140 (MEXU), C. Martínez R. 1680 (MEXU), C. Martínez R. 1768 (MEXU), M. L. Torres C., R. Torres C. \& C. Martínez 650 (MEXU, MO), M. L. Torres C., R. Torres C. \& C. Martínez 685 (MEXU, MO), R. Torres C., D. H. Lorence \& A. García-Mendoza 4062 (FCME, MEXU, MO), R. Torres C. 4238 (FCME, MEXU) R. Torres C., L. Cortés A. \& C. Martínez 9769 (MEXU). District Yautepec, San Bartolo Yautepec, F. Liebmann 31 ([S. \& D., 1979] C); Santa María Ecatepec, T. MacDougall s. n. ([S. \& D., 1979] US).

14 Hechtia roseana L. B. Sm., Contr. Gray Herb. 117: 17, t. 1, f. 41, 42. $1937 . \quad$ Fig. 11 Specimens examined: District Etla, San Jerónimo Sosola, A. Espejo, A. R. López-Ferrari \& J. Ceja 5319 (UAMIZ(x2)). District Teotitlán, San Juan de los Cues, A. R. López-Ferrari, A. Espejo, J. García-Cruz \& E. Yáñez G. 1826 (MEXU(x3), UAMIZ(x6)), A. R. LópezFerrari, A. Espejo, J. García-Cruz \& E. Yáñez G. 1827 (UAMIZ(x2)).

15 Hechtia sphaeroblasta B.L. Rob., Proc. Amer. Acad. Arts 35: 323. $1900 . \quad$ Fig. 11 Specimens examined: District Huajuapam, San Pedro y San Pablo Tequixtepec, A. Aguilar S. 31 (CHAP); Santiago Chazumba, A. G. Miranda M. 881 (CHAP, MEXU, UAMIZ); Santo Domingo Tonalá, E. Guízar N. \& A. Castañeda 3658 (CHAP, MEXU). District Juxtlahuaca, San Juan Mixtepec, J. Reyes S. 2152 (MEXU). District Silacayoapam, Silacayoapam, E. Guízar N. \& A. Castañeda 3781 (CHAP; MEXU, UAMIZ(x2). District Teposcolula, Villa de Tamazulapam del Progreso, J. Rzedowski 1969 (ENCB, MEXU), K. Torke, D. \& LeDoux 425 (ENCB).

16 Hechtia sp. 1. ENDEMIC.

Specimens examined: District Juxtlahuaca, San Juan Mixtepec, J. Reyes S. 1149 (MEXU).

17 Hechtia sp. 2. ENDEMIC.

Specimens examined: District Tlaxiaco, Santiago Yosondúa, J. Ceja, A. Espejo, A. R. López-Ferrari, A. Mendoza R. \& G. Carnevali 1759 (UAMIZ(x6)), A. García-Mendoza, A. de Ávila, L. Cervantes \& A. Saynes $6842(\mathrm{MEXU}(\mathrm{x} 2))$. 
18 Hechtia sp. 3. ENDEMIC.

Fig. 11

Specimens examined: District Teotitlán, San Antonio Nanahuatipam, P. Tenorio L., L. Alvarado \& E. Martínez 20449 (MEXU), P. Tenorio L., L. Alvarado \& E. Martínez 20453 (MEXU).

19 Hechtia sp. 4. ENDEMIC.

Fig. 11

Specimens examined: District Juxtlahuaca, Santos Reyes Tepejillo, J. I. Calzada 22581 (MEXU(X2)). District Teposcolula, San Andrés Lagunas, A. Espejo, A. R. López-Ferrari, J. Ceja, A. Mendoza R. \& G. Carnevali 6852 (UAMIZ(x6)); San Pedro y San Pablo Teposcolula, A. García-Mendoza 183 (MEXU), R. Cedillo T., D. H. Lorence \& A. GarcíaMendoza 773 (MEXU); Villa Tejupam de la Unión, J. I. Calzada 23830 (MEXU).

20 Hechtia sp. 5. ENDEMIC.

Fig. 11

Specimens examined: District Yautepec, San Carlos Yautepec A. Salinas T. \& E. Martínez C. 8176 (IEB, MEXU); Santa María Ecatepec, P. Tenorio L. 18866 (MEXU).

9 HOHENBERGIOPSIS

1 Hohenbergiopsis guatemalensis (L. B. Sm.) L. B. Sm. \& Read, Phytologia 33: 440. 1976.

Fig. 12

Specimens examined: District Ixtlán, Ixtlán de Juárez, J. Rzedowski 33830 (ENCB(x2)), J. Santana C. \& L. Pacheco 907 (UAMIZ(x3)); Santiago Comaltepec, C. R. Beutelspacher B. 68 (MEXU), C. R. Beutelspacher B. 79 (ENCB, MEXU(x2)), G. Davidse, M. Sousa, O. Téllez, E. Martínez \& J. Davidse 30224 (MEXU(x2)), M. B. Foster \& O. Van Hyning 3003 (US(x2)), E. Martínez S., O. Téllez, G. Davidse \& J. Davidse 8731 (MEXU(x2)), G. Pérez Pablo \& E. Martínez S. 50 (MEXU), R. Torres C. \& L. Cortés A. 11670 (MEXU). Without municipio indicated, Sierra de Juárez, E. Matuda 38703 (MEXU).

10 PITCAIRNIA

1 Pitcairnia heterophylla (Lindl.) Beer, Fam. Bromel. 68. 1857.

Fig. 12

Specimens examined: District Juchitán, San Miguel Chimalapa, S. Maya J. 4352 (MEXU), R. Torres C. \& C. Martínez 4863 (MEXU); Santa María Chimalapa, J. Rivera H., R. García S. \& J. C. Flores V. 1194 (MEXU); Santo Domingo Petapa, A. Campos V., R. Torres C. \& L. Cortés A. 1761 (MEXU). District Juquila, San Juan Lachao, W. Boege 3028 A (MEXU); Santa Catarina Juquila, C. Conzatti 4357 (GH, US), C. Conzatti 4528 (GH, MEXU, US). District Miahuatlán, San Jerónimo Coatlán, A. Campos V. 2732 (MEXU), A. Campos V. 3482 (MEXU), R. Torres C., L. Cortés A. \& A. Campos V. 11901 (MEXU). District Mixe, Totontepec Villa de Morelos, J. Rivera R. JR3367 (MEXU). District PochutLA, San Miguel del Puerto, J. Pascual 952 (UAMIZ); San Pedro el Alto, T. B. Croat 46117 (MO). District Putla, Putla Villa de Guerrero, A. Espejo, A. R. López-Ferrari, J. Ceja \& A. Mendoza R. 6793 (UAMIZ). District Tehuantepec, Guevea de Humboldt, A. Campos V. \& R. Torres C. 3592 (FCME, MEXU), R. Torres C., L. Cortés A. \& M. P. Ramírez 9139 (MEXU); Santa María Guienagati, P. Tenorio L., R. Torres C. \& C. Martínez 11165 (IEB, 


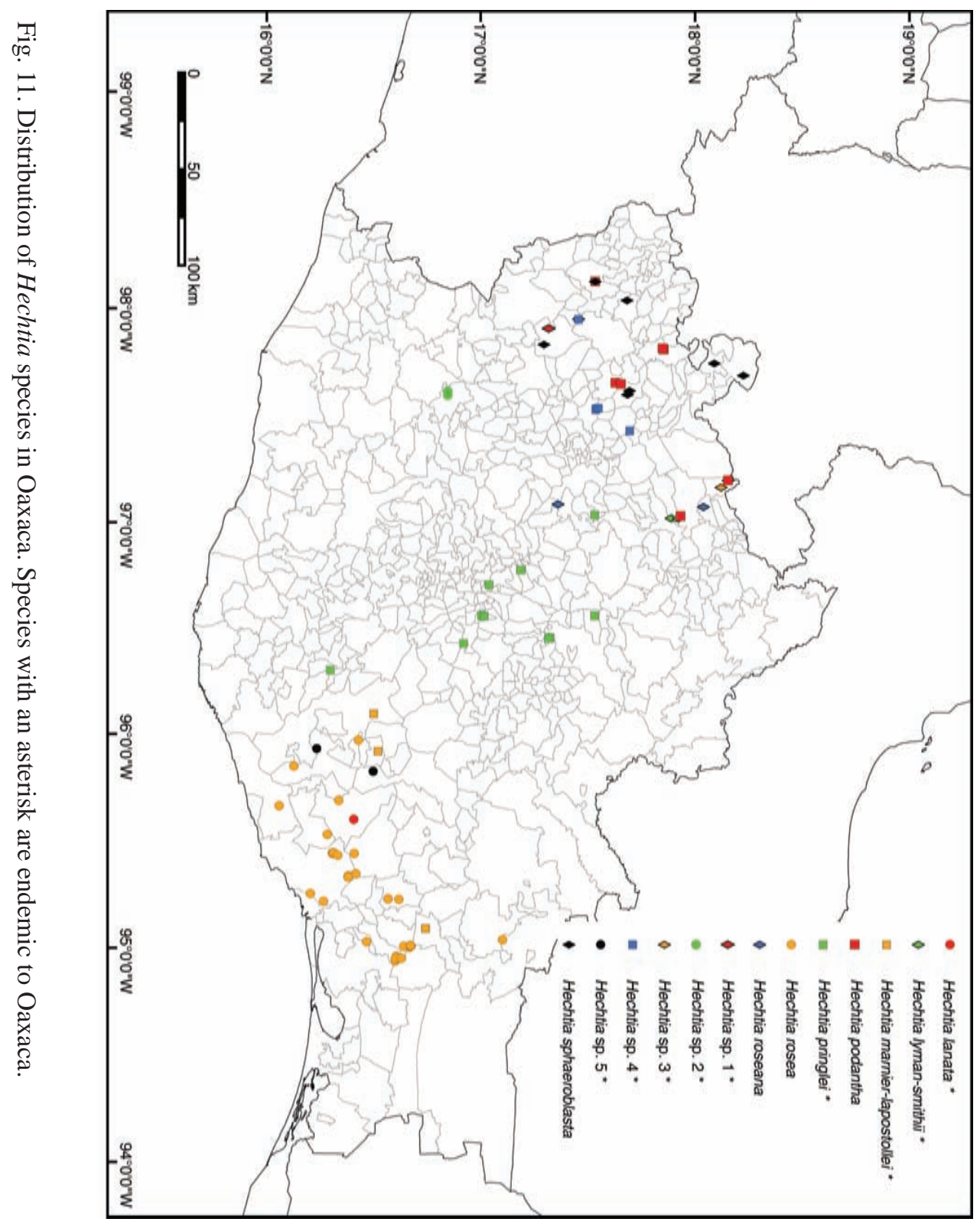


MEXU). District Teotitlán, Huautla de Jiménez, R. Torres C. \& M. A. Martínez 6556 (IEB, MEXU, MO).

2 Pitcairnia imbricata (Brongn.) Regel, Gartenflora 17: 135, t. 579. 1868.

Fig. 12

Specimens examined: District Choapam, San Juan Comaltepec, R. Torres C. \& L. Cortés A. 10441 (MEXU); San Juan Lalana, F. Liebmann 71 ([S. \& D., 1979] C); Santiago Jocotepec, B. P. Reko 4128 (US). District IxtLÁn, Santiago Comaltepec, W. L. Graham 1414 (MICH(x2)), A. Mendoza R., B. Pérez G. \& R. Riba 280 (UAMIZ), J. Santana C. \& L. Pacheco 913 (UAMIZ(x3)), J. Utley \& K. Burt-Utley 6739 (MEXU(X2)). District Juchitán, Asunción Ixtaltepec, $R$. Torres C. \& C. Martínez 6087 (MEXU, MO). District Pochutla, San Pedro el Alto, A. García-Mendoza \& F. Martínez 2687 (MEXU, MO). District TehuANTEPec, Guevea de Humboldt, sin colector s. n. (MEXU), R. Torres C. \& C. Martínez 5948 (MO). District Tuxtepec, San Felipe Usila, G. Ibarra M., J. Meave \& M. Vargas 3748 (MEXU, MO); San Juan Bautista Valle Nacional, A. R. López-Ferrari, A. Espejo, J. Ceja, A. Mendoza R., B. Pérez G. \& I. Reyes J. 2117 (UAMIZ), A. R. López-Ferrari, A. Espejo \& G. Carnevali 3196 (UAMIZ), R. Torres C. \& L. Cortés A. 7250 (MEXU). District Villa Alta, San Juan Juquila Vijanos, X. Munn, R. Aragón, R. Guzmán, R. Manzano \& C. Gabriel 8 (MEXU(x2)).

3 Pitcairnia oaxacana L. B. Sm., Contr. Gray Herb. 117: 25-26, t. 2, f. 16, 17. 1937.

Fig. 12

Specimens examined: District Juquila, San Gabriel Mixtepec, A. R. López-Ferrari, A. Espejo, J. Ceja \& A. Mendoza R. 2942 (IEB(x2), UAMIZ(x2)). District Pochutla, San Miguel del Puerto, A. Campos V. 4787 (MEXU), E. Martínez S., M. Elorsa C., J. Castrejón \& D. Sánchez 33124 (UAMIZ), J. Rivera H., S. Salas M. \& E. Martínez S. 1829 (MEXU, SERO), S. Salas M. 3068 (MEXU); San Pedro Pochutla, B. P. Reko 3383 (US).

4 Pitcairnia recurvata (Scheidw.) K. Koch, Index Sem. Hort. Bot. Berol. 1857, App.: 4. 1857.

Fig. 12

Specimens examined: District Juchitán, Santa María Chimalapa, H. Hernández G. 259 (UAMIZ), R. Torres C. \& C. Martínez 6033 (MEXU, MO). District Teotitlán, Santa María Chilchotla, X. Munn, E. Juárez \& L. Juárez 1274 (MEXU). District Tuxtepec, Nuevo Soyaltepec, L. Cortés A. \& R. Torres C. 4 (ENCB, MEXU, MO, XAL), L. Cortés A. \& R. Torres C. 423 (MEXU), L. Cortés A., R. Torres C., M. Sousa S. \& G. Andrade M. 866 (MEXU), L. Cortés A. \& R. Torres C. 1067 (MEXU), A. García-Mendoza, L. Cortés A. \& L. Torres 2758 (MEXU); San Pedro Ixcatlán, J. I. Calzada 10334 (XAL).

5 Pitcairnia ringens Klotzsch, in Link, Klotzsch \& Otto, Icon. pl. rar. 63, t. 25. 1842.

Fig. 12

Specimens examined: District Ixtlán, Santiago Comaltepec, G. J. Martin 410 (MO, UC, US), O. van Hyning 5960 (US). District Mixe, Totontepec Villa de Morelos, J. Rivera R. \& G. J. Martin 1480 (MEXU). Without precise locality, H. Galeotti 4916bis ([S. \& D., 1979] BR). 
6 Pitcairnia schiedeana Baker, Handb. Bromel. 95. 1889.

Specimens examined: District Ixtlán, Santa María Yavesía, H. Galeotti 4913 (P).

7 Pitcairnia tuerckheimii Donn. Sm., Bot. Gaz. (Crawfordsville) 13: 190, t. 24. 1888.

Specimens examined: District Pochutla, Santa María Colotepec, S. Salas M. 3936 (UAMIZ).

8 Pitcairnia undulata (hort. ex Beer) Scheidw., in Otto \& A. Dietr., Allg. Gartenzeitung 10: 275. 1842.

Fig. 12

Specimens examined: District Juchitán, Santa María Chimalapa, $R$. de Santiago G., J. Rivera H., T. Alvarez R. \& D. Acuca V. 683 (UAMIZ), H. Hernández G. 1396 (UAMIZ), H. Hernández G. 1473 (MEXU, UAMIZ), J. Rivera H., E. Martínez S. \& C. Perret 1388 (MEXU).

9 Pitcairnia undulatosepala Rauh, Trop. Subtrop. Pflanzenw. 58: 16-19, f. 8-9. 1986, “undulato-sepala”.

Specimens examined: Without District and municipio indicated, Oaxaca-Tehuantepec, $W$. Rauh 52598 (HEID(x3).

10 Pitcairnia virginalis Utley \& Burt-Utley, Ann. Missouri Bot. Gard. 78: 267-269, f. 2. 1991. ENDEMIC.

Fig. 12

Specimens examined: District Jamiltepec, Santiago Pinotepa Nacional, $S$. D. Koch, $P$. A. Fryxell \& T. Wendt 79444 (ENCB, MEXU, MO, TEX, US(x2)). District Pochutla, Pluma Hidalgo, J. Utley \& K. Burt-Utley 8400 (paratipo CAS de acuerdo con Utley \& Utley, 1991); San Pedro el Alto, R. Torres C. \& A. García-Mendoza 6835 (MEXU), J. Utley \& K. Burt-Utley 8588 (MEXU, MICH, MO, US according Utley \& Utley, 1991). District Putla, Putla Villa de Guerrero, A. Espejo, A. R. López-Ferrari, J. Ceja \& A. Mendoza R. 6796 (UAMIZ(x5)).

\section{RACINAEA}

1 Racinaea adscendens (L. B. Sm.) M. A. Spencer \& L. B. Sm., Phtyologia 74: 153. 1993.

District IXTLÁN, Santiago Comaltepec, O. van Hyning 6054 ([S. \& D., 1979] US). DisTRICT Juchitán, San Miguel Chimalapa, M. Ishiki I. 1607 (MEXU). District MiXe, Totontepec Villa de Morelos, E. Ramírez G. \& P. Ramírez C. 395 (MEXU).

2 Racinaea ghiesbreghtii (Baker) M. A. Spencer \& L. B. Sm., Phtyologia 74: 153. 1993.

Specimens examined: District Ixtlán, Santiago Comaltepec, G. Davidse, M. Sousa, O. Téllez, E. Martínez \& J. Davidse 30226 (MEXU). District Juquila, San Juan Lachao, J. Rzedowski 19611 ([S. \& D., 1979], US). District Miahuatrán, San Jerónimo Coatlán, A. 
Espejo-Serna et al.: Bromeliad flora of Oaxaca

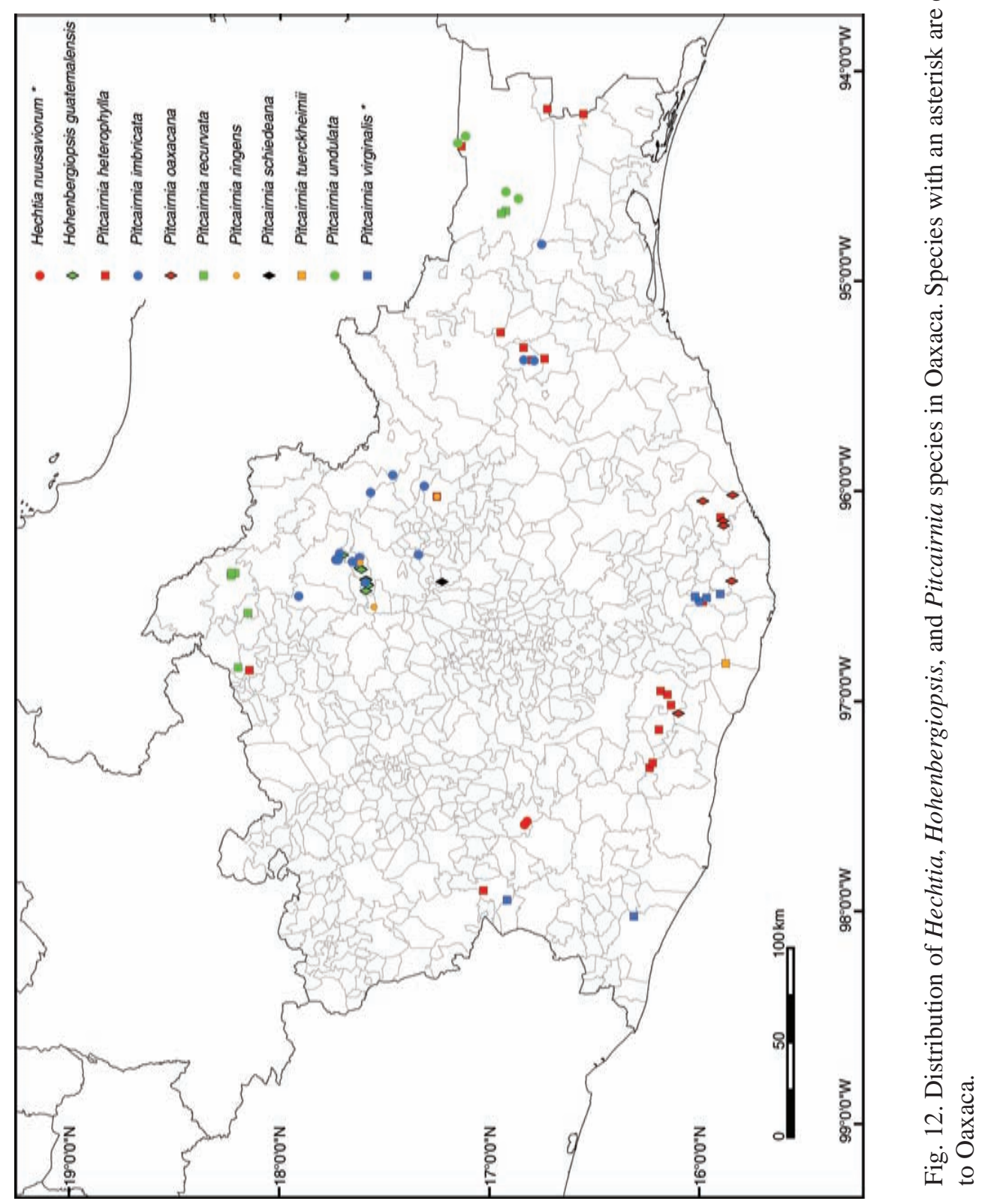


Campos V. \& R. Torres C. 1530 bis (MEXU). District Mixe, Totontepec Villa de Morelos, J. Rivera R. JR1228 (MEXU(x2)).

\section{TiLlandsia}

1 Tillandsia achyrostachys E. Morren ex Baker, Handb. Bromel. 171. $1889 . \quad$ Fig. 13 Specimens examined: District Centro, Tlalixtac de Cabrera, Torres C. \& A. García-Mendoza 8067 (MEXU). District Coixtlahuaca, Tepelmeme Villa de Morelos, A. Salinas T. 7722 (MEXU). District Cuicatlán, Concepción Pápalo, A. Salinas T. \& R. Martínez S. 5934 (MEXU); San Juan Bautista Cuicatlán, E. Martínez S., J. L. Rebolledo C. \& V. Torres H. 33414 (MEXU); San Pedro Jocotipac A. Salinas T. \& A. Reyes G. 4857 (MEXU); Santos Reyes Pápalo, R. Torres C. \& J. L. Villaseñor 5059 (MEXU, MO). District EtLA, San Juan Bautista Guelache, T. MacDougall s. n. (US(x2)); San Juan del Estado, G. J. Martin GJM-M267 (MEXU, MO); San Pablo Etla, C. Conzatti \& V. González 947 (GH, MEXU). District Huajuapam, Huajuapam de León, A. García-Mendoza, A. Campos V. \& J. Reyes 3620 (IEB, MEXU); San Andrés Dinicuiti W. Boege 3018 (MEXU); Santo Domingo Tonalá, A. Espejo, A. R. López-Ferrari, J. Ceja, A. Mendoza R. \& G. Carnevali 6875 (UAMIZ). District Ixtlán, Capulalpam de Méndez, S. Figueroa B. \& F. Y. Guzmán R. 124 (UAMIZ), S. Figueroa B. \& F. Y. Guzmán R. 372 (CHAP); San Juan Chicomezúchil, A. R. López-Ferrari, A. Espejo, J. Ceja, A. Mendoza R. \& G. Carnevali 3080 (UAMIZ); San Juan Quiotepec, J. García G. 76 (MEXU, UAMIZ); Santa Catarina Lachatao, H. H. Iltis 27142 (MEXU), T. MacDougall s. n. (US). District Juxtlahuaca, San Juan Mixtepec, J. Reyes S. 1358 (MEXU); Santiago Juxtlahuaca M. Cházaro B., M. Kimnach \& M. Negrete A. 7110 (MEXU). District Sola de Vega, Santa María Sola, V. García G. 11 (UAMIZ). District Teotitlán, Santa María Ixcatlán A. Salinas T., E. Martínez C. \& R. Martínez S. 6464 (MEXU); Teotitlán de Flores Magón, J. Bauml \& M. Kimnach 378 (US). District Teposcolula, Villa de Tamazulapam del Progreso, A. García-Mendoza, F. Martínez \& F. Mérida 2043 (CHAP, MEXU, MO), J. Rzedowski 19691 (ENCB), R. Torres C., A. GarcíaMendoza \& L. Cortés A. 9588 (MEXU). District Tlacolula, San Jerónimo Tlacochahuaya, A. García-Mendoza, L. Cortés A. \& L. Torres 2831 (MEXU); San Lorenzo Albarradas, J. Ceja, A. Espejo, A. R. López-Ferrari \& A. Mendoza R. 1578 (UAMIZ); San Pablo Villa de Mitla, A. Espejo, A. R. López-Ferrari, I. Ramírez M. \& G. Carnevali 6754 (UAMIZ), R. Torres C. \& A. Campos V. 10701 (MEXU); San Pedro Totolapa, E. W. Nelson 2545 (US); Tlacolula de Matamoros, A. Espejo \& A. R. López-Ferrari 6761 (UAMIZ). District ViLla Alta, Villa Hidalgo, R. E. Gereau \& A. J. Moyer 2171 (ENCB, MEXU, MO). District YautePec, San Carlos Yautepec, T. MacDougall sub E. Matuda 26071 (US), E. Matuda \& colaboradores 38536 (LL, MEXU, US). Without precise locality, T. MacDougall s. $n$. (MICH), E. Torres B. \& S. Salas M. 1813 (MEXU).

2 Tillandsia atenangoensis Ehlers \& Wülfinghoff, Bromelie 2001 (2): 45, figs. 2001. EndEMIC.

Specimens examined: District Huajuapam, Santo Domingo Tonalá, A. Espejo, A. R. LópezFerrari, J. Ceja, A. Mendoza R. \& G. Carnevali 6874 (UAMIZ). District Silacayoapam, San Agustín Atenango, R. Ehlers \& K. Ehlers EM930603 (MEXU, WU(2)), R. Ehlers et 
al. EM980101 (WU), W. Wulfinghoff Wü88-312 (WU), K. Ehlers \& R. Ehlers 8075 (WU), J. Lautner L524 (WU).

3 Tillandsia atroviolacea Ehlers \& Koide, J. Bromeliad Soc. 47: 21. 1997. EndEMic.

Fig. 13

Specimens examined: District Tlaxiaco, San Mateo Peñasco, J. Ceja, A. Espejo, A. R. López-Ferrari, A. Mendoza R. \& G. Carnevali 1766 (UAMIZ), K. Ehlers \& R. Ehlers EM 930901 (WU(x6)), P. Koide \& A. Lau 91-90285 (MEXU).

4 Tillandsia balbisiana Schult. \& Schult.f., Syst. veg. 7: 1212. 1830.

Fig. 13

Specimens examined: District Juchitán, Santo Domingo Ingenio, M. B. Foster \& O. Van Hyning 2979 (US).

5 Tillandsia belloensis W. Weber, Feddes Repert. 94: 602. 1983.

SPECIMENS EXAMINED: Without precise locality, E. Matuda 38540 (LL).

6 Tillandsia bourgaei Baker, J. Bot. 25: 278. 1887.

Fig. 13

Specimens examined: District Coixtlahuaca, San Pedro Nopala, R. Cruz C. $1907 a$ (ENCB(x2)), A. García-Mendoza, R. Torres C. \& L. Cortés A. 3074 (MEXU), R. Torres C., A. García-Mendoza \& L. Cortés A. 9557 (MEXU(x2)); Tepelmeme Villa de Morelos, A. Salinas T., D. Frame, P. Tenorio L., A. García-Mendoza, E. López \& E. López F-3371 (MEXU); Tlacotepec Plumas, R. Cruz C. 2312 (ENCB). District Etla, San Francisco Telixtlahuaca, A. Espejo, A. R. López-Ferrari, J. Ceja, A. Mendoza R. \& I. Ramírez M. 6502 (UAMIZ(x2)). District Ixtlán, Capulalpam de Méndez, D. H. Lorence, A. García-Mendoza \& R. Cedillo T. 3592 (MEXU, MO), G. J. Martin 511 (ENCB, MEXU, MO, UC); Ixtlán de Juárez, A. R. López-Ferrari, A. Espejo, J. Ceja, A. Mendoza R. \& G. Carnevali 3086 (UAMIZ(x2)), R. Merrill King 2093 (MICH); Santa Catarina Ixtepeji, H. H. Iltis 27135 (MEXU(x2)), T. Macdougall s. n. (GH, US); Santa Catarina Lachatao, T. MacDougall 3-a (US). District Juxtlahuaca, San Juan Mixtepec, J. Reyes S. 220 (MEXU), J. Reyes S. 1448 (MEXU). District Nochixtlán, Asunción Nochixtlán, O. van Hyning 6047 (US); Santo Domingo Yanhuitlán, A. Espejo, A. R. López-Ferrari, J. Ceja, A. Mendoza R. \& G. Carnevali 6844 (UAMIZ), H. H. Iltis, R. Koeppen \& F. Iltis 1146 (ENCB, MICH, US(x2)). District Teposcolula, San Juan Teposcolula, A. García-Mendoza 867 (MEXU(x2)); San Pedro Nopala, A. Salinas T. \& P. Tenorio L. 5835 (MEXU); San Sebastián Nicananduta, J. I. Calzada 23835 (MEXU), J. I. Calzada 23899 (MEXU); Villa Tejupam de la Unión, A. García-Mendoza, F. Martínez \& F. Mérida 2091 (MEXU, UAMIZ), A. García-Mendoza, R. Torres C. \& L. Cortés A. 3085 (MEXU), R. Torres C., A. García-Mendoza \& L. Cortés A. 9567 (MEXU(x2)), R. Torres C., A. García-Mendoza \& L. Cortés A. 9569 (MEXU). District Tlacolula, San Pablo Villa de Mitla, M. Cházaro B., M. Kimnach \& M. Negrete A. 6789 (MEXU, TEX, XAL); Santa Ana del Valle, A. García-Mendoza, L. Torres \& L. Cortés A. 2892 (MEXU(x2); Santo Domingo Albarradas, A. Espejo, A. R. López-Ferrari, J. Ceja \& A. Mendoza R. 6680 (UAMIZ(x3)); Villa Díaz Ordaz, T. MacDougall 356 (US(x2)). District Tlaxiaco, Heroica Ciudad de Tlaxiaco, E. Matuda 38292 (MEXU(x2), MO(x2), US). District Villa Alta, Santo Domingo Xagacía, R. E. Gereau \& A. J. Moyer 2182 
(MEXU(x2), MO(x3)). Without precise locality, A. Lau Jr. sub D. Cathcart s. n. (SEL), G. J. Martin GJM-M293 (MEXU), E. Matuda 28469 (MEXU), E. Matuda 38548 (LL, MEXU, US), E. Matuda 38549 (LL, MO, US).

7 Tillandsia brachycaulos Schltdl., Linnaea 18: 422. 1844 [1845].

Fig. 13

Specimens eXamined: District Ixtlán, Ixtlán de Juárez, $R$. E. Schultes 662A (GH). District Juquila, San Jerónimo Coatlán, A. R. López-Ferrari, A. Espejo, J. Ceja \& A. Mendoza R. 2940 (UAMIZ). District Tuxtepec, San Juan Bautista Tuxtepec, A. R. López-Ferrari, A. Espejo \& G. Carnevali 3186 (UAMIZ); Santa María Jacatepec, C. H. Ramos \& E. Martínez S. 290 (CHAP).

8 Tillandsia butzii Mez, in Engl., Pflanzenr. IV. 32 (Heft 100, 4): 636. $1935 . \quad$ Fig. 13 Specimens examined: District Cuicatlán, Cuyamecalco Villa de Zaragoza, C. Conzatti \& I. Gómez 3491 (MEXU(x2), US). District Juchitán, San Miguel Chimalapa, C. Perret, J. Rivera H., C. Perret, H. Asborgsen, S. Solórzano, J. Avendaño, Felipe \& Elsa 110 (MEXU); Santa María Chimalapa, S. Salas M., E. Torres B., J. Rivera H., R. García S. \& G. Errerías 1377 (UAMIZ). District Juquila, San Juan Lachao A. R. López-Ferrari, A. Espejo, J. Ceja \& A. Mendoza R. 2924 (UAMIZ(X2)). District JuxtlahuacA, San Martín Peras, M. Cházaro B., M. Kimnach, R. Dorsch \& M. Negrete 6824 (MEXU, MICH, XAL); Santiago Juxtlahuaca, K. Ehlers \& R. Ehlers EM 81215 (WU according Ehlers, 2002: 55), K. Ehlers \& R. Ehlers EM 891302 (WU), K. Ehlers \& R. Ehlers EM 892303 (WU(x4)), R. Torres C. \& R. Cedillo T. 569 (MEXU). District Miahuatlán, San Jerónimo Coatlán, A. Campos V. \& R. Torres C. 1567 (MEXU), R. Torres C. \& A. Campos V. 10798 (MEXU); San Sebastián Río Hondo, J. Rzedowski 46260 (IEB, MEXU). District Mixe, San Pedro y San Pablo Ayutla, Bevan 399 (GH). District Putla, Putla Villa de Guerrero, A. GarcíaMendoza \& R. Torres C. 3117-a (MEXU). District Sola de Vega, Villa Sola de Vega, $P$. Balogh 972 (SEL), K. Ehlers \& R. Ehlers EM 79719 (WU). District Teotitlán, Santiago Texcalcingo, K. Ehlers \& R. Ehlers EM 890301 (MEXU, WU(X3)). District Tlacolula, Santo Domingo Albarradas, J. Ceja, A. Espejo, A. R. López-Ferrari \& A. Mendoza R. 1593 (UAMIZ). District Tlaxiaco, Santiago Yosondúa, J. Ceja, A. Espejo, A. R. LópezFerrari \& A. Mendoza R. 1130 (UAMIZ), J. Ceja, A. Espejo, A. R. López-Ferrari, A. Mendoza R. \& G. Carnevali 1750 (UAMIZ), K. Ehlers \& R. Ehlers EM 980408 (WU). District Tuxtepec, San Felipe Usila, P. Osorio H. 65 (MEXU). District Villa Alta, San Juan Juquila Vijanos, J. Ceja, A. Espejo, A. R. López-Ferrari, A. Mendoza R. \& G. Carnevali 1602 (UAMIZ).

9 Tillandsia califanii Rauh, Bull. Bromeliad Soc. 21: 65. 1971. Fig. 13 Specimens eXamined: District Yautepec, San Carlos Yautepec, E. Matuda 38431 $(\mathrm{MEXU}(\mathrm{x} 2))$.

10 Tillandsia callichroma L. Hrom., Bromelie 1993(1): 27. 1993. ENDEMIC.

Specimens examined: District Juquila, San Pedro Juchatengo, J. Rzedowski 19637 (ENCB(x2)); District Miahuatlán San Jerónimo Coatlán, R. Hernández M., M. Franco \& H. Susan 5149, (MEXU); San Miguel Coatlán, H. Hromadnik \& L. Hromadnik 16110 


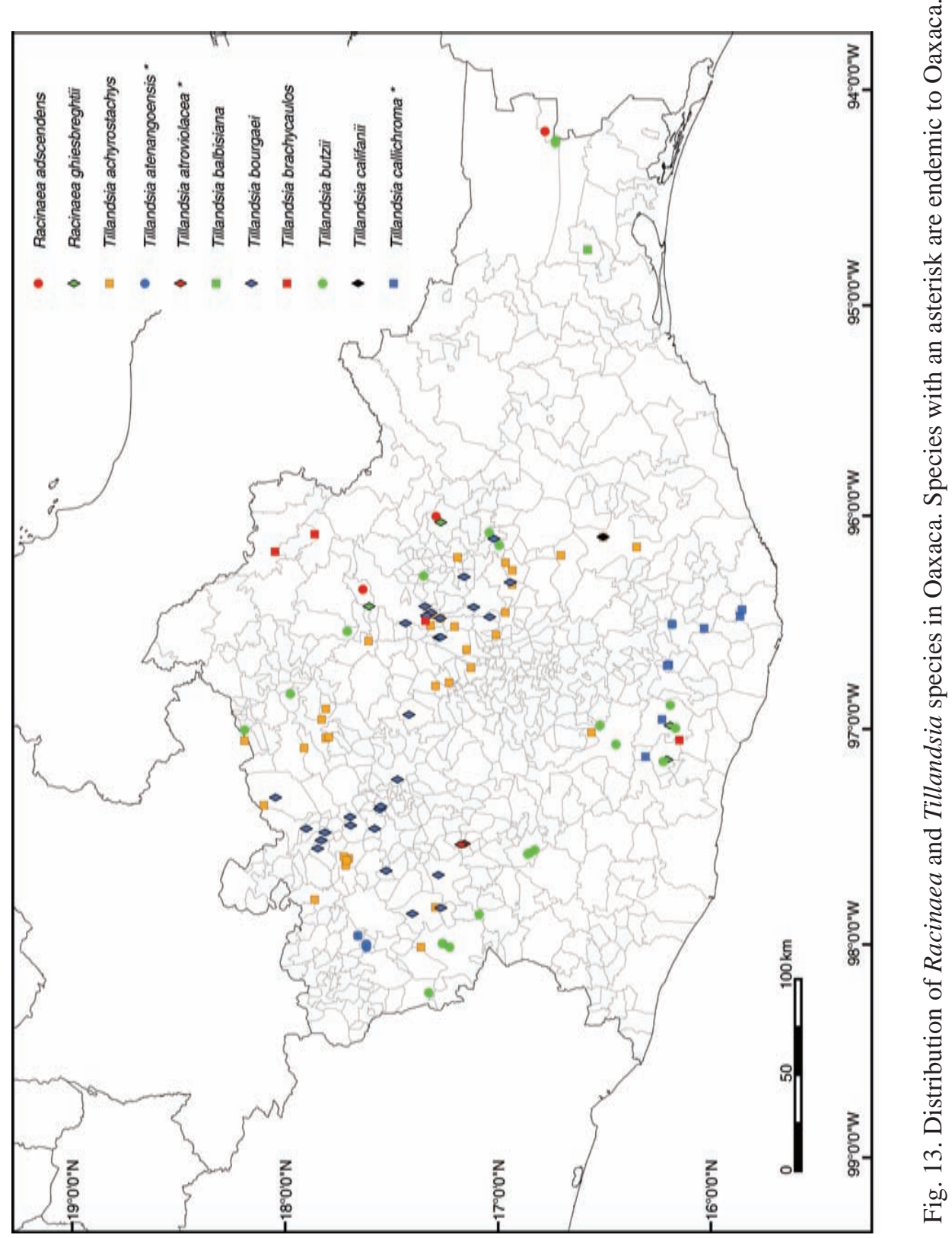


(WU(x2)), A. Reyes 1395 (CHAP); San Sebastián Río Hondo, A. R. López-Ferrari \& A. Espejo 1023 (UAMIZ). District Pochutla, San Pedro el Alto, J. Rzedowski 20647 (ENCB); San Pedro Pochutla, B. P. Reko 6277 (GH), J. Rzedowski 21188 a (ENCB, MICH(X2), US(x2)).

11 Tillandsia calothyrsus Mez, in C. DC., Monogr. Phan. 9: 704. $1896 . \quad$ Fig. 14 Specimens examined: District Cuicatlán, Santiago Nacaltepec, A. Espejo, A. R. LópezFerrari, J. Ceja, A. Mendoza R. \& I. Ramírez M. 6506 (UAMIZ(x3)), L. C. Smith 544 (GH, MEXU). District Etla, San Jerónimo Sosola, E. Guízar N. \& A. G. Miranda M. 4840 (CHAP), E. Guízar N. \& A. G. Miranda M. 4840a (CHAP, MEXU); San Juan Bautista Jayacatlán, Rusby s. n. ([S. \& D., 1979] NY). District IxTLÁN, Santa Catarina Lachatao, T. MacDougall 3-b (US), T. MacDougall 4-b (US). District NochiXtLÁn, Asunción Nochixtlán, E. Matuda 38289 (MEXU, MO, US, XAL); Magdalena Zahuatlán, B. Leuenberger \& C. Schiers 2769 (MEXU(x2)); San Miguel Huautla, A. Salinas T. \& J. SánchezKen 5697 (MEXU), A. Salinas T., G. Flores F. \& E. Martínez C. 4411-b (MEXU). District Silacayoapam, Ixpantepec Nieves, E. Guízar N. \& A. Castañeda 3742 (CHAP, MEXU, UAMIZ), E. Guízar N. \& A. Castañeda 3800 (CHAP, MEXU). District Tehuantepec, Magdalena Tequisistlán, O. van Hyning 5953 (US). District Teotitlán, Santa María Ixcatlán, P. Tenorio L. 18352 (TEX). District Tlacolula, San Lorenzo Albarradas, G. J. Martin GJM-M023 (MEXU, MO); San Pablo Villa de Mitla, T. MacDougall s. n. (US); Santo Domingo Albarradas, J. Ceja, A. Espejo, A. R. López-Ferrari \& A. Mendoza R. 1591 (UAmiz(x4)). District Tlaxiaco, Heroica Ciudad de Tlaxiaco, A. Espejo, A. R. López-Ferrari, J. Ceja \& A. Mendoza R. 6781 (UAMIZ(x2)); Santiago Yosondúa, J. Ceja, A. Espejo, A. R. López-Ferrari \& A. Mendoza R. 1108 (CICY(x3), UAMIZ(x5)), J. Ceja, A. Espejo, A. R. López-Ferrari, A. Mendoza R. \& G. Carnevali 1755. (UAMIZ(x2). Without precise locality, W. Karwinski s. n. (M(x4)), E. Matuda \& colaboradores 38538 (LL, MEXU).

12 Tillandsia capitata Griseb., Cat. pl. Cub. 255. $1866 . \quad$ Fig. 14 Specimens examined: District Pochutla, San Pedro Pochutla, C. V. Morton \& E. Makrinius 2726 (US); R. Torres C., J. L. Villaseñor, J. M. \& C A. 5253 (MEXU). District Tlaxiaco, Santiago Yosondúa, J. Ceja, A. Espejo, A. R. López-Ferrari, A. Mendoza R. \& G. Carnevali 1749 (UAMIZ).

13 Tillandsia caput-medusae E. Morren, Belgique Hort. 30: 90. 1880.

Fig. 14

Specimens examined: District Huajuapam, Santiago Cacaloxtepec, R. Torres C. \& A. García-Mendoza 6685 (MEXU, MO). District Jamiltepec, Santa Catarina Mechoacán, T. MacDougall H425 (ENCB); Santiago Pinotepa Nacional, H. Galeotti 4919B (BR, US). District Juchitán, Asunción Ixtaltepec, R. Cedillo T. 709 (MEXU, MO), C. Martínez R. 1269 (IEB, MEXU, MO), J. Meave del C. \& E. Pérez G. 1778 (MEXU(x2)), C. R. Orcutt 3300 (GH, MO, US); Ciudad Ixtepec, C. Martínez R. 1111 (MEXU), C. Martínez R. 1114 (MEXU); El Barrio de la Soledad, A. Espejo, A. R. López-Ferrari, J. Ceja, A. Mendoza R. \& I. Ramírez M. 6472 (UAMIZ); San Juan Guichicovi, R. Torres C., L. Cortés A. \& A. Campos V. 12069 (IEB, MEXU). District Pochutla, Pluma Hidalgo, A. Campos V. 2077 (CHAP, 
MEXU), A. Saynes V., S. Salas H. \& M. Elorsa C. 2130 (MEXU); San Miguel del Puerto, M. Cerón C., S. Salas M., E. Torres B. \& R. García S. 359 (UAMIZ), F. López 21 (MEXU, UAMIZ), J. Rivera H., S. Salas M. \& L. Schibli 2307 (MEXU), J. Rivera H. 2449 (MEXU), S. Salas M., A. Saynes V. \& M. Elorsa C. 4461 (MEXU), S. Salas M., A. Sánchez \& A. de Ávila 4947 (MEXU). District Tehuantepec, Magdalena Tequisistlán, C. Martínez R. 1396 (MEXU); San Pedro Huamelula, J. Rivera H., S. Salas M. \& M. Elorsa C. 2124 (MEXU); Santa María Jalapa del Marqués, R. Torres C., R. Cedillo T. \& L. Rico 512 (MEXU), R. Torres C. \& J. L. Villaseñor 5152 (MEXU, MO); Santo Domingo Tehuantepec, R. Torres C. \& J. L. Villaseñor 5137 (MEXU). District Tlacolula, San Pedro Totolapa, E. Matuda \& colaboradores 38159 (MEXU, US), R. Merrill King 2974 (TEX). District Villa Alta, Villa Hidalgo, R. E. Gereau \& A. J. Moyer 2173 (MO). District Yautepec, Nejapa de Madero, J. Ceja, A. Espejo, A. R. López-Ferrari, A. Mendoza R. \& I. Ramírez M. 1388 (UAMIZ); Santa María Ecatepec, P. Tenorio L. 18867 (MEXU).

14 Tillandsia carlos-hankii Matuda, Cact. Succ. J. (Los Angeles) 45: 186-187, f. 1-2. 1973. ENDEMIC.

Fig. 14 Specimens examined: District Cuicatlán, Santiago Nacaltepec, A. Espejo, A. R. LópezFerrari, J. Ceja, A. Mendoza R. \& I. Ramírez M. 6505 (UAMIZ(x4)). District Huajuapam, San Andrés Dinicuiti, W. Boege 3013 (MEXU(x2)), W. Boege 3015 (MEXU). District YautePec, San Carlos Yautepec, E. Matuda 38514 (CODAGEM, MEXU, UAMIZ); District ZaAchila, San Pablo Cuatro Venados, A. G. Miranda M. \& O. L. Hernández 732 (MEXU). Without precise locality, A. Lau Jr. sub D. Cathcart s. n. (SEL).

15 Tillandsia celata Ehlers \& Lautner, Bromelie 2006(1): 8-12. 2006. EndEMIC. Fig. 14 Specimens examined: Distrito Tlaxiaco, San Mateo Peñasco, K. \& R. Ehlers EM930902 (MEXU), R. Ehlers EM051902 (MEXU(x2), WU).

16 Tillandsia chaetophylla Mez, in C. DC., Monogr. Phan. 9: 726B727. 1896. Fig. 14 Specimens examined: District Cuicatlán, San Juan Bautista Cuicatlán, L. C. Smith 557 (GH). District Juquila, San Juan Lachao, R. Ehlers 8290 (WU). District Miahuatlán, San Sebastián Río Hondo, D. Cathcart s. n. (SEL). District Nochixtlán, San Miguel Huautla, A. Salinas T. \& R. Martínez S. 5980 (MEXU, XAL), A. Salinas T. \& E. Martínez C. 6285 (MEXU). District Pochutla, San Pedro Pochutla, B. P. Reko 6213 (GH). District Sola de Vega, Villa Sola de Vega, A. Espejo, A. R. López-Ferrari \& J. García-Cruz 5076 (CHAP, CICY, CIIDIR, IEB, MEXU, UAMIZ(x3)), H. Hromadnik \& L. Hromadnik 16078 (WU), C. Jurgensen 13 (FI, P), J. Rzedowski 21289 (ENCB). District Teotitlán, Teotitlán de Flores Magón, S. Schatzl 77/98 (WU). District Yautepec, San Carlos Yautepec, E. Matuda \& colaboradores 38439 (MEXU(x2), MO).

17 Tillandsia chlorophylla L. B. Sm., in Britton, N. Amer. Fl. 19: 145. $1938 . \quad$ Fig. 14 Specimens examined: District Juchitán, San Miguel Chimalapa, T. Wendt, S. Maya J. \& P. Vera 4651 (UAMIZ); Santa María Chimalapa, M. Cerón C., S. Salas M. \& H. Morales 263 (UAMIZ), M. Cerón C., S. Salas M. \& H. Morales 276 (UAMIZ), P. Tenorio L. \& T. L. Wendt 19266 (MEXU). 
18 Tillandsia circinnatioides Matuda, Cact. Succ. J. (Los Angeles) 45: 187-189, f. 4, 4a, 5. 1973.

Fig. 14

Specimens examined: District Coixtlahuaca, Tepelmeme Villa de Morelos, A. Salinas T. 7716 (MEXU(x2)). District Cuicatlán, Concepción Pápalo, A. Salinas T. \& R. Martínez S. 5927 (MEXU); San Juan Bautista Cuicatlán, C. A. Cruz Espinosa \& E. Martínez S. 425 (MEXU, UAMIZ(x2)), C. A. Cruz Espinosa \& E. San Pedro 503 (MEXU, UAMIZ(x2)), C. A. Cruz Espinosa \& E. San Pedro 1357 (MEXU), E. Martínez S. \& V. Torres H. 33543-A (MEXU), A. Salinas T., E. Martínez C. \& R. Martínez S. 6779 (MEXU); San Pedro Jaltepetongo, F. Miranda 4661 (MEXU); Santos Reyes Pápalo, R. Torres C. \& J. L. Villaseñor 5058 (MEXU); Valerio Trujano, A. García-Mendoza, R. Medina L. \& J. Sandoval 7362 (MEXU). District Etla, San Juan Bautista Atatlahuca, A. Espejo, A. R. López-Ferrari, J. Ceja, A. Mendoza R. \& I. Ramírez M. 6509 (UAMIZ). District Huajuapam, Huajuapam de León, E. Matuda s. n. (MEXU); Santo Domingo Tonalá, A. Espejo, A. R. López-Ferrari, J. Ceja, A. Mendoza R. \& G. Carnevali 6876 (UAMIZ). District Juchitán, Juchitán de Zaragoza, R. Merrill King 1614 (TEX). District JuxtlahuacA, Santos Reyes Tepejillo, J. I. Calzada 22592 (MEXU). District Teotitlán, San Antonio Nanahuatipam, J. Ceja \& A. Mendoza R. 1308 (UAMIZ), A. García-Mendoza, A. Salinas T. \& E. Martínez S. 3451 (MEXU), A. Salinas T., G. Flores F., E. Martínez C. \& S. Bravo 4518 (MEXU, XAL), A. Salinas T., J. Sánchez-Ken \& E. Martínez C. 5522 (MEXU); Teotitlán de Flores Magón, A. García-Mendoza, A. Salinas T. \& E. Martínez S. 3365 (MEXU), E. Matuda \& colaboradores 38489 (MEXU, US). District Teposcolula, Villa de Tamazulapam del Progreso, $D . H$. Lorence, A. García-Mendoza \& R. Cedillo T. 3374 (MEXU, MO). District Tlacolula, San Pedro Totolapa, E. Matuda \& colaboradores 38159 [A] (MEXU, US). Without precise locality, E. Matuda 28444 (MEXU), W. Rauh RM 15913 (US).

19 Tillandsia compressa Bertero ex Schult. \& Schult. f., Syst. veg. 7: 1210-1211. 1830.

Fig. 14

Specimens eXamined: District Ixtlán, Ixtlán de Juárez, R. García S. 782 (MEXU). DisTRICt Tuxtepec, Santa María Jacatepec, R. Torres C. \& E. Martínez S. 11035 (MEXU, MO), R. Torres C. \& L. Cortés A. 11478 (IEB, MEXU).

20 Tillandsia concolor L. B. Sm., Phytologia 7: 249, t. 1, f. 1-2. $1960 . \quad$ Fig. 14 Specimens examined: District Cuicatlán, Concepción Pápalo, A. Salinas T. \& R. Martínez S. 5935 (MEXU); San Juan Bautista Cuicatlán, A. Espejo, A. R. López-Ferrari, J. Ceja, A. Mendoza R. \& I. Ramírez M. 6516 (UAMIZ). District EtLA, San Felipe Tejalapam, J. Santana C., R. Grether \& A. Martínez B. 322 (UAMIZ). District Juchitán, Asunción Ixtaltepec, G. Carnevali, G. Campos, M. Gómez \& F. May Pat 4435 (CICY(x2)), A. GarcíaMendoza, E. Martínez S. \& C. H. Ramos 4022 (MEXU), J. Meave del C., E. Pérez G., C. Gallardo, B. Reyes \& J. Reyes 1762 (MEXU), J. Meave del C., E. Pérez G., C. Gallardo, B. Reyes \& J. Reyes 1779 (MEXU); Ciudad Ixtepec, T. MacDougall s. n. (US), C. A. Purpus 7414 (UC, US); El Barrio de la Soledad, R. Torres C., L. Cortés A. \& M. L. Torres C. 9727 (IEB, MEXU); Juchitán de Zaragoza, T. MacDougall s. n. (US); San Blas Atempa, $R$. Merrill King 1385 (TEX, US); San Juan Guichicovi, W. G. Williams Jr. \& D. Francoeur 42 (MICH); Santa María Xadani, R. Merrill King 1556 (TEX, US); Santiago Niltepec, 
R. Merrill King 1797 (TEX). District Pochutla, San Miguel del Puerto, S. Salas M., J. Rivera H., R. García S., J. Meave del C. \& M. Elorsa 2049 (SERO). District TehuAntepec, Salina Cruz, R. Merrill King 1295 (TEX, US); San Pedro Huamelula, J. Rivera H. 878 (UAMIZ); Santo Domingo Tehuantepec, M. B. Foster \& O. Van Hyning 2941 (US), R. Merrill King 1336 (MICH, TEX, US), M. L. Torres C., R. Torres C., P. Tenorio L. \& C. Martínez 328 (IEB, MEXU, MO), M. L. Torres C., R. Torres C., O. Téllez \& C. Martínez 387 (MEXU, MO), M. L. Torres C., R. Torres C., L. Cortés A. \& C. Martínez 906 (IEB, MEXU, MO, XAL). District Tlacolula, San Pedro Totolapa, $R$. Merrill King 2973 (TEX(x2)), R. Torres C., L. Cortés A. \& C. Martínez 9876 (MEXU), J. Utley \& K. Burt-Utley 6795 (MEXU). District Yautepec, San Carlos Yautepec, J. Ceja, A. Espejo, A. R. López-Ferrari, A. Mendoza R. \& I. Ramírez M. 1383 (UAMIZ), E. Matuda \& colaboradores 38498 (MEXU, US), A. Salinas T. \& E. Martínez C. 8156 (MEXU), A. Salinas T. \& E. Martínez C. 8168 (MEXU); Santa María Ecatepec, A. Salinas T. \& E. Martínez C. 8231 (IEB, MEXU).

21 Tillandsia copalaensis Ehlers, Bromelie 2001(3): 64-66, figs. 2002. ENDEMIC. Fig. 14 Specimens examined: District Juxtlahuaca, Santiago Juxtlahuaca, K. Ehlers \& R. Ehlers EM 8178 (MEXU, WU), R. Ehlers \& K. Ehlers EM 78127 (WU), R. Ehlers \& K. Ehlers EM 892212 (WU). District Putla, Putla Villa de Guerrero, J. Rutschmann s. n. (WU).

22 Tillandsia delicata Ehlers, Bromelie 1999(3): 64-70, figs. 2000. EndEMIC. Fig. 14 Specimens eXamined: District Juquila, San Gabriel Mixtepec, J. Lautner s. n. (WU); San Juan Lachao, K. Ehlers \& R. Ehlers EM 8316 ([Bromelie 1999(3) WU]), K. Ehlers \& R. Ehlers EM 881501 ([Bromelie 1999(3) MEXU, WU]), G. Noller 20 (WU).

23 Tillandsia dugesii Baker, J. Bot. 25: 278. 1887.

Fig. 15 Specimens examined: District Etla, Santiago Tenango, M. B. Foster \& O. Van Hyning 2988 (US). District Juxtlahuaca, San Juan Mixtepec, A. García-Mendoza \& J. Reyes 5035 (MEXU). District Teposcolula, San Pedro y San Pablo Teposcolula, A. García-Mendoza, D. H. Lorence \& D. Frame 1697 (MEXU). District Nochixtlán, Santo Domingo Yanhuitlán, A. Espejo, A. R. López-Ferrari, J. Ceja, A. Mendoza R. \& G. Carnevali 6848 (UAMIZ(x2)), A. Espejo, A. R. López-Ferrari, J. Ceja, A. Mendoza R. \& G. Carnevali 6849 (UAMIZ(x2)). District Tlaxiaco, Heroica Ciudad de Tlaxiaco, J. Ceja \& A. Mendoza R. 1624 (UAMIZ(x4)); San Pedro Molinos, A. Espejo, A. R. López-Ferrari, J. Ceja \& A. Mendoza R. 6263 (CICY(x3), UAMIZ(x5)), A. Espejo, A. R. López-Ferrari, J. Ceja \& A. Mendoza R. 6263A (UAMIZ(x5)). District ZaAchila, San Pablo Cuatro Venados, A. G. Miranda M. 830 (CHAP). Without precise locality, E. Matuda \& colaboradores 38547 (LL, US).

24 Tillandsia fasciculata Sw., Prodr. 56. 1788.

Fig. 15 Specimens examined: District Cuicatlán, San Juan Bautista Cuicatlán, A. Salinas T., P. Dávila A., R. Medina L., P. Tenorio L. \& A. Ramírez R. 5422-a (MEXU); Valerio Trujano, without collector 5 (MEXU), without collector 6 (MEXU). District EjuTLA, La Compañía, J. Ceja, A. Espejo, A. R. López-Ferrari \& A. Mendoza R. 1265 (UAMIZ(x2)). District Etla, San Jerónimo Sosola, A. Salinas T., E. Martínez C. \& R. Martínez S. 6873 (MEXU). District Huajuapam, San Andrés Dinicuiti, E. Matuda s. n. (MEXU). District Ixtlán, 


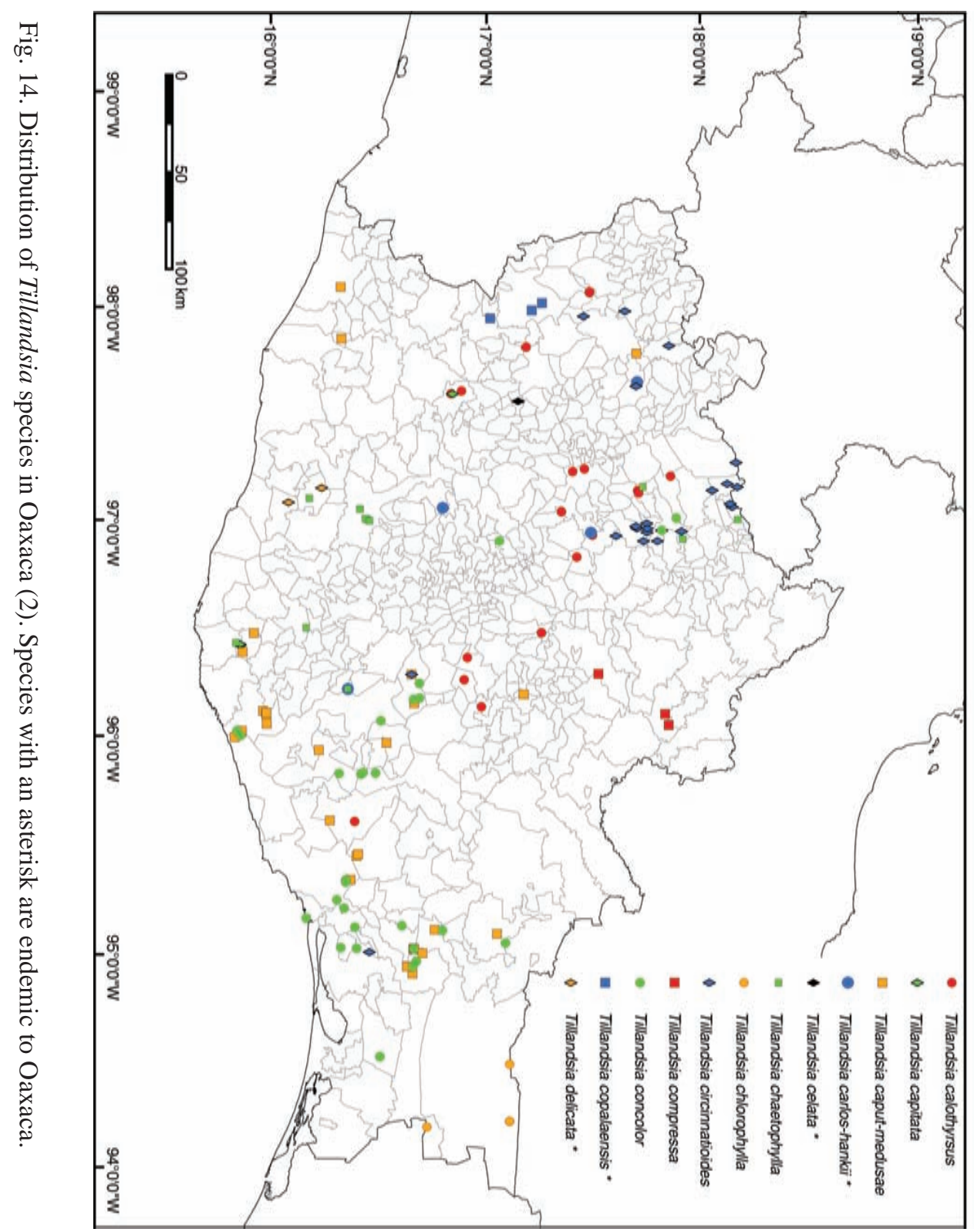


Santiago Comaltepec, W. Rauh 16098 (US). District Juchitán, Asunción Ixtaltepec, A. Espejo, A. R. López-Ferrari, J. Ceja, A. Mendoza R. \& I. Ramírez M. 6487 (UAMIZ), R. Torres C. \& C. Martínez 6091 (MEXU, MO), R. Torres C. \& E. Martínez S. 11207 (MEXU), R. Torres C., L. Cortés A. \& A. Campos V. 11981 (MEXU), W. G. Williams Jr. \& D. Francoeur 35 (MICH); San Miguel Chimalapa, R. Torres C. \& C. Martínez 10619 (MEXU); Santa María Chimalapa, L. M. González-Villareal, R. Ramírez D., R. González T. \& R. González A. 3795 (IBUG). District Juxtlahuaca, San Juan Mixtepec, A. García-Mendoza, A. Campos V. \& J. Reyes 3638 (MEXU). District Miahuatlán, Miahuatlán de Porfirio Díaz, $R$. Torres C., D. Neil \& F. Neil 2206 (MEXU); San Jerónimo Coatlán, A. García-Mendoza, A. Campos V. \& J. Reyes 4665 (MEXU); San Juan Mixtepec, E. Hunn 1901 (MEXU). District Pochutla, Pluma Hidalgo, A. Espejo, A. R. López-Ferrari, J. Ceja \& A. Mendoza R. 6315 (UAMIZ(x2)); San Pedro Pochutla, E. Makrinius 536 (US), R. Torres C., J. L. Villaseñor, J. M. \& C. A. 5255 (MEXU). District Putla, Putla Villa de Guerrero, P. Tenorio L., R. Torres C. \& C. Romero de T. 3605 (MEXU), R. Torres C. \& A. García-Mendoza 6703 (MEXU, MO). District Sola de Vega, Santa María Sola, V. García G. 12 (UAMIZ(x3)); Villa Sola de Vega, V. Flores R. s. n. (CHAP, UAMIZ), D. E. Breedlove 9846 ([S. \& D., 1979] DS). District Tehuantepec, San Miguel Tenango, C. Martínez R. 1425 (MEXU); Santo Domingo Tehuantepec, M. B. Foster \& O. Van Hyning 2961 (US), T. MacDougall 4 (MEXU, US), C. Martínez R. 442 (MEXU), J. N. Rose \& W. Hough 5865 (GH), M. L. Torres C., R. Torres C., C. Martínez \& J. Martínez 827 (MEXU). District Teotitlán, Teotitlán de Flores Magón, R. Torres C. \& M. A. Martínez 6632 (IEB, MEXU), R. Torres C. \& J. L. Villaseñor 5050 (MEXU). District Teposcolula, Santa María Chilapa de Díaz, J. I. Calzada 23763 (MEXU). District Tlacolula, San Jerónimo Tlacochahuaya, C. Conzatti 3953 (MEXU(x2)); San Pablo Villa de Mitla R. Torres C., D. H. Lorence \& A. GarcíaMendoza 3974 (MEXU, XAL); Teotitlán del Valle, L. A. Kenoyer 1624 (GH); Tlacolula de Matamoros, J. Ceja \& A. Mendoza R. 1206 (UAMIZ(x2)), A. Espejo \& A. R. López-Ferrari 6760 (UAmiZ(x2)). District Tlaxiaco, Heroica Ciudad de Tlaxiaco, A. Espejo, A. $R$. López-Ferrari, J. Ceja \& A. Mendoza R. 6766 (UAMIZ(x2)); Santiago Yosondúa, J. Ceja, A. Espejo, A. R. López-Ferrari, A. Mendoza R. \& G. Carnevali 1761 (UAMIZ(x2)). Without precise locality, A. Lau Jr. sub H. E. Luther s. n. (SEL).

25 Tillandsia festucoides Brongn. ex Mez, in C. DC., Monogr. Phan. 9: 678-679. 1896.

Fig. 15

Specimens examined: District Tehuantepec, Santa María Guienagati, P. Tenorio L., $R$. Torres C. \& C. Martínez 11168 (MEXU(x2)).

26 Tillandsia filifolia Schltdl. \& Cham., Linnaea 6: 53. 1831.

Fig. 15

Specimens examined: District Cuicatlán, San Juan Bautista Cuicatlán, C. Conzatti 3557 (MEXU(x2), US). District Choapam, San Juan Lalana, R. E. Schultes \& B. P. Reko 803 (GH). District Ixtlán, Santiago Comaltepec, R. E. Gereau \& G. J. Martin 1984 (MEXU, MO), W. L. Graham 1406 (MICH). District Juchitán, Matías Romero, L. Williams 9096 (GH); Santa María Chimalapa, T. MacDougall s. n. (US), E. Torres B., J. Rivera H., T. Álvarez R. \& D. Acuca V. 1361 (SERO, UAMIZ), T. Wendt, A. Villalobos C. \& I. Navarrete 4006 (MEXU). District Teotitlán, San Bartolomé Ayautla, R. Torres C., R. Cedillo T. \& 
L. Cortés A. 831 (MEXU). District Tuxtepec, San Felipe Usila, R. E. Schultes \& B. P. Reko 672 (GH); San Pedro Ixcatlán, J. I. Calzada 10257 (XAL).

27 Tillandsia flabellata Baker, J. Bot. 25: 242. 1887.

Fig. 15

Specimens examined: District Juchitán, Asunción Ixtaltepec S. Salas M., M. Elorsa C. \& A. Saynes V. 4472 (UAMIZ); San Miguel Chimalapa S. Maya J. 1228 (MEXU, UAMIZ). District Putla, Putla Villa de Guerrero, A. García-Mendoza, R. Torres C. \& L. Cortés A. $3138(\mathrm{MEXU}(\mathrm{x} 2))$.

28 Tillandsia flavobracteata Matuda, Cact. Suc. Mex. 20: 97-99, f. 49-50. $1975 . \quad$ Fig. 15 Specimens examined: District Juchitán, Santa María Chimalapa, H. Hernández G. 756 (UAMIZ). District Tuxtepec, San Juan Bautista Tuxtepec, A. R. López-Ferrari, A. Espejo \& G. Carnevali 3185 (UAMIZ(x3)).

29 Tillandsia foliosa M. Martens \& Galeotti, Bull. Acad. Roy. Sci. Bruxelles 10: 119-120. 1843.

Specimens examined: District Tuxtepec, San Felipe Usila, R. E. Schultes \& B. P. Reko 664 $(\mathrm{GH})$.

30 Tillandsia fuchsii W. Till, Bromelie 1990(2): 30-32, f. a-g. 1990.

Fig. 15 Specimens examined: District Putla, Putla Villa de Guerrero, $R$. Ehlers \& K. Ehlers s. $n$. (WU). District Tehuantepec, Magdalena Tequisistlán, O. van Hyning 5952 (US). District Tlacolula, San Pedro Totolapa, M. B. Foster \& O. Van Hyning 2930 (US); Santo Domingo Albarradas, J. Ceja, A. Espejo, A. R. López-Ferrari \& A. Mendoza R. 1585 (UAMIZ). District Yautepec, Nejapa de Madero, A. Espejo, A. R. López-Ferrari, J. Ceja, A. Mendoza R. \& I. Ramírez M. 1387 (UAMIZ); San Juan Lajarcia, E. W. Nelson 2561 (GH, US), S. Schatzl 75/23 (WU), E. Zecher s. n. (WU).

31 Tillandsia glabrior (L. B. Sm.) López-Ferrari, Espejo \& I. Ramírez, Selbyana 25: 33-86. ENDEMIC.

Fig. 15

Specimens examined: District Tehuantepec, Magdalena Tequisistlán, M. B. Foster \& O. Van Hyning 2937 (US), O. van Hyning 5944 (US). District Tlacolula, Santa María Zoquitlán, S. Acosta C. 929 (UAMIZ). Without precise locality, T. MacDougall 5 sub E. Matuda 26049 (US).

32 Tillandsia grandis Schltdl., Linnaea 18: 424-426. 1844 [1845].

Fig. 15

Specimens examined: District Coixtlahuaca, Tepelmeme Villa de Morelos, A. Salinas T. \& E. L. Cruz P. 7836 (MEXU). District Cuicatlán, San Juan Bautista Cuicatlán, A. Salinas T., E. Martínez C. \& R. Martínez S. 6671 (MEXU); Santiago Nacaltepec, W. R. Ernst 2487 (MEXU(x2), US(x2)), E. Martínez S. \& V. Torres H. 33479 (MEXU(x2)). DisTrict EtLA, San Juan Bautista Atatlahuca, C. Delgadillo M. 217 (MEXU(x2), MO, XAL). District Ixtlán, Abejones, R. Medina L., L. Alvarado C. \& J. Sandoval 1045 (MEXU). District Juchitán, San Miguel Chimalapa, T. MacDougall s. n. (GH). District TehuanTEPEC, Guevea de Humboldt, A. Campos V. \& R. Torres C. 3614 (IEB, MEXU), R. Torres 
C. \& L. Cortés A. 14360 (GH, IEB, MEXU). District Teotitlán, Huautla de Jiménez, X. Munn \& T. Kasey 1182 (MEXU); Santa María Chilchotla, E. Matuda 38623 (LL, MEXU). District Villa Alta, San Andrés Yaá, R. Cedillo T. \& R. Torres C. 1201 (ENCB, MEXU, MO), E. Ramírez G. \& P. Ramírez C. 552 (IBUG, IEB, MEXU).

33 Tillandsia guatemalensis L. B. Sm., Contr. U. S. Natl. Herb. 29: 281. $1949 . \quad$ Fig. 15 Specimens examined: District Etla, San Juan del Estado, G. J. Martin GJM-M261 (MEXU, MO). District Juchitán, San Miguel Chimalapa, M. Ishiki I. 1289 (IEB, MO), M. Ishiki I. 1383 (MEXU(x2)), T. MacDougall s. n. (US(x2)), R. Torres C. \& C. Martínez 4861 (MEXU, MO); Santo Domingo Zanatepec, R. Torres C. \& C. Martínez 4837 (MEXU). District Miahuatlán, San Jerónimo Coatlán, R. Torres C. \& A. Campos V. 10876 (MEXU).

34 Tillandsia gymnobotrya Baker, J. Bot. 25: 243-244. 1887.

Fig. 15

Specimens examined: District Coixtlahuaca, Tepelmeme Villa de Morelos, $R$. Cruz C. 2339 (ENCB). District Cuicatlán, San Pedro Jocotipac, A. Salinas T., G. Flores F. \& E. Martínez C. 4392 (MEXU), A. Salinas T. \& J. Sánchez-Ken 5682 (MEXU); Santiago Nacaltepec, A. Salinas T. \& V. Juárez J. 7046 (MEXU). District IxtLán, Capulalpam de Méndez, S. Figueroa B. \& F. Y. Guzmán R. 337 (CHAP), S. Figueroa B. \& F. Y. Guzmán R. 362 (UAMIZ(x3)); Ixtlán de Juárez, A. Espejo, A. R. López-Ferrari, J. Ceja, A. Mendoza R. \& G. Carnevali 6697 (UAMIZ(x2)), J. Rivera H., I. Sánchez G. \& J. C. Flores V. 2065 (MEXU), R. Torres C. \& L. Cortés A. 9364 (MEXU); Santa Catarina Lachatao, $H$. H. Iltis 27141 (MEXU), T. MacDougall s. n. (US); Santiago Comaltepec, R. Cedillo T., D. H. Lorence \& G. Martin 657 (MEXU), T. MacDougall 854 (US). District MiahuatLÁn, San Jerónimo Coatlán, A. García-Mendoza, A. Campos V. \& J. Reyes 4606 (MEXU). District Mixe, Totontepec Villa de Morelos, A. García-Mendoza \& R. Torres C. 2036 (MEXU, MO), J. Rivera R. \& G. J. Martin 1451 (MEXU), P. Tenorio L. \& R. Torres C. 11060 (MEXU). District Nochixtlán, San Miguel Chicahua, A. García-Mendoza, F. Martínez \& F. Mérida 2081 (F, MEXU). District Teotitlán, Mazatlán Villa de Flores, M. Cruz D. s. $n$. (XAL(x2)), X. Munn \& F. Mendoza 1660 (MEXU); San Jerónimo Tecoatl, P. Tenorio L. \& F. Tenorio L. 18578 (MEXU), R. Torres C. \& J. L. Villaseñor 5044 (MEXU); San Lucas Zoquiapam, R. E. Schultes \& B. P. Reko 413 (GH); Teotitlán de Flores Magón, A. Salinas T. \& E. Martínez C. 8132 (MEXU), C. E. Smith Jr., F. A. Peterson \& N. Tejeda 4182 (MEXU, US), P. Tenorio L. 20033 (MEXU), R. Torres C. \& M. A. Martínez 6512 (MEXU), without colector 583 (SEL). District Teposcolula, Villa Tejupam de la Unión, A. Espejo, A. R. López-Ferrari, J. Ceja \& A. Mendoza R. 6229 (IEB, UAMIZ(x3)), A. García-Mendoza, R. Torres C. \& L. Cortés A. 3089 (MEXU(x2)). District Tlacolula, Santo Domingo Albarradas, J. Ceja, A. Espejo, A. R. López-Ferrari \& A. Mendoza R. 1597 (UAMIZ). District Tuxtepec, San Felipe Usila, A. Rincón G. \& C. Gallardo H. 426 (MEXU), A. Rincón G., C. Gallardo H. \& R. Wong 498 (MEXU), A. Rincón G. \& C. Gallardo H. 502 (MEXU). District ViLla Alta, San Francisco Cajonos, H. H. Iltis 27143 (MEXU); Santa María Yalina, J. Ceja, A. Espejo, A. R. López-Ferrari, A. Mendoza R. \& G. Carnevali 1606 (UAMIZ); Tanetze de Zaragoza, X. Munn, R. del Castillo, R. Rivera \& N. Sánchez 252 (MEXU). Without precise locality, E. Matuda \& colaboradores 38541 (LL, MEXU(x2), US). 


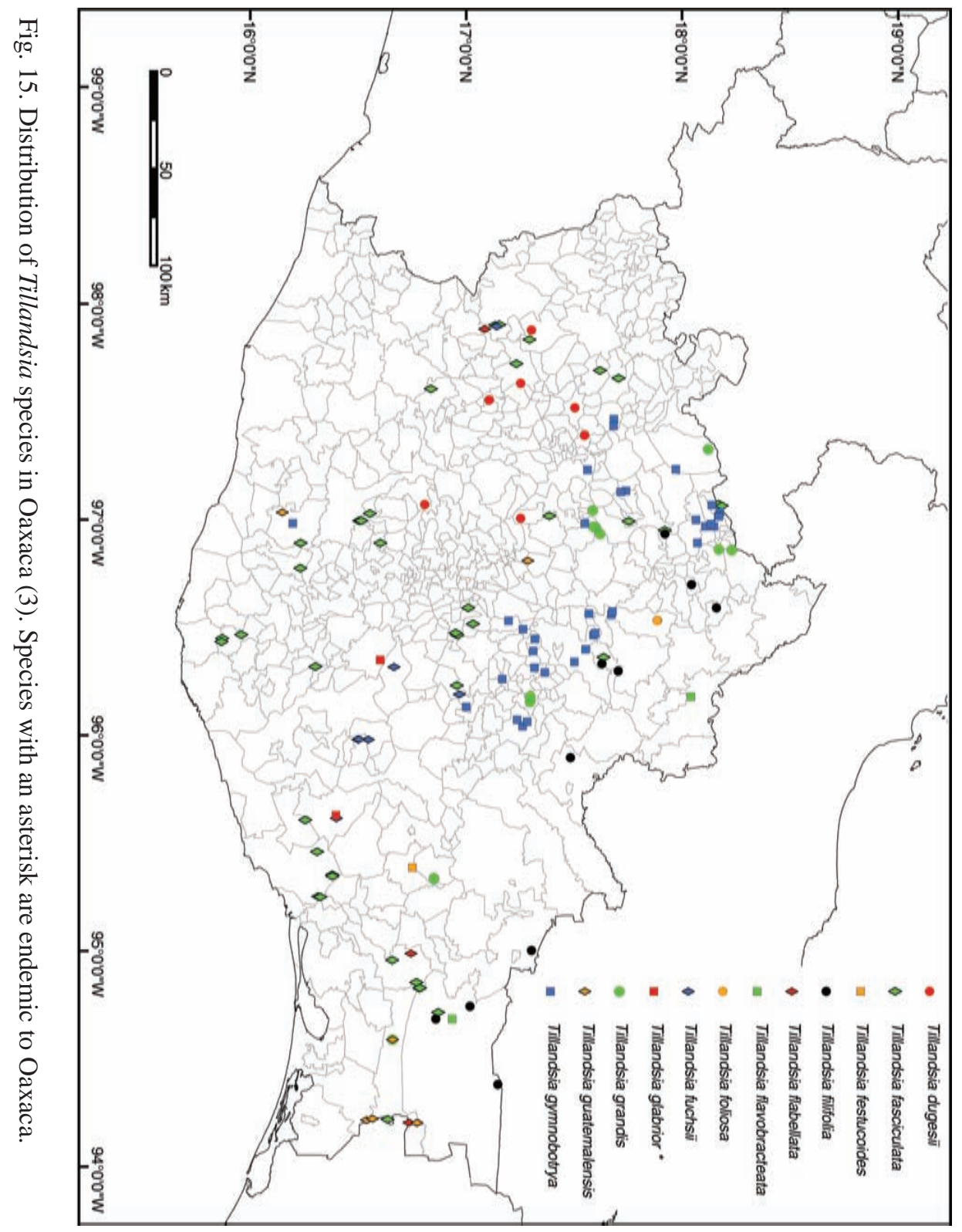


35 Tillandsia hammeri Rauh \& Ehlers, in Rauh, Trop. Subtrop. Pflanzenw. 60: 67-69 (969971), f. 46. 1987.

Specimens examined: District Cuicatlán, San Juan Bautista Cuicatlán, J. Calónico S., J. P. Abascal \& C. A. Cruz-Espinosa 23971 (MEXU), C. A. Cruz Espinosa \& E. San Pedro 488 (MEXU, UAMIZ(x2)), C. A. Cruz Espinosa \& E. San Pedro 1018 (MEXU), E. Martínez S. \& V. Torres H. 33549 (MEXU), R. Medina L., L. Alvarado C. \& J. Sandoval 1039 (MEXU), F. Miranda 4699 (MEXU), A. Salinas T. \& E. Martínez C. 8113 (MEXU), without colector 4 (MEXU); San Pedro Jaltepetongo, C. Conzatti 1743 (MEXU, US), C. Conzatti s. n. ([S. \& D., 1979] US); San Pedro Jocotipac, A. Salinas T. \& A. Reyes G. 4860 (MEXU, TEX); Santiago Nacaltepec, C. A. Cruz Espinosa \& E. Martínez S. 336 (MEXU, UAMIZ(x2)), R. Ehlers \& K. Ehlers EM 850501 (WU(x2)). Distric EtLA, San Jerónimo Sosola, A. Salinas T., E. Martínez C. \& R. Martínez S. 6633 (MEXU); San Juan Bautista Atatlahuca, A. Espejo, A. R. López-Ferrari, J. Ceja, A. Mendoza R. \& I. Ramírez M. 6508 (UAMIZ), G. Hammer s. n. (HEID), P. Koide s. n. (SEL). District Tehuantepec, Santa María Jalapa del Marqués, C. Martínez R. 445 (MEXU); Santo Domingo Tehuantepec, M. L. Torres C., R. Torres C. \& C. Martínez 212 (MEXU, MO), R. Torres C. \& C. Martínez 8291 (MEXU). District Teotitlán, Santa María Ixcatlán, P. Tenorio L. \& E. Martínez C. 17405 (MEXU).

36 Tillandsia huamelulaensis Ehlers, J. Bromeliad Soc. 56: 57-59, fig. 4-6. 2006, "huamenulaensis".

Specimens examined: District Juchitán, Asunción Ixtaltepec, J. Meave del C. \& E. Pérez G. 1866 (MEXU), R. Merrill King 630 (MEXU, TEX), R. Torres C. \& E. Martínez S. 11208 (MEXU), R. Torres C. \& E. Martínez S. 11225 (IEB), R. Torres C., L. Cortés A. \& A. Campos V. 11982 (MEXU), W. G. Williams Jr. \& D. Francoeur 21 (US); Ciudad Ixtepec, C. Martínez R. 1110 (MEXU, MO), C. Martínez R. 1115 (MEXU), R. Merrill King 1492 (TEX); Juchitán de Zaragoza, R. Merrill King 360 (TEX), R. Merrill King 1632 (TEX); San Blas Atempa, R. Merrill King 1389 (TEX). District Juquila, San Pedro Mixtepec, W. R. Ernst 2839 (MEXU, MICH, US), C. S. Gardner 250 (US). District Pochutla, Santa María Huatulco, S. Salas M., M. Elorsa C. \& L. Schibli 3805 (UAMIZ(x2)). District Tehuantepec, San Pedro Huamelula, $R$. Ehlers EM030406 (MEXU(x6)); Santa María Jalapa del Marqués, R. Torres C. \& J. L. Villaseñor 5145 (MEXU); Santiago Laollaga, R. Torres C. \& C. Martínez 5841 (MEXU, MO), R. Torres C., L. Cortés A. \& M. P. Ramírez 9088 (MEXU); Santo Domingo Tehuantepec, C. Martínez R. 64 (FCME), R. Merrill King 1352 (TEX). DisTrict Tlacolula, San Pedro Totolapa, R. Merrill King 2975 (TEX).

37 Tillandsia imperialis E. Morren ex Roezl, Deutsche Gärtn.-Zeitung 118, 1881. Fig. 16 Specimens examined: District Ixtlán, Ixtlán de Juárez, A. Espejo, A. R. López - Ferrari, J. Ceja, A. Mendoza R. \& G. Carnevali 6686 (UAMIZ), D. H. Lorence, G. Lorence \& R. Torres C. 4022 (MEXU); Santiago Comaltepec, R. Cedillo T., D. H. Lorence \& G. Martin 665 (MEXU). District Juchitán, San Miguel Chimalapa, R. Torres C. \& C. Martínez 4855 (MEXU). District Mixe, San Pedro y San Pablo Ayutla, G. J. Martin GJM-M212 (MEXU, MO); Totontepec Villa de Morelos, A. García-Mendoza \& R. Torres C. 2028 
(MEXU), R. E. Gereau, A. Flores M.\& G. I. Manzanero M. 2088 (MEXU, MO), J. Rivera R. \& G. J. Martin 1454 (MEXU), R. Torres C. \& C. Martínez 4983 (MEXU, MO), R. Torres C. \& C. Martínez 9068 (MEXU). Distric Teotitlán, Mazatlán Villa de Flores, X. Munn \& F. Mendoza 1657 (MEXU); San Lucas Zoquiapam, R. E. Schultes \& B. P. Reko 414 (GH); Teotitlán de Flores Magón, C. E. Smith Jr., F. A. Peterson \& N. Tejeda 4198 (GH, MEXU). District Villa Alta, San Andrés Yaá, R. Torres C. \& E. Ramírez 8549 (IEB, MEXU, MO); San Ildefonso Villa Alta, B. P. Reko 4013 ([S. \& D., 1979] US).

38 Tillandsia ionantha Planch., Fl. Serres 10: 101, t. 1006. 1855. Fig. 16 Specimens examined: District Cuicatlán, San Juan Bautista Cuicatlán, C. A. Cruz Espinosa \& E. San Pedro 487 (IEB, MEXU), L. Hardison, S. Sundberg, M. Lavin, A. Whittemore \& K. Bear 99 (TEX), E. Martínez S. \& V. Torres H. 33548 (MEXU), R. Medina L., L. Alvarado C. \& J. Sandoval 1040 (MEXU), S. Salas M. 2069 (MEXU), A. Salinas T., P. Dávila A., R. Medina L., P. Tenorio L. \& A. Ramírez R. 5420 (MEXU), A. Salinas T. \& E. Martínez C. 8110 (MEXU); San Pedro Jaltepetongo, C. Conzatti 1744 ([S. \& D., 1979] US). District Huajuapam, San Pedro y San Pablo Tequixtepec, $R$. Torres C. \& J. L. Villaseñor 5004 (MEXU, MO). District Juchitán, Asunción Ixtaltepec, D. H. Lorence \& R. Cedillo T. 3094 (MEXU); El Barrio de la Soledad, R. Torres C. \& E. Cabrera 6185 (MEXU(x2), MO); San Francisco del Mar, K. Fabián Martínez, C. Vásquez \& M. Pérez 138 (UAMIZ). District Juxtlahuaca, Santos Reyes Tepejillo, J. I. Calzada 22600 (MEXU). District Miahuatlán, San Juan Mixtepec, E. Hunn OAX-927 (MEXU(2)). District Tehuantepec, Magdalena Tequisistlán, C. Martínez R. 1397 (FCME, MEXU); San Mateo del Mar, D. Zizumbo V. \& P. Colunga G. M. 508 (MEXU); San Pedro Huamelula, J. Lautner s. n. (WU), R. Wulfinghoff s. n. (WU(x2)); Santo Domingo Tehuantepec, M. L. Torres C., R. Torres C. \& C. Martínez 116 (MEXU, MO), M. L. Torres C., R. Torres C. \& C. Martínez 705 (MEXU), M. L. Torres C., R. Torres C., C. Martínez \& J. Martínez 838 (MEXU), R. Torres C. 4228 (MEXU), R. Torres C. \& C. Martínez 8294 (MEXU). District Teotitlán, Santa María Ixcatlán, P. Tenorio L. \& E. Martínez C. 17398 (MEXU). District Tlacolula, San Pedro Quiatoni, A. Flores M. 1248 (MEXU, UAMIZ). District Tlaxiaco, Santiago Yosondúa, J. Ceja, A. Espejo, A. R. López-Ferrari, A. Mendoza R. \& G. Carnevali 1763 (UAMIZ). District Yautepec, Nejapa de Madero, P. Koide \& W. Schuster s. n. (MEXU, SEL, US). Without precise locality, L. H. Bailey 565 (GH), D. Barry 40 ([S. \& D., 1979] US).

39 Tillandsia juncea (Ruiz \& Pav.) Poir., in Lam., Encycl. Suppl. 5: 309. $1817 . \quad$ Fig. 16 Specimens examined: District Cuicatlán, San Juan Bautista Cuicatlán, L. C. Smith 551 (GH). District Ixtlán, Capulalpam de Méndez, S. Figueroa B. \& F. Y. Guzmán R. 388 (UAMIZ); Ixtlán de Juárez, J. García R. 218 (UAMIZ); San Juan Chicomezúchil, A. R. López-Ferrari, A. Espejo, J. Ceja, A. Mendoza R. \& G. Carnevali 3078 (UAMIZ). District Juchitán, El Barrio de la Soledad, W. Seaborn 1973 (US); Juchitán de Zaragoza, M. B. Foster \& O. Van Hyning 2964 (US); San Miguel Chimalapa, R. Torres C. \& C. Martínez 10620 (MEXU). District Juquila, San Pedro Juchatengo, J. Rzedowski 19631 (ENCB). District Juxtlahuaca, San Juan Mixtepec, J. Reyes S. 146 (MEXU), J. Reyes S. 241 (MEXU), J. Reyes S. 1261 (MEXU); San Sebastián Tecomaxtlahuaca, G. Ocampo \& S. Zamudio R. 745 (IEB, UAMIZ); Santiago Juxtlahuaca, R. Torres C., D. Neil \& F. Neil 
2179 (ENCB, MEXU, MO). District Mixe, Totontepec Villa de Morelos, J. Rivera R. JR1091 (MEXU(x2)). District Pochutla, San Pedro el Alto, A. Espejo, A. R. López-Ferrari, J. Ceja \& A. Mendoza R. 6327 (UAMIZ); San Pedro Pochutla, E. Makrinius 563 (US). District Putla, Putla Villa de Guerrero, S. D. Koch \& M. González L. 86108 (CIIDIR, ENCB), R. Torres C., A. García-Mendoza \& L. Cortés A. 9625 (MEXU). District Sola de Vega, San Ildefonso Sola, J. Ceja, A. Espejo, A. R. López-Ferrari \& A. Mendoza R. 1267 (UAMIZ). District Tehuantepec, Santiago Lachiguiri, A. Campos V. \& R. Torres C. 3676 a (FCME, MEXU, MO), R. Torres C. \& A. Campos V. 13906 (MO); Santo Domingo Tehuantepec, M. L. Torres C., R. Torres C., L. Cortés A. \& C. Martínez 905 (IEB, MEXU). District Teotitlán, Teotitlán de Flores Magón, F. González Medrano, P. Dávila, J. L. Villaseñor R. \& P. Ruíz F-834 (MEXU). District Teposcolula, Villa de Tamazulapam del Progreso, J. I. Calzada 23936 (MEXU), A. García-Mendoza, R. Torres C. \& L. Cortés A. 3108 (MEXU), D. H. Lorence, A. García-Mendoza \& R. Cedillo T. 3381 (MEXU), J. Rzedowski 19701 (ENCB, MEXU, MICH), R. Torres C., A. García-Mendoza \& L. Cortés A. 9590 (MEXU). District Tlacolula, Santo Domingo Albarradas, J. Ceja, A. Espejo, A. R. López-Ferrari \& A. Mendoza R. 1583 (UAMIZ), J. Ceja, A. Espejo, A. R. López-Ferrari \& A. Mendoza R. 1599 (UAMIZ). District Tlaxiaco, Heroica Ciudad de Tlaxiaco, A. Espejo, A. R. López-Ferrari, J. Ceja \& A. Mendoza R. 6775 (UAMIZ); San Agustín Tlacotepec, J. Ceja, A. Espejo, A. R. López-Ferrari, A. Mendoza R. \& G. Carnevali 1747 (UAMIZ(x2)). District Tuxtepec, Acatlán de Pérez Figueroa, E. Guízar N. \& J. C. Echevarría 5701 (CHAP, IEB, UAMIZ). District Villa Alta, Santiago Zoochila, R. Torres C., P. Tenorio L. \& C. Romero de T. 2911 (ENCB, MEXU). District Yautepec, San Carlos Yautepec, A. Flores M. 1203 (MEXU, UAMIZ), E. Matuda \& colaboradores 38542 (MEXU); Santa María Ecatepec R. Torres C. \& C. Martínez 5655 (IEB, MEXU, MO). Without precise locality, H. Galeotti 4917 (BR).

40 Tillandsia kalmbacheri Matuda, Cact. Suc. Mex. 19: 26, f. 16. 1974.

Fig. 16 Specimens eXamined: District Juquila, San Juan Lachao, J. Ceja, A. Espejo, A. R. LópezFerrari \& A. Mendoza R. 1283 (CICY(x2), UAMIZ(x5)). District Miahuatlán, San Jerónimo Coatlán, R. Hernández M., M. Franco \& H. Susan 5242 (ENCB, MEXU(x2), MO). District Yautepec, Santa María Ecatepec, T. MacDougall 5 (MEXU).

41 Tillandsia kirchhoffiana Wittm., Gartenflora 38: 107-109, f. 22. 1889.

Fig. 16

Specimens eXAmined: District Ixtlán, Ixtlán de Juárez, J. Rzedowski 33783 (ENCB(x2)). District Tehuantepec, Guevea de Humboldt, A. Campos V. \& R. Torres C. 3591 (MEXU, $\mathrm{MO})$.

42 Tillandsia kolbii W. Till \& Schatzl, Pl. Syst. Evol. 38: 259-262, f. 1. $1981 . \quad$ Fig. 17 Specimens examined: District Juxtlahuaca, Santiago Juxtlahuaca, S. Schatzl 79/55 (UAMIZ), S. Schatzl s. n. (WU).

43 Tillandsia lagunaensis Ehlers, Bromelie 2005(3): 80-82, figs. 2005. ENDEMIC. Fig. 16 Specimens examined: District Ixtlán, Capulalpam de Méndez, S. Figueroa B. \& F. Y. Guzmán R. 216 (UAMIZ(x8)); District Putla, Putla Villa de Guerrero, R. Ehlers 
EM051905 (MEXU(x2)). District tlaxiaco, Heroica Ciudad de Tlaxiaco, $R$. Ehlers \& K. Ehlers EM912602 (MEXU(x4)).

44 Tillandsia lampropoda L. B. Sm., in Yunck., Field Mus. Nat. Hist., Bot. Ser. 17: 320321, t. 9. 1938.

Specimens examined: District Juchitán, Santo Domingo Zanatepec, $R$. Torres $C$. \& $C$. Martínez 4839 (MEXU).

45 Tillandsia laui Matuda, Cact. Suc. Mex. 20: 96-97, f. 48. 1975.

Fig. 16

Specimens eXamined: District Juxtlahuaca, Santiago Juxtlahuca, A. Lau s. n. (MEXU?).

46 Tillandsia lautneri Ehlers, Bromelie 1993(2): 38-40, figs.1993.

Fig. 16 Specimens examined: District Mixe, Mixistlán de la Reforma, $R$. Torres $C$., L. Cortés A. \& P. Tenorio L. 8192 (MEXU); Totontepec Villa de Morelos, R. Torres C. \& C. Martínez 4989 (MEXU).

47 Tillandsia leiboldiana Schltdl., Linnaea 18: 414-415. 1844 [1845].

Fig. 17 Specimens examined: District Tehuantepec, Guevea de Humboldt, R. Torres C. \& C. Martínez 5949 (MEXU, MO). District Teotitlán, Eloxochitlán de Flores Magón, X. Munn \& F. Mendoza 1237 (MEXU), R. E. Schultes \& B. P. Reko 415 (GH, UC); San José Tenango, E. Torres B. 326 (UAMIZ).

48 Tillandsia leucolepis L. B. Sm., Phytologia 8: 497, t. 1, f. 1-3. 1963. Endemic. Fig. 17 Specimens eXamined: District Yautepec, Nejapa de Madero, T. Macdougall 280 (GH, NY, $\mathrm{US}(\mathrm{x} 4))$.

49 Tillandsia limbata Schltdl., Linnaea 18: 419-422. 1844 [1845].

Fig. 17 Specimens examined: District Tuxtepec, San José Chiltepec, A. R. López-Ferrari, A. Espejo, J. Ceja, A. Mendoza R., B. Pérez G. \& I. Reyes J. 2104 (UAMIZ(x4)); Santa María Jacatepec, A. R. López-Ferrari, A. Espejo \& G. Carnevali 3188 (UAMIZ), R. Torres C. \& E. Martínez S. 11037 bis (MEXU), R. Torres C. 12158 (MO).

50 Tillandsia loxichaensis Ehlers, Bromelie 2002(3): 78, f. 2002. EndEMIC. $\quad$ Fig. 17 Specimens examined: District Miahuatlán, Miahuatlán de Porfirío Diaz, K. Ehlers \& $R$. Ehlers EM891804 (MEXU).

51 Tillandsia lucida E. Morren ex Baker, Handb. Bromel. 207-208. 1889.

Fig. 17

Specimens examined: District Choapam, Santiago Choapam, B. P. Reko 4099 (US). DisTRICT IXTLÁN, Ixtlán de Juárez, J. Rzedowski 34034 (ENCB); Santiago Comaltepec, J. Rzedowski 30654 (ENCB). District Juchitán, San Miguel Chimalapa, T. MacDougall bromel 138 (GH, US(x2)). District Mixe, Totontepec Villa de Morelos, J. Rivera R. \& G. J. Martin 1256 (MEXU). District Teotitlán, Huautla de Jiménez, J. Utley \& K. Burt-Utley 6951 (MEXU). District Tlacolula, Santo Domingo Albarradas, J. Ceja, A. Espejo, A. 
Espejo-Serna et al.: Bromeliad flora of Oaxaca

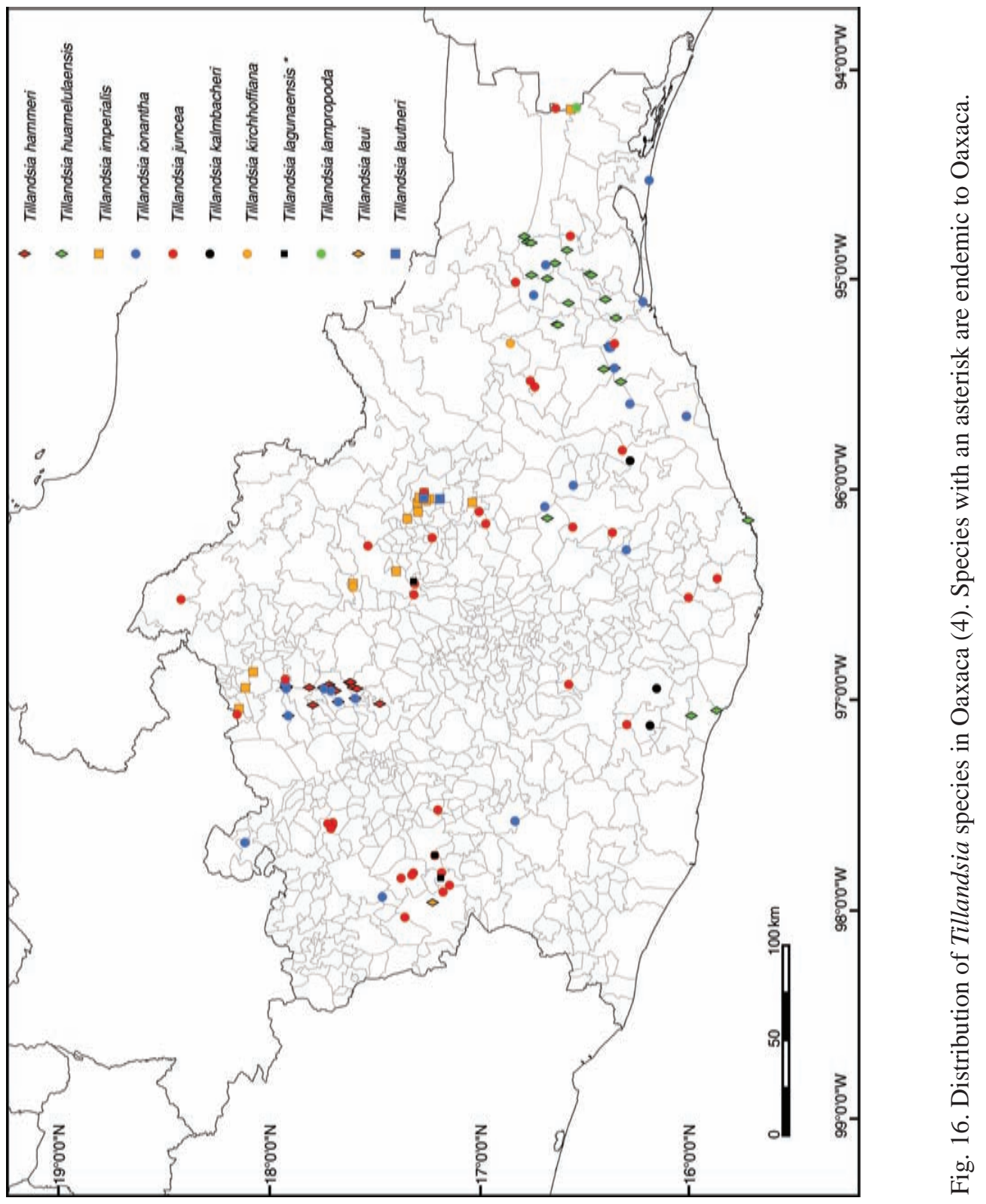


R. López-Ferrari \& A. Mendoza R. 1598 (UAMIZ(x2)). District Villa Alta, San Andrés Yaá, R. Torres C. \& E. Ramírez 8521 (MEXU).

52 Tillandsia macdougallii L. B. Sm., Contr. U. S. Natl. Herb. 29: 277-278, f. 2. 1949.

Fig. 17

Specimens examined: District Coixtlahuaca, D. H. Lorence \& A. García-Mendoza 4792 (MEXU); San Pedro Nopala, A. García-Mendoza, D. Frame, P. Tenorio L. \& A. Salinas T. 2327 (MEXU), A. García-Mendoza, R. Torres C. \& A. Campos V. 3952 (IEB); Santa María Nativitas, A. García-Mendoza, D. Frame, P. Tenorio L., A. Salinas T. \& C. Rincón 2362 (MEXU, MO), A. Salinas T., P. Tenorio L., D. Frame \& A. García-Mendoza F-3334-b (MEXU, MO); Tepelmeme Villa de Morelos, R. Cruz C. 2165 (ENCB), P. Tenorio L., C. Romero de T. \& E. Martínez C. 9330 (MEXU), P. Tenorio L., A. Salinas T., D. Frame \& A. García-Mendoza 11684 (MEXU, US), P. Tenorio L. \& L. Kelly 21320 (MEXU). District Etla, San Pablo Etla, C. Conzatti 76 (GH, MEXU), M. Urbina s. n. (MEXU); Santiago Tlazoyaltepec, T. MacDougall 343 (US). District Huajuapam, San Andrés Dinicuiti, W. Boege 3012 (ENCB, MEXU); Santa Catarina Zapoquila, P. Tenorio L. \& C. Romero de T. 11233 (MEXU). District Ixtlán, Capulalpam de Méndez, R. Cedillo T., D. H. Lorence \& A. García-Mendoza 954 (ENCB, MEXU, MO); Ixtlán de Juárez, R. Cedillo T. \& D. H. Lorence 2321 (LL, MEXU, MO), A. R. López-Ferrari, A. Espejo, J. Ceja, A. Mendoza R. \& G. Carnevali 3083 (UAMIZ), D. H. Lorence, M., G. Lorence \& R. Cedillo T. 3607 (MEXU, MO), G. McPherson 700 (ENCB, MICH); Santa Catarina Ixtepeji, A. Espejo, A. R. LópezFerrari, M. Flores C. \& J. Santana C. 4075 (MEXU, UAMIZ(x2), XAL), A. R. López-Ferrari, A. Espejo, J. Ceja, A. Mendoza R. \& G. Carnevali 3070 (UAMIZ), A. Saynes V. 1118 (ENCB, MEXU, XAL), J. Utley \& K. Burt-Utley 6671 (MEXU); Santa Catarina Lachatao, T. MacDougall s. n. (US); Santiago Xiacuí, W. Karwinski s. n. (OXF). District MiahUATLÁn, San Juan Mixtepec, E. Hunn OAX-91 (MEXU), E. Hunn OAX-309 (MEXU), E. Hunn 1891 (MEXU). District Nochixtlán, Asunción Nochixtlán, A. García-Mendoza \& F. Mérida 2603 (MEXU), O. van Hyning 5956 (US). District Teposcolula, San Juan Teposcolula, M. L. Torres C. 1065 (MEXU); San Pedro Nopala, P. Tenorio L. 20294 (MEXU); San Pedro y San Pablo Teposcolula, R. Torres C. \& M. L. Torres C. 12338 (MEXU); Villa Tejupam de la Unión, A. Espejo, A. R. López-Ferrari, J. Ceja \& A. Mendoza R. 6223 (UAMIZ), A. García-Mendoza, R. Torres C. \& L. Cortés A. 3086 (MEXU). District Tlacolula, San Lorenzo Albarradas, $R$. Torres C., D. H. Lorence \& A. García-Mendoza 3959 (ENCB, MEXU, MO). Without precise locality, A. Lau Jr. sub D. Cathcart s. n. (SEL), F. Kubisch s. n. (US), G. J. Martin GJM-M310 (MEXU).

53 Tillandsia magnusiana Wittm., Bot. Jahrb. Syst. 11: 66. 1889; L. B. Sm., Phytologia 19: 287. 1970. Fig. 17

Specimens examined: District Etla, San Jerónimo Sosola, F. A. Barkley \& D. J. Carr 36190 (GH). District Juquila, San Juan Lachao, J. Ceja, A. Espejo, A. R. López-Ferrari \& A. Mendoza R. 1285 (UAMIZ). District Pochutla, San Pedro el Alto, A. Espejo, A. R. López-Ferrari, J. Ceja \& A. Mendoza R. 6326 (IEB, UAMIZ). District Sola de Vega, Villa Sola de Vega, J. Ceja, A. Espejo, A. R. López-Ferrari \& A. Mendoza R. 1271 (CICY, UA- 
MIZ). District Tlacolula, San Pedro Totolapa, A. A. Reznicek, R. S. W. Bobbete et al. 66 (MICH). District Tlaxiaco, Santiago Yosondúa, J. Ceja, A. Espejo, A. R. López-Ferrari, A. Mendoza R. \& G. Carnevali 1756 (UAMIZ), J. Ceja, A. Espejo, A. R. López-Ferrari, A. Mendoza R. \& G. Carnevali 1762 (UAMIZ). District Villa Alta, San Francisco Cajonos, H. H. Iltis 27147 (MEXU).

54 Tillandsia makoyana Baker, Hand. Bromel. 188. 1889.

Fig. 17

Specimens examined: District Centro, Oaxaca de Juárez, A. R. López-Ferrari, A. Espejo, J. Santana C. \& M. Flores C. 1176 (UAMIZ). District Coixtlahuaca, Tepelmeme Villa de Morelos, A. Espejo, A. R. López-Ferrari, J. Ceja \& A. Mendoza R. 6311 (IEB(x2), UAMIZ(X3)), A. Salinas T. 7742 (MEXU). District Cuicatlán, Concepción Pápalo, A. Salinas T. \& R. Martínez S. 5937 (MEXU); San Pedro Jocotipac, A. Salinas T., G. Flores F. \& S. Bravo S. 4616 (MEXU, MO); Santiago Nacaltepec, C. A. Cruz Espinosa \& E. Martínez S. 335 (MEXU, UAMIZ(x2)). District EtLA, San Felipe Tejalapam, J. Santana C., R. Grether \& A. Martínez B. 323 (UAMIZ(x2)); San Francisco Telixtlahuca, A. Espejo, A. R. López-Ferrari, J. Ceja, A. Mendoza R. \& I. Ramírez M. 6493 (UAMIZ), A. García-Mendoza, D. H. Lorence \& D. Frame 1731 (MEXU); San Jerónimo Sosola, A. Salinas T. \& E. Martínez C. 6255 (MEXU), A. Salinas T., E. Martínez C. \& R. Martínez S. 6937 (MEXU); San Pablo Etla, A. R. López-Ferrari, A. Espejo, J. García-Cruz, E. Yáñez G. \& S. Acosta C. 1858 (UAMIZ(x4)). District Huajuapam, Mariscala de Juárez, J. I. Calzada \& A. Campos V. 18340 (MEXU); San Andrés Dinicuiti, W. Boege 3017 (MEXU); San Marcos Arteaga, R. Torres C. \& R. Cedillo T. 2636 (MEXU); San Pedro y San Pablo Tequixtepec, A. Paz Z. 215 (MEXU), A. Salinas T., O. Dorado R. \& J. L. Villaseñor R. F-3128 (MEXU). District IxtLÁn, Capulalpam de Méndez, S. Figueroa B. \& F. Y. Guzmán R. 448 (CHAP); Santa Catarina Ixtepeji, G. J. Martin GJM-M292 (MEXU, MO). District Juchitán, Asunción Ixtaltepec, J. Meave del C. \& E. Pérez G. 1767 (CICY); El Barrio de la Soledad, A. Espejo, A. R. López-Ferrari, J. Ceja, A. Mendoza R. \& I. Ramírez M. 6480 (UAMIZ). District JuxtlahuacA, San Juan Mixtepec, A. García-Mendoza, A. Campos V. \& J. Reyes 3637 (IEB, MEXU), J. Reyes S. 1520a (MEXU); Santiago Juxtlahuaca, A. Espejo, A. R. LópezFerrari, J. Ceja, A. Mendoza R. \& G. Carnevali 6867 (UAMIZ(x2); Santos Reyes Tepejillo, J. I. Calzada 19721 (MEXU(x2)). District Miahuatlán, San Jerónimo Coatlán, A. García-Mendoza, A. Campos V. \& J. Reyes 4666 (MEXU). District Putla, Putla Villa de Guerrero, A. Espejo, A. R. López-Ferrari, J. Ceja \& A. Mendoza R. 6792 (UAMIZ(x3)). District Tehuantepec, Magdalena Tequisistlán, C. Martínez R. 1399 (MEXU); Santiago Laollaga, $R$. Torres C., L. Cortés A. \& M. P. Ramírez 9090 (MEXU, MO); Santo Domingo Tehuantepec, C. Martínez R. 432 (FCME), M. L. Torres C., R. Torres C. \& C. Martínez 970 (MEXU). District Teotitlán, Santa María Ixcatlán, A. Salinas T. \& P. Tenorio L. 5854a (MEXU), A. Salinas T. \& E. Martínez C. 6117 (MEXU), A. Salinas T., E. Martínez C. \& R. Martínez S. 6463 (MEXU), P. Tenorio L. \& E. Martínez C. 17782 (MEXU); Teotitlán de Flores Magón, A. García-Mendoza, A. Salinas T. \& E. Martínez S. 3367 (IEB, MEXU), A. Salinas T. \& C. H. Ramos F-3922 (IEB, MEXU, MO), R. Torres C. \& M. A. Martínez 6637 (IEB, MEXU). District Teposcolula, San Juan Teposcolula, R. Torres C. \& J. L. Villaseñor 5082 (MEXU); Villa de Tamazulapam del Progreso, A. García-Mendoza, F. 
Martínez \& F. Mérida 2044 (MEXU), A. García-Mendoza, R. Torres C. \& L. Cortés A. 3105 (MEXU). District Tlacolula, San Jerónimo Tlacochahuaya, A. García-Mendoza, L. Torres \& L. Cortés A. 2834 (MEXU); San Lorenzo Albarradas, J. Ceja, A. Espejo, A. R. López-Ferrari \& A. Mendoza R. 1570 (UAMIZ(x3)); San Pablo Villa de Mitla, J. Ceja, A. Espejo, A. R. López-Ferrari \& A. Mendoza R. 1566 (UAMIZ(x4)), M. Cházaro B., M. Kimnach \& M. Negrete A. 6790 (MEXU, MICH); San Pedro Totolapa, C. S. Gardner 254 (US), E. W. Nelson 2554 (US), M. B. Foster \& O. Van Hyning 2929 (US(x2)). District TlaxiAco, San Mateo Peñasco, A. Espejo, A. R. López-Ferrari, J. Ceja \& A. Mendoza R. 6256 (UAMIZ(x3)); Santiago Yosondúa, J. Ceja, A. Espejo, A. R. López-Ferrari, A. Mendoza R. \& G. Carnevali 1760 (UAMIZ), J. Ceja, A. Espejo, A. R. López-Ferrari, A. Mendoza R. \& G. Carnevali 1764 (UAMIZ). District Yautepec, San Carlos Yautepec, J. Ceja, A. Espejo, A. R. López-Ferrari, A. Mendoza R. \& I. Ramírez M. 1384 (UAMIZ(x2)); Santa María Ecatepec, P. Tenorio L. 18868 (MEXU). Without precise locality, C. Conzatti \& V. González 1145 (GH, US).

55 Tillandsia makrinii L. Hrom., Bromelie 1994(1): 2-4, figs. 1994. ENDEMIC. Fig. 17 Specimens examined: District Pochutla, San Miguel del Puerto, J. Rivera H., S. Salas M. \& M. Elorsa C. $2218 a$ (MEXU); San Pedro Pochutla, H. Hromadnik \& L. Hromadnik 16100 (WU(x4)), E. Makrinius 722 (US).

56 Tillandsia x marceloi Takizawa \& P. Koide, J. Bromeliad Soc. 49: 213. 1999. EndEMic. Fig. 17

Specimens examined: District Ixtlán, Guelatao de Juárez, H. Takizawa, P. Koide, W. Schuster, D. Kinnard \& C. Lau s. n. (SEL)

57 Tillandsia maritima Matuda, Cact. Suc. Mex. 16: 90-91, f. 47. $1971 . \quad$ Fig. 17 Specimens examined: District Pochutla, San Miguel del Puerto, F. López 22 (MEXU), J. Rivera H., S. Salas M. \& M. Elorsa C. 2218 (UAMIZ), E. Martínez S., M. Elorsa C. \& C. Perret 32043 (MEXU); A. Saynes V., A. Sánchez \& S. Salas H. $3829 b$ (MEXU). District Tehuantepec, San Pedro Huamelula, S. Salas M., M. Elorsa C. \& L. Schibli 3624 (MEXU), R. Torres C. \& C. Martínez 4740 (MEXU).

58 Tillandsia mitlaensis W. Weber \& Ehlers, in W. Weber, Feddes Repert. 94: 617-618, f. 13. 1983. ENDEMIC. Fig. 18 Specimens examined: District Tlacolula, San Pablo Villa de Mitla, C. Delgadillo M. 175 (MEXU), K. Ehlers \& R. Ehlers s. n. (WU(x2)), W. R. Ernst 2286 (MEXU(x2), US), E. Matuda 38649 (MEXU), C. G. Pringle 13856 (MICH, US), C. G. Pringle 13857 (GH, US). District Centro, Santa María del Tule, K. Ehlers \& R. Ehlers EM 851601 (MEXU(x2)), J. Lautner LW 1981/20 (MEXU).

59 Tillandsia mixtecorum Ehlers \& Koide, J. Bromeliad Soc. 48: 52-54, f. 1-2. 1998. ENDEMIC.

Specimens examined: District Tlaxiaco, Santa María Yucuhiti, P. Koide \& A. Lau 9110294 (MEXU, SEL, WU(x2)). 
Espejo-Serna et al.: Bromeliad flora of Oaxaca

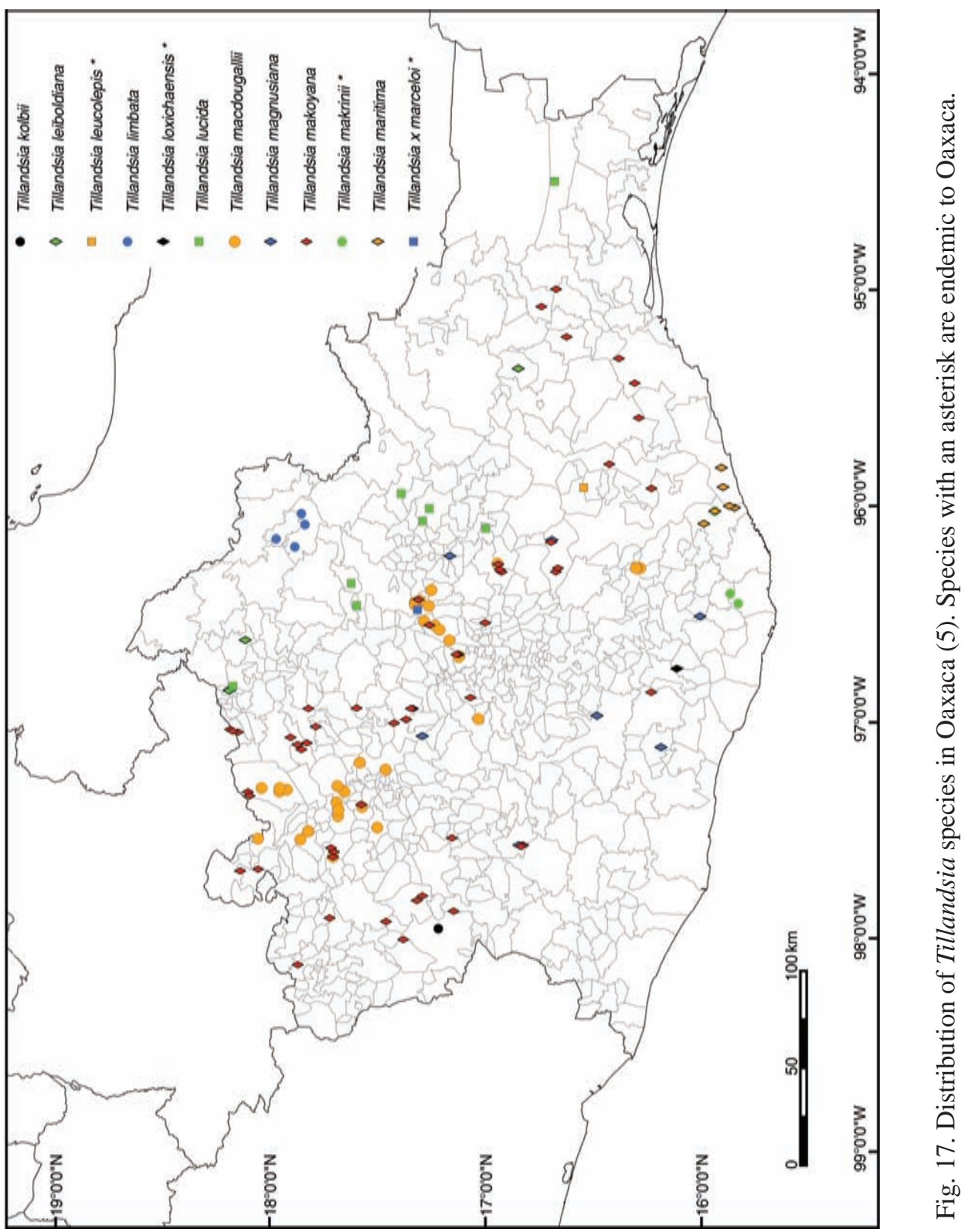


60 Tillandsia multicaulis Steud., Nomencl. bot. ed. 2. 2: 688. $1841 . \quad$ Fig. 18 Specimens examined: District IxtLÁn, Santa Catarina Lachatao, G. J. Martin GJM-M257 (MEXU, MO); Santiago Comaltepec, R. Cedillo T., R. Torres C. \& D. H. Lorence 1229 (IBUG, IEB, MEXU), R. López L. \& G. J. Martin 689 (MEXU), T. MacDougall 424 ([S. \& D., 1979] US). District Mixe, San Pedro y San Pablo Ayutla, G. J. Martin GJM-M211 (MEXU, MO); Totontepec Villa de Morelos, F. Liebmann 7955 ([S. \& D., 1979] C, GH, US), E. Ramírez G. \& P. Ramírez C. 5 (MEXU), E. Ramírez G. \& P. Ramírez C. 353 (MEXU), E. Ramírez G. \& P. Ramírez C. 354 (MEXU), J. Rivera R. \& G. J. Martin 1319 (MEXU). District Tehuantepec, Guevea de Humboldt, $R$. Torres C., P. Tenorio L. \& C. Romero de T. 2459 (ENCB, MEXU, MO). District Teotitlán, Huautla de Jiménez, T. B. Croat 48319 (MO), X. Munn \& F. Mendoza 1371 (MEXU); Mazatlán Villa de Flores, $R$. Torres C. \& R. Cedillo T. 1481 (MEXU); San José Tenango, S. Salas M. 353 (MEXU, UAMIZ). District Tlacolula, Santo Domingo Albarradas, J. Ceja, A. Espejo, A. R. López-Ferrari \& A. Mendoza R. 1596 (UAMIZ(x4)). Distric Tuxtepec, San Felipe Usila, C. Gallardo, J. Meave, G. Ibarra, A. Rincón, R. de Santiago, R. González \& P. Osorio 1095 (MEXU), S. Salas M., E. Torres B., J. Rivera H. \& R. García S. 1228 (SERO, UAMIZ). District Villa Alta, San Andrés Yaá, R. Torres C., M. L. Torres C. \& C. Martínez 7082 (MEXU); San Francisco Cajonos, H. H. Iltis 27145 (MEXU); San Juan Juquila Vijanos, S. Acosta C. 2468 (UAMIZ).

61 Tillandsia nolleriana Ehlers, in Rauh, Trop. Subtrop. Pflanzenw. 58: 29-32 (739-742), f. 18-20. 1986. ENDEMIC.

Specimens examined: District Juquila, San Juan Lachao, S. Acosta C. 2295 (UAMIZ), $R$. Ehlers 82/85 (HEID(x3), SEL), J. Rzedowski 19612 (MICH(x2), TEX, US(x2)). District Miahuatlán, San Jerónimo Coatlán, A. Campos V. 1565 (MEXU, MO), A. Campos V. 3506 (MEXU), A. García-Mendoza, A. Campos V. \& J. Reyes 4651 (MEXU). District Pochutla, San Pedro Pochutla, $D$. Cathcart s. $n$. (SEL). District Sola de Vega, Villa Sola de Vega, P. Tenorio L. \& J. L. Linares 19772 (MEXU). Without precise locality, S. Zamudio R. 9460 (IEB).

62 Tillandsia nuyooensis Ehlers, Bromelie 1989(3): 53-55, figs. 1989. ENDEMIC. Fig. 18 Specimens examined: District Ixtlán, Santa Catarina Ixtepeji, T. MacDougall s. n. (US). District Putla, Putla Villa de Guerrero, A. Espejo, A. R. López-Ferrari, J. Ceja \& A. Mendoza R. 6791 (UAMIZ(X3)). District Tlaxiaco, San Martín Itunyoso, R. Ehlers \& K. Ehlers EM882104 (WU(X8)). District YautePec, San Carlos Yautepec, T. MacDougall 272 sub E. Matuda 26072 (US).

63 Tillandsia oaxacana L. B. Sm., Contr. U. S. Natl. Herb. 29: 279-280, f. 4. 1949.

Specimens examined: District Centro, Tlalixtac de Cabrera, A. A. Reznicek, $R$. S. W. Bobbete et al. M226 (MICH). District Etla, San Juan del Estado, G. J. Martin GJM-M282 (MO). District Huajuapam, San Andrés Dinicuiti, W. Boege 3014 (MEXU); Santo Domingo Tonalá A. Lau s. n. (MEXU ?). District IxtLÁn, Capulalpam de Méndez, S. Figueroa B. \& F. Y. Guzmán R. 199 (UAMIZ); Santa Catarina Ixtepeji, H. H. Iltis 27138 (MEXU); 
Santa Catarina Lachatao, H. H. Iltis 27139 (MEXU), T. MacDougall s. $n$. (US). District Miahuatlán, Miahuatlán de Porfirio Díaz, P. Koide 3 (SEL). District Putla, Putla Villa de Guerrero, A. Espejo, A. R. López-Ferrari, J. Ceja \& A. Mendoza R. 6790 (UAMIZ). District ZaAchila, San Pablo Cuatro Venados, A. G. Miranda M. 820 (CHAP). Without precise locality, E. Matuda 38663 (MEXU).

64 Tillandsia ortgiesiana E. Morren ex Mez, in C. DC., Monogr. Phan. 9: 678. 1896.

Fig. 18

Specimens examined: District Juchitán, Asunción Ixtaltepec, C. Gallardo H. \& E. Pérez G. 1475 (MEXU(x2)); Ciudad Ixtepec, T. MacDougall s. n. (US).

65 Tillandsia paucifolia Baker, Gard. Chron. n.s. 10: 748. 1878.

Fig. 18 Specimens examined: District Juchitán, Ciudad Ixtepec, $R$. Merrill King 1470 (TEX, US); Santiago Niltepec, $R$. Merrill King 1797 (US). District Tehuantepec, Santo Domingo Tehuantepec M. B. Foster \& O. Van Hyning 2940 (US).

66 Tillandsia polita L. B. Sm. var. elongata Ehlers, Bromelie 2003(2): 45, f. 2003. Fig. 18 Specimens examined: District Centro, Oaxaca de Juárez, F. Kubisch 18 (US). District IxtLÁn, Guelatao de Juárez O. van Hyning 6050 (US); San Juan Chicomezúchil, A. R. López-Ferrari, A. Espejo, J. Ceja, A. Mendoza R. \& G. Carnevali 3080 bis (UAMIZ(x2)). District Tlacolula, Santo Domingo Albarradas, J. Ceja, A. Espejo, A. R. López-Ferrari \& A. Mendoza R. 1592 (UAMIZ(x4)).

67 Tillandsia polystachia (L.) L., Sp. Pl. ed. 2. 410. 1762.

Fig. 18 Specimens examined: District Choapam, Santiago Yaveo, A. García-Mendoza, $R$. Torres C. \& L. Cortés A. 2916 (IEB, MEXU). District IxtLÁn, Santiago Comaltepec, $R$. E. Gereau \& G. J. Martin 1979 (MEXU, MO). District Juchitán, Santa María Chimalapa, S. Salas M. 1390 (MEXU). District Tuxtepec, San Juan Bautista Tuxtepec, T. P. Ramamoorthy, M. Sousa S., H. Hernández \& L. Cortés A. 1641 (MEXU); San Juan Bautista Valle Nacional, D. Thurm, D. Dunn, S. Trott, P. Case \& C. Dziekanowski 221 (ENCB, MEXU); Santa María Jacatepec, A. R. López-Ferrari, A. Espejo \& G. Carnevali 3192 (UAMIZ), C. H. Ramos \& E. Martínez S. 345 (CHAP), R. Torres C. \& E. Martínez S. 11037 (MEXU, MO), R. Torres C. \& L. Cortés A. 11491 (MEXU), R. Torres C. \& L. Cortés A. 11512 (MEXU), R. Torres C. 12155 (MEXU), J. Utley \& K. Burt-Utley 6742 (MEXU). District Villa Alta, San Francisco Cajonos, $R$. Torres C., P. Tenorio L. \& C. Romero de T. 2895 (MEXU); Villa Hidalgo, R. E. Gereau \& A. J. Moyer 2172 (MO).

68 Tillandsia prodigiosa (Lem.) Baker, J. Bot. 26: 140. 1888.

Fig. 18 Specimens examined: District Ejutla, La Compañía, W. Rauh RM 16018 B (HEID). DisTrict IxtLán, Capulalpam de Méndez, T. B. Croat \& D. P. Hannon 65656 (MEXU(X2), MO), S. Figueroa B. \& F. Y. Guzmán R. 198 (CHAP); Santa Catarina Ixtepeji, R. Cedillo T. \& D. H. Lorence 637 (MEXU, MO), S. D. Koch \& M. González L. 86113 (ENCB), A. R. López-Ferrari, A. Espejo, J. Ceja, A. Mendoza R. \& G. Carnevali 3069 (UAMIZ(x6)), F. Miranda 8389 (MEXU(x2)), D. H. Norris \& D. J. Taranto 16661 (MEXU); Santa Catarina 


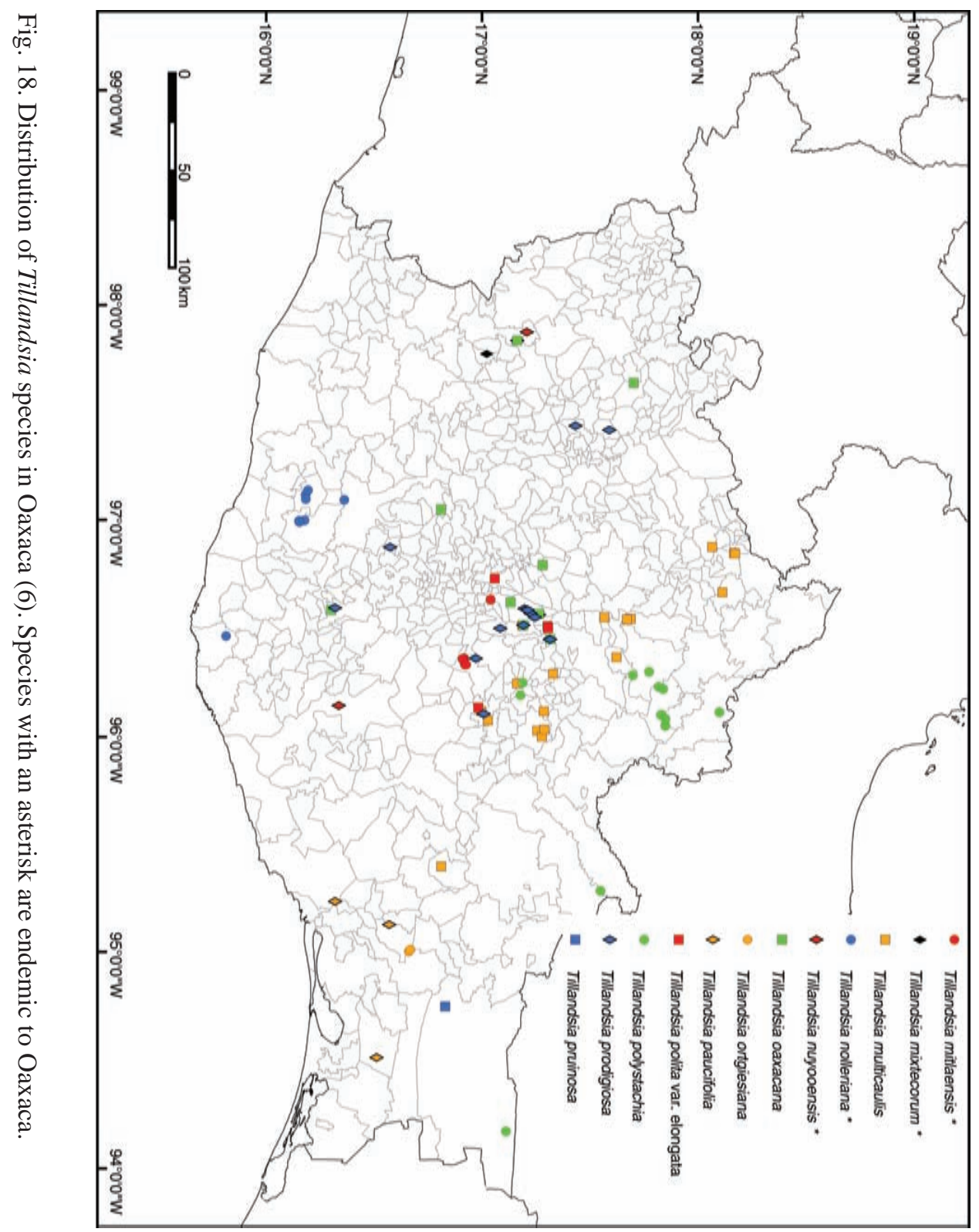


Lachatao, H. H. Iltis 27148 (MEXU). District Miahuatlán, Miahuatlán de Porfirio Díaz, S. C. Solano \& M. A. Vara 243 (MEXU). District Teposcolula, San Juan Teposcolula, A. García-Mendoza \& J. Reyes 4962 (MEXU); San Vicente Nuñu, A. García-Mendoza \& J. Reyes 5211 (MEXU). District Tlacolula, San Pablo Villa de Mitla, W. R. Ernst 2466 (MEXU(x3), MICH, US(x2)); Santo Domingo Albarradas, A. Espejo, A. R. López-Ferrari, J. Ceja \& A. Mendoza R. 6676 (UAMIZ(x4)); Teotitlán del Valle, M. Cházaro B., M. Kimnach, R. Dorsch \& M. Negrete 6831 (MEXU, XAL). Without precise locality, P. Koide s. $n$. (F); T. MacDougall s. n. (MO).

69 Tillandsia pruinosa Sw., Fl. Ind. Occid. 1: 594. 1797.

Fig. 18 Specimens eXamined: District Juchitán, Santa María Chimalapa, R. Torres C. \& E. Martínez S. 11117 (MEXU).

70 Tillandsia pseudobaileyi C. S. Gardner, Selbyana 7: 363, f. 2, 4, 5.1984.

Fig. 20

Specimens examined: District Tehuantepec, San Pedro Huamelula, M. Elorsa C. 1496 (SERO).

71 Tillandsia pseudosetacea Ehlers \& Rauh, Trop. Subtrop. Pflanzenw. 58: 35-37 (745747), f. 21. 1986.

Fig. 19

Specimens examined: District Juquila, San Juan Lachao, R. Ehlers EM82015 (HEID, WU(x3)), A. R. López-Ferrari, A. Espejo, J. Ceja \& A. Mendoza R. 2929 (IEB, UAMIZ). District Pochutla, San Pedro Pochutla, C. Conzatti, B. P. Reko \& Makrinius 3028 (MEXU), B. P. Reko 3028 (US).

72 Tillandsia pueblensis L. B. Sm., Contr. Gray Herb. 104: 81-82, t. 3, f. 1-2. 1934.

Fig. 19

Specimens examined: District Coixtlahuaca, Tepelmeme Villa de Morelos, A. GarcíaMendoza \& L. de la Rosa 6490 (MEXU). District Cuicatlán, San Juan Bautista Cuicatlán, C. A. Cruz Espinosa \& E. San Pedro 525 (UAMIZ(x2)). Without precise locality, A. Lau Jr. sub D. Cathcart s. n. (SEL).

73 Tillandsia punctulata Schltdl. \& Cham., Linnaea 6: 53-54. 1831.

Fig. 19 Specimens examined: District Ixtlán, Santiago Comaltepec, G. Davidse, M. Sousa, O. Téllez, E. Martínez \& J. Davidse 30225 (MEXU, MO), G. J. Martin 651 (UC), R. Merrill King 2114 (MICH), C. Sánchez A. 125 (ENCB), J. Utley \& K. Burt-Utley 6734 (MEXU). District Juchitán, San Miguel Chimalapa, M. Ishiki I. 1453 (MEXU(x2)), without colector 1104 (UAMIZ); Santa María Chimalapa, J. Rivera H., S. Salas M., R. García S. \& E. Torres B. 306 (UAMIZ); Santo Domingo Zanatepec, R. Torres C. \& C. Martínez 4848 (MEXU). District Mixe, San Pedro y San Pablo Ayutla, M. Cházaro B., M. Kimnach \& M. Negrete A. 6803 (MEXU, MICH, TEX, XAL), R. Torres C., M. L. Torres C. \& C. Martínez 7041 (MEXU); Totontepec Villa de Morelos, E. Ramírez G. \& P. Ramírez C. 380 (MEXU), J. Rivera R. \& G. J. Martin 773 (MEXU), E. Velasco L. \& G. J. Martin 109 (MEXU). District Tlacolula, Santo Domingo Albarradas, J. Ceja, A. Espejo, A. R. López-Ferrari \& A. Mendoza R. 1590 (UAMIZ), A. Espejo, A. R. López-Ferrari, J. Ceja \& A. Mendoza R. 6681 
(UAMIZ(X3)). District Tuxtepec, San Felipe Usila, C. Gallardo, J. Meave, G. Ibarra M., \& A. Rincón G. 822 (MEXU), C. Gallardo H., A. Rincón G., N. Velázquez \& M. Ocampo 933 (MEXU), A. Rincón G., C. Gallardo H., S. Espinoza \& N. Flores 374 (MEXU). District Villa Alta, San Andrés Yaá, R. Torres C., M. L. Torres C. \& C. Martínez 7082 bis (MEXU, MO); San Francisco Cajonos, H. H. Iltis 27146 (MEXU); San Melchor Betaza, $R$. Torres C. \& R. Cedillo T. 2030 (MEXU, MO).

74 Tillandsia quaquaflorifera Matuda, Cact. Suc. Mex. 22: 23-24, f. 14. $1977 . \quad$ Fig. 19 Specimens examined: District Ixtlán, Santa Catarina Ixtepeji, G. J. Martin GJM-M294 (MEXU).

75 Tillandsia recurvata (L.) L., Sp. Pl. ed. 2. 410. 1762.

Fig. 19 Specimens examined: District Centro, Oaxaca de Juárez, A. R. López-Ferrari \& A. Espejo 994 (UAMIZ), A. R. López-Ferrari, A. Espejo, J. Santana C. \& M. Flores C. 1172 (IEB, MEXU, UAMIZ), A. R. López-Ferrari, A. Espejo, J. Santana C. \& M. Flores C. 1182 BIS (UAMIZ); Santa Cruz Xoxocotlán, U. Reynosa G. 51 (MEXU); Tlalixtac de Cabrera, J. Conrad \& R. Conrad 3066 (MEXU). District Coixtlahuaca, San Miguel Tequixtepec, A. Salinas T. \& P. Solís S. F-3519 (MEXU). District Cuicatlán, San Juan Bautista Cuicatlán, C. A. Cruz Espinosa \& E. San Pedro 497 (MEXU, UAMIZ(x2)); San Pedro Jocotipac, A. Salinas T., G. Flores F. \& S. Bravo S. 4630 (MEXU). District EtLA, San Francisco Telixtlahuca, A. Espejo, A. R. López-Ferrari, J. Ceja, A. Mendoza R. \& I. Ramírez M. 6491 (UAMIZ), I. Ramírez M., A. Espejo, A. R. López Ferrari, A. Mendoza R. \& J. Ceja 1013 (UAMIZ); San Pablo Huitzo, F. Palma C. s. n. (MEXU). District Huajuapam, San Andrés Dinicuiti, A. García-Mendoza, F. Martínez \& F. Mérida 2054 (MEXU), O. Téllez, E. Martínez S. \& L. Rico 6222 (MEXU); San Pedro y San Pablo Tequixtepec, R. Torres C. \& J. L. Villaseñor 5003 (MEXU); Santiago Chazumba, B. Leuenberger \& C. Schiers 2564 (MEXU), A. G. Miranda M. 71 (CHAP), seminario de P. Dávila 24 (UAMIZ); Santiago Huajolotitlán, B. Bartholomew, L. R. Landrum, H. W. Li, T. S. Ying \& O. R. Dorado R. 3030 (MEXU), R. Torres C., R. Cedillo T. \& L. Cortés A. 770 (MEXU), R. Torres C. \& P. Tenorio L. 12798 (MEXU). District Ixtlán, Capulalpam de Méndez, S. Figueroa B. \& F. Y. Guzmán R. 373 (CHAP). District Juchitán, Asunción Ixtaltepec, J. Meave del C. \& E. Pérez G. 1848 (CICY, MEXU). District Miahuatlán, San Juan Mixtepec, E. Hunn OAX-862 (MEXU); San Sebastián Río Hondo, J. Calónico S. 26744 (UAMIZ). District Ocotlán, Santo Tomás Jalieza, A. Saynes V. 1156 (UAMIZ). District Silacayoapam, Ixpantepec Nieves, A. Espejo, A. R. López-Ferrari, J. Ceja, A. Mendoza R. \& G. Carnevali 6868 (UAMIZ). District Tehuantepec, Santo Domingo Tehuantepec, C. Martínez R. 69 (IEB, MEXU). District Teotitlán, San Jerónimo Tecoatl, U. Reynosa G. 54 (MEXU); Teotitlán de Flores Magón, A. Salinas T. \& C. H. Ramos F-3933 (IEB). District Teposcolula, Villa de Tamazulapam del Progreso, A. García-Mendoza, R. Torres C. \& L. Cortés A. 3106 (MEXU). District Tlacolula, San Lorenzo Albarradas, J. Santana C., A. R. López-Ferrari, M. Flores C. \& A. Espejo 31 (UAMIZ); San Pablo Villa de Mitla, J. Ceja, A. Espejo, A. R. López-Ferrari \& A. Mendoza R. 1567 (UAMIZ); San Pedro Totolapa, A. Saynes V. 1198 (FCME, IEB); Tlacolula de Matamoros, A. Espejo \& A. R. López-Ferrari 6764 (UAMIZ); Villa Díaz Ordaz, R. Torres C., D. Neil \& F. Neil 2162 (MEXU). District 
Tlaxiaco, Heroica Ciudad de Tlaxiaco, A. Espejo, A. R. López-Ferrari, J. Ceja \& A. Mendoza R. 6777 (UAMIZ). District Villa Alta, Villa Hidalgo, R. E. Gereau \& A. J. Moyer 2170 (MEXU). Without precise locality, E. Matuda 28444-A (MEXU).

76 Tillandsia rettigiana Mez, Repert. Spec. Nov. Regni Veg. 14: 249. $1916 . \quad$ Fig. 19 Specimens examined: District Pochutla, San Pedro el Alto, A. Espejo, A. R. López-Ferrari, J. Ceja \& A. Mendoza R. 6321 (UAMIZ(x5)).

77 Tillandsia rhodocephala Ehlers \& Koide, J. Bromeliad Soc. 44: 131-133, f. 16-17. 1994. ENDEMIC.

Specimens examined: District Tlaxiaco, Santiago Yosondúa, P. Koide \& A. Lau 9103191 (WU), P. Koide \& A. Lau sub H. Luther s. n. (SEL).

78 Tillandsia rubrispica Ehlers \& Koide, J. Bromeliad Soc. 46: 7-10, f. 5-8. 1996. ENDEMIC.

Fig. 19 Specimens examined: District Tlaxiaco, Santiago Yosondúa, J. Ceja, A. Espejo, A. R. López-Ferrari, A. Mendoza R. \& G. Carnevali 1753 (UAMIZ(x6)), R. Ehlers \& K. Ehlers 931203 (MEXU, WU(x7)), P. Koide \& A. Lau s. n. (WU(x3)).

79 Tillandsia schatzlii Rauh, Trop. Subtrop. Pflanzenw. 27: 8-10 (426-428), f. 3. 1979.

Specimens examined: District Etla, San Juan Bautista Atatlahuca, A. Espejo, A. R. LópezFerrari, J. Ceja, A. Mendoza R. \& I. Ramírez M. 6513 (UAMIZ), J. Rutschmann s. n. (US).

80 Tillandsia schiedeana Steud., Nomecl. bot. ed. 2. 2: 688. 1841.

Fig. 19 Specimens examined: District Cuicatlán, Concepción Pápalo, A. Salinas T. \& R. Martínez S. 5936 (MEXU); San Juan Bautista Cuicatlán, C. A. Cruz Espinosa, E. Martínez S. \& E. San Pedro 454 (MEXU, UAMIZ(x2)), C. A. Cruz Espinosa \& E. San Pedro 502 (MEXU, UAMIZ(x2)), E. Martínez S., J. L. Rebolledo C. \& V. Torres H. 33376 (MEXU), E. Martínez S. \& V. Torres H. 33543 (MEXU); San Pedro Teutila, K. Velasco G. \& G. Juárez 75 (UAMIZ); Santiago Nacaltepec, C. A. Cruz Espinosa \& E. Martínez S. 334 (MEXU, UAMIZ(x2)); Santos Reyes Pápalo, R. Torres C. \& J. L. Villaseñor 5060 (MEXU). DisTrict Сhoаpam, San Juan Lalana, A. García-Mendoza, R. Torres C. \& L. Cortés A. 2970 (IEB, MEXU); Santiago Choapam, R. Torres C., D. H. Lorence \& A. García-Mendoza 2623 (MEXU), R. Torres C., D. H. Lorence \& A. García-Mendoza 4027 (XAL); Santiago Yaveo, A. García-Mendoza, R. Torres C. \& L. Cortés A. 2915 (MEXU). District Ejutla, La Compañía, J. Ceja, A. Espejo, A. R. López-Ferrari \& A. Mendoza R. 1264 (UAMIZ). District ETLA, Magdalena Apasco, B. Leuenberger \& C. Schiers 2742 (MEXU); San Jerónimo Sosola, A. Salinas T. \& E. Martínez C. 6278 (MEXU); San Juan Bautista Jayacatlán, L. C. Smith S 550 (GH, MEXU). District Huajuapam, San Marcos Arteaga, R. Torres C. \& R. Cedillo T. 2623 (MEXU). District Ixtlán, Ixtlán de Juárez, R. García S. 551 (MEXU); San Juan Chicomezúchil, A. R. López-Ferrari, A. Espejo, J. Ceja, A. Mendoza R. \& G. Carnevali 3079 (UAMIZ). District Juchitán, Asunción Ixtaltepec, A. Espejo, A. R. López-Ferrari, J. Ceja, A. Mendoza R. \& I. Ramírez M. 6484 (UAMIZ), C. Martínez R. 1270 
(MEXU), J. Meave del C., E. Pérez G., C. Gallardo, B. Reyes \& J. Reyes 1777 (MEXU), J. Meave del C. \& E. Pérez G. 1847 (MEXU(x2)); San Miguel Chimalapa, T. MacDougall s. n. ([S. \& D., 1979] US), S. Maya J. 1058 (MEXU), S. Maya J. 1210 (MEXU, UAMIZ); San Pedro Tapanatepec, A. Reyes 1336 (CHAP); Santa María Chimalapa, H. Hernández G. 787 (UAMIZ), H. Hernández G. 912 (UAMIZ), T. Wendt, S. \& C. Hernández G. 5905 (UAMIZ). District Juxtlahuaca, San Juan Mixtepec, J. Reyes S. 1352 (MEXU); Santos Reyes Tepejillo, J. I. Calzada 20898 (MEXU). District Miahuatlán, Miahuatlán de Porfirio Díaz, R. Torres C., D. Neil \& F. Neil 2205 (MEXU). District Mixe, Totontepec Villa de Morelos, J. Rivera R. \& G. J. Martin 543 (MEXU), J. Rivera R. \& G. J. Martin 1799 (MEXU(x2)), J. Rivera R. JR2464 (MEXU). District Putla, Putla Villa de Guerrero, D. H. Lorence, A. García-Mendoza \& R. Cedillo T. 3416 (MEXU). District Tehuantepec, San Pedro Huamelula, S. Salas M. 2070 (MEXU, UAMIZ); Santo Domingo Tehuantepec, C. Martínez R. 1316 (MEXU). District Teotitlán, San Bartolomé Ayautla, $R$. Torres C., $R$. Cedillo T. \& L. Cortés A. 826 (MEXU); San Juan de los Cues, A. Salinas T. \& C. H. Ramos F-3975 (IEB, MEXU, XAL); Santa María Ixcatlán, P. Tenorio L. \& E. Martínez C. 17396 (MEXU). District Tlacolula, San Dionisio Ocotepec, $R$. Torres C. \& P. Tenorio L. 8211 (IEB, MEXU); San Pedro Totolapa, M. Cházaro B., M. Kimnach, R. Dorsch \& M. Negrete 6864 (XAL), R. Ehlers s. n. sub W. Weber 472 ([Feddes Repert 94: 595 HAL]), B. Hansen, J. Hansen \& M. Nee 1577 (MEXU, MICH), T. Morley 677 (MEXU), E. W. Nelson 2544 ([S. \& D., 1979] US); Santa María Zoquitlán, M. Cerón C., S. Salas M. \& H. Morales 237 (SERO, UAMIZ); Santo Domingo Albarradas, J. Ceja, A. Espejo, A. R. López-Ferrari \& A. Mendoza R. 1587 (UAMIZ). District Tlaxiaco, San Agustín Tlacotepec, J. Ceja, A. Espejo, A. R. López-Ferrari, A. Mendoza R. \& G. Carnevali 1748 (UAMIZ); Santiago Nuyoó, A. García-Mendoza, A. de Ávila, L. Cervantes \& A. Saynes 6831 (MEXU). District Tuxtepec, Acatlán de Pérez Figueroa, E. Guízar N. \& J. C. Echevarría 5718 (CHAP); Nuevo Soyaltepec, L. Cortés A., R. Torres C., A. García-Mendoza \& G. Ibarra M. 742 (MEXU), L. Cortés A., R. Torres C., A. García-Mendoza \& G. Ibarra M. 753 (CHAP), L. Cortés A., R. Torres C., M. Sousa S. \& G. Andrade M. 868 (MEXU), R. E. Gereau, M. Sousa S., R. Torres C. \& L. Cortés A. 2242 (MEXU); San José Chiltepec, G. Martínez C. 553 ([S. \& D., 1979] GH); San Juan Bautista Tuxtepec, A. R. López-Ferrari, A. Espejo \& G. Carnevali 3187 (UAMIZ); San Juan Bautista Valle Nacional, R. Cedillo T. \& R. Torres C. 1051 (MEXU, XAL), T. B. Croat 39731 (MEXU); San Pedro Ixcatlán, L. Cortés A., R. Torres C. \& P. Tenorio L. 180 (MEXU); Santa María Jacatepec, C. H. Ramos \& E. Martínez S. 288 (CHAP), C. H. Ramos \& E. Martínez S. 312 (CHAP, XAL), C. H. Ramos \& E. Martínez S. 611 (CHAP), E. Martínez S. \& C. H. Ramos 24022 (IEB, MEXU), R. Torres C. \& E. Martínez S. 11016 (MEXU(x2)). District Villa Alta, Villa Hidalgo, R. E. Gereau \& A. J. Moyer 2174 (MEXU). District Yautepec, Nejapa de Madero, J. Ceja, A. Espejo, A. R. López-Ferrari, A. Mendoza R. \& I. Ramírez M. 1389 (UAMIZ); San Carlos Yautepec, T. B. Croat 46251 (MEXU); San Juan Lajarcia, R. Torres C., L. Cortés A. \& C. Martínez 9861 (MEXU).

81 Tillandsia schusteri Rauh, Trop. Subtrop. Pflanzenw. 58: 37-41 (747-751), f. 22-22a. 1986. ENDEMIC.

Fig. 19

Specimens examined: District Cuicatlán, Santiago Nacaltepec, W. Schuster s. $n$. $(\operatorname{HEID}(\mathrm{x} 2))$. 
Espejo-Serna et al.: Bromeliad flora of Oaxaca

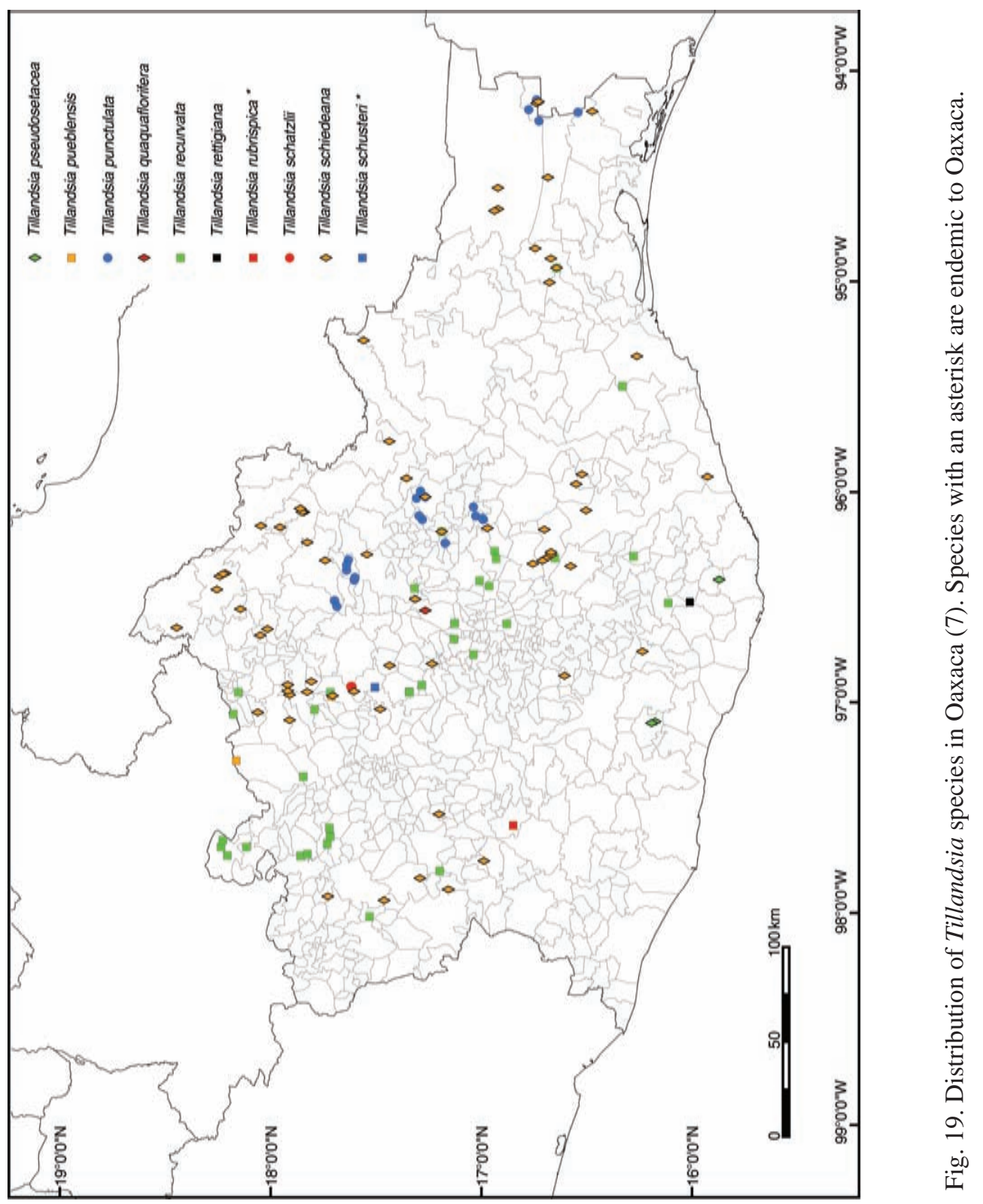


82 Tillandsia seleriana Mez, Bot. Jahrb. Syst. 30 (Beibl. 67): 8. 1901.

Fig. 20 Specimens examined: District Juchitán, Asunción Ixtaltepec, P. Tenorio L., R. Torres C. \& C. Romero de T. 3443 (ENCB, MEXU, MO), R. Torres C. \& E. Martínez S. 11210 (MEXU, TEX); El Barrio de la Soledad, A. Espejo, A. R. López-Ferrari, J. Ceja, A. Mendoza R. \& I. Ramírez M. 6476 (UAMIZ); San Miguel Chimalapa, C. Perret, J. Rivera H., C. Perret, H. Asborgsen, S. Solórzano, J. Avendaño, Felipe \& Elsa 111 (MEXU); Santo Domingo Zanatepec, $R$. Torres C. \& C. Martínez 4846 (MEXU, MO). District Sola de Vega, San Ildefonso Sola, V. García G. 14 (UAMIZ(x2)).

83 Tillandsia setiformis Ehlers, Bromelie 1993(1): 3-6, figs. 1993. ENDEMIC.

Fig. 20 Specimens examined: District Putla, Putla Villa de Guerrero, M. Cházaro B., M. Kimnach \& M. Negrete A. 7087 (MEXU, MICH), K. Ehlers \& R. Ehlers EM821103 ([Bromelie 1993(1): WU]), K. Ehlers \& R. Ehlers EM912604 (SEL, WU(x2)), A. Espejo, A. R. LópezFerrari, J. Ceja \& A. Mendoza R. 6788 (UAMIZ(x2)). District Tlaxiaco, Heroica Ciudad de Tlaxiaco, E. Matuda 38290 (MEXU, US); Santiago Nuyoó, R. Ehlers \& K. Ehlers EM882002 (WU(x2)), R. Ehlers M882003 (WU(x2)); Santo Tomás Ocotepec, K. Ehlers \& R. Ehlers EM8210105 ([Bromelie 1993(1): WU]).

84 Tillandsia sierrajuarezensis Matuda, Cact. Suc. Mex. 18: 50-51, f. 24. 1973. ENDEMic.

Fig. 20

Specimens examined: District Centro, Tlalixtac de Cabrera, G. L. Webster, B. Hallberg, K. Miller \& L. Miller 11484 (MEXU, US). District Etla, San Pablo Etla, C. Conzatti 700 (GH), V. González \& C. Conzatti 710 (GH). District Ixtlán, San Juan Evangelista Analco, O. van Hyning 5972 ([S. \& D., 1979] US); San Pablo Macuiltianguis, C. Delgadillo M. 108 (MEXU), J. Utley \& K. Burt-Utley 6995 (MEXU); Santa Catarina Ixtepeji, R. E. Gereau \& G. J. Martin 1935 (ENCB, MEXU(x2), MO(x2)), G. J. Martin GJM-M017 (MEXU), E. Matuda 38420 (CODAGEM(x2), MEXU(x3)), R. Torres C. \& A. García-Mendoza 8051 (MEXU), J. Utley \& K. Burt-Utley 6678 (MEXU); Santiago Comaltepec, E. Matuda \& colaboradores 38376 (MEXU); Santiago Xiacuí, A. R. López-Ferrari, A. Espejo, J. Ceja, A. Mendoza R. \& G. Carnevali 3090 (UAMIZ(x3)). District Mixe, Mixistlán de la Reforma, A. Espejo, A. R. López-Ferrari, J. Ceja \& A. Mendoza R. 6674 (UAMIZ(x6)). District Villa Alta, Villa Hidalgo, W. R. Ernst 2346 (MEXU(x2)). District Yautepec, San Carlos Yautepec, E. Matuda \& colaboradores 38375 (MEXU), E. Matuda \& colaboradores 38377 (MEXU), E. Matuda \& colaboradores 38379 (MEXU). Without precise locality, C. Conzatti 1149 (GH, MEXU).

85 Tillandsia socialis L. B. Sm., Phytologia 6: 257, t. 1, f. 1-2. 1958.

Fig. 20

Specimens examined: District Juchitán, El Barrio de la Soledad, S. Zamudio 6357 (IEB, UAMIZ).

86 Tillandsia streptophylla Scheidw. ex E. Morren, Hort. Belge 3: 252. t. $1836 . \quad$ Fig. 20 Specimens examined: District Choapam, Santiago Yaveo, R. E. Schultes 562 (ECON). DisTrict Juchitán, Asunción Ixtaltepec, A. Espejo, A. R. López-Ferrari, J. Ceja, A. Mendoza R. \& I. Ramírez M. 6482 (UAMIZ), R. Torres C. \& J. L. Villaseñor 5169 (MEXU, MO), R. 
Torres C. \& E. Martínez S. 11209 (MEXU); San Juan Guichicovi, R. Torres C., L. Cortés A. \& A. Campos V. 12090 (MEXU), W. G. Williams Jr. \& D. Francoeur 42A (MICH). District Tuxtepec, Loma Bonita, J. Chavelas P., C. Zamora \& G. Soria R. ES-4190 (MEXU).

87 Tillandsia supermexicana Matuda, Cact. Suc. Mex. 22: 21-22, f. 12. $1977 . \quad$ Fig. 20 Specimens examined: District Juquila, San Juan Lachao, H. Hromadnik \& L. Hromadnik 16092 (WU(x4)). District Miahuatlán, San Jerónimo Coatlán, R. Ehlers \& K. Ehlers EM912109 ([Bromelie 1993(2): WU]), H. Hromadnik \& L. Hromadnik 16113 ([Bromelie 1993(2): WU]). District Putla, Putla Villa de Guerrero, H. Hromadnik \& L. Hromadnik 14164 (WU(x3)). District Sola de Vega, San Ildefonso Sola, V. García G. 10 (UAmiz(x3)). District Tlaxiaco, Heroica Ciudad de Tlaxiaco, A. Espejo, A. R. LópezFerrari, J. Ceja \& A. Mendoza R. 6776 (UAMIZ(x4)).

88 Tillandsia tonalaensis Ehlers, J. Bromeliad Soc. 53: 17-19, figs. 6-8. 2003. ENDEMIC.

Fig. 20

Specimens examined: District Huajuapam, Santo Domingo Tonalá, R. Ehlers 882111 (US, WU(x2)), K. Ehlers \& R. Ehlers EM 892305 ([J. Bromeliad Soc. 53(1): 16 MEXU]), $R$. Ehlers \& K. Ehlers 930602 (WU). District Juxtlahuaca, San Sebastián Tecomaxtlahuaca, K. Ehlers \& R. Ehlers EM 930701 ([J. Bromeliad Soc. 53(1): 16 MEXU]).

89 Tillandsia tricolor Schltdl. \& Cham., Linnaea 6: 54. 1831.

Fig. 20

Specimens examined: District Juchitán, Santa María Chimalapa, M. A. Pérez Farrera 2222 (UNICACH). District Mixe, Totontepec Villa de Morelos, J. Rivera R. \& G. J. Martin 1306 (MEXU). District Tehuantepec, Guevea de Humboldt, A. Campos V. \& R. Torres C. 3563 (MEXU), R. Torres C., P. Tenorio L. \& C. Romero de T. 2451 (ENCB, MEXU, MO), R. Torres C., L. Cortés A. \& M. P. Ramírez 9134 (MEXU); Santa María Guienagati, P. Tenorio L., R. Torres C. \& C. Martínez 11176 (IEB, MEXU); Santiago Lachiguiri, $R$. Torres C. \& C. Martínez 5729 (MEXU), R. Torres C. \& A. Campos V. 13906 (MEXU). DisTrict Teotitlán, Eloxochitlán de Flores Magón, X. Munn \& H. Munn 1212 (MEXU), R. E. Schultes \& B. P. Reko 271 (GH). Without precise locality, E. Matuda \& colaboradores 38539 (LL, MEXU).

90 Tillandisa ulrici Ehlers, Bromlie 2000: 66-68. fog. p. 65-66. 2000. EnDEMIC. Fig. 20 Specimens examined: District Tlaxiaco, Santiago Yosondúa, R. Ehlers, U. Lautner, J. Lautner \& B. Janke EM980404 (MEXU(x2), WU(x6)).

91 Tillandsia usneoides (L.) L., Sp. Pl. ed. 2. 411. 1762.

Fig. 20

Specimens examined: District Coixtlahuaca, San Cristóbal Suchixtlahuaca, D. Ramírez C. s. n. (MEXU). District Cuicatlán, San Pedro Jocotipac, A. Salinas T., G. Flores F. \& S. Bravo S. 4628 (FCME, MEXU). District EtLA, San Francisco Telixtlahuca, A. Espejo, A. R. López-Ferrari, J. Ceja, A. Mendoza R. \& I. Ramírez M. 6495 (UAMIZ); San Jerónimo Sosola, D. Benavides V. s. n. (MEXU), E. Guízar N. \& A. G. Miranda M. 4839 (MEXU); San Juan del Estado, G. J. Martin GJM-M273 (MEXU). District IxtLÁn, Capulalpam de Méndez, J. Ceja, A. Espejo, A. R. López-Ferrari, A. Mendoza R. \& G. Carnevali 1622 


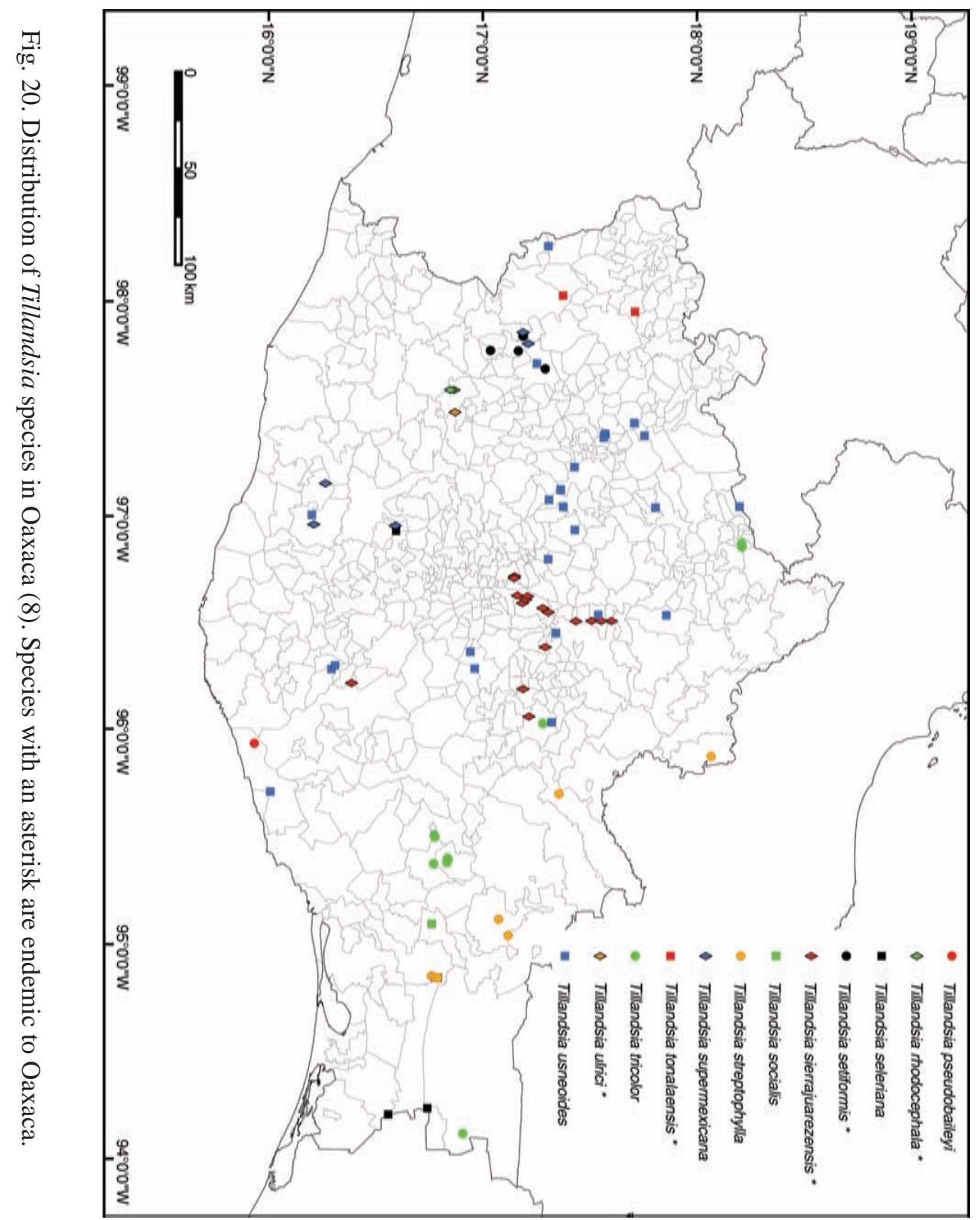


(UAMIZ); San Pablo Macuiltianguis, L. Hernández G. 216 (UAMIZ). District Juxtlahuaca, Coicoyán de las Flores, J. I. Calzada 19549 (IEB, MEXU). District Miahuatlán, San Jerónimo Coatlán, A. Campos V. \& R. Torres C. 1607 (MEXU); San Juan Mixtepec, E. Hunn 612 (MEXU), E. Hunn OAX-613 (MEXU); San Pedro Mixtepec, E. Hunn 1698 (MEXU). District Mixe, Totontepec Villa de Morelos, E. Velasco L. \& G. J. Martin 297 (MEXU(x2)). District Nochixtlán, Asunción Nochixtlán, A. García-Mendoza, D. H. Lorence \& R. Cedillo T. 366 (FCME, MEXU), D. H. Lorence, A. García-Mendoza \& R. Cedillo T. 3447 (IEB, MEXU); Magdalena Zahuatlán, B. Leuenberger \& C. Schiers s. n. (MEXU); Santo Domingo Yanhuitlán, G. Diggs et al. 3828 (MEXU), A. Espejo, A. R. López-Ferrari, J. Ceja, A. Mendoza R. \& G. Carnevali 6847 (UAMIZ), H. H. Iltis 1149 ([S. \& D., 1979] US, WIS). District Tehuantepec, San Pedro Huamelula, S. Acosta C. 949 (IEB). District Teotitlán, Teotitlán de Flores Magón, M. T. Germán, O. Téllez V. \& M. Sousa S. s. n. (FCME). District Teposcolula, Villa Tejupam de la Unión, J. I. Calzada 23299 (MEXU). District Tlacolula, San Lorenzo Albarradas, J. Ceja, A. Espejo, A. R. López-Ferrari \& A. Mendoza R. 1573 (UAMIZ); San Pablo Villa de Mitla, B. Hallberg 1052 ([S. \& D., 1979] MICH, US). District Tlaxiaco, Heroica Ciudad de Tlaxiaco, A. Espejo, A. R. López-Ferrari, J. Ceja \& A. Mendoza R. 6771 (UAMIZ). District Tuxtepec, San Felipe Usila, J. I. Calzada \& A. Graciano 16908 (B) (FCME, MEXU). Without precise locality, G. J. Martin GJM-M247 (MEXU).

92 Tillandsia utriculata L., Sp. Pl. 286. 1753.

Fig. 21 Specimens examined: District Tehuantepec, Guevea de Humboldt, A. Campos $V$. \& $R$. Torres C. 3562 (MEXU), R. Torres C. \& L. Cortés A. 8894 (MEXU); Santiago Lachiguiri, A. Campos V. \& R. Torres C. 3668 (MEXU). District Tuxtepec, Santa María Jacatepec, C. H. Ramos \& E. Martínez S. 305 (XAL).

93 Tillandsia variabilis Schltdl., Linnaea 18: 418. 1844 [1845].

Fig. 21

Specimens examined: District Choapam, San Juan Lalana, A. García-Mendoza, R. Torres C. \& L. Cortés A. 2969 (MEXU), A. García-Mendoza, L. Torres \& L. Cortés A. 2986 (MEXU). District Juchitán, Santa María Chimalapa, H. Hernández G. 987 (UAMIZ). District Putla, Putla Villa de Guerrero, T. B. Croat 45906 (MO), A. Espejo, A. R. LópezFerrari, J. Ceja \& A. Mendoza R. 6795 (UAMIZ). District Tuxtepec, Nuevo Soyaltepec, L. Cortés A., R. Torres C., A. García-Mendoza \& G. Ibarra M. 775 (MEXU); Santa María Jacatepec, A. R. López-Ferrari, A. Espejo \& G. Carnevali 3191 (UAMIZ(x2)), R. Torres C. \& E. Martínez S. 10986 (MEXU).

94 Tillandsia vernardoi Rauh, Trop. Subtrop. Pflanzenw. 16: 36-38 (238-240), f. 19. 1976. ENDEMIC.

Fig. 21 Specimens examined: District Tlacolula, San Pablo Villa de Mitla, J. Roth \& V. Magnuson s. $n$. (HEID).

95 Tillandsia violacea Baker, J. Bot. 25: 279. 1887.

Fig. 21 Specimens examined: District Coixtlahuaca, Tepelmeme Villa de Morelos, A. Salinas T., P. Tenorio L. \& J. Sánchez-Ken 5645 (MEXU). District Huajuapam, San Pedro y San 
Pablo Tequixtepec, A. G. Miranda, E. Guízar N. \& A. Z. Alvarado 1094 (CHAP, UAMIZ). District Teotitlán, San Francisco Huehuetlán, C. Conzatti 4114 (MEXU); Santa María Chilchotla, E. Matuda 38725 (ENCB). District Teposcolula, Villa Tejupam de la Unión, A. García-Mendoza, R. Torres C. \& L. Cortés A. 3095 (MEXU). District Tlaxiaco, San Miguel el Grande, S. D. Koch \& M. González L. 86107 (ENCB).

96 Tillandsia viridiflora (Beer) Baker, J. Bot. 26: 81. 1888.

Fig. 21

Specimens examined: District Ixtlán, Ixtlán de Juárez J. Rzedowski 33348 (ENCB), J. Rzedowski 33826 (ENCB(x2)), J. Santana C. \& L. Pacheco 907A (UAMIZ(x3)); San Pedro Yólox, A. Espejo, A. R. López - Ferrari, J. Ceja, A. Mendoza R. \& G. Carnevali 6700 (UAMIZ(x12)); Santiago Comaltepec, S. D. Koch \& M. González L. 86123 (ENCB), J. Utley \& K. Burt-Utley 6278 (MEXU(x2)), O. van Hyning 5959 (US(x2). District Juchitán, San Miguel Chimalapa, T. MacDougall s. $n$. (US(x2)). District Tehuantepec, Santiago Lachiguiri, A. Campos V. \& R. Torres C. 3679 (MEXU). District Teotitlán, San Lucas Zoquiapam, R. E. Schultes \& B. P. Reko 410 (GH). District Villa Alta, San Juan Juquila Vijanos, X. Munn, R. Aguilar, R. del Castillo, R. Ramírez \& R. Rivera 275 (MEXU(x2)).

97 Tillandsia wuelfinghoffii Ehlers, J. Bromeliad Soc. 40: 201-202, f. 3-5. 1990. ENDEMIC. Fig. 21

Specimens examined: District IxtLÁn, Guelatao de Juárez, R. Ehlers \& K. Ehlers EM881601 (SEL, WU(x2)), R. Wulfinghoff 86-209(WU).

98 Tillandsia xerographica Rohweder, Senckenbergiana 34: 113, f. 8-11, t. 1, f. 2. 1953.

Fig. 21

Specimens examined: District Putla, Santa María Ipalapa, A. Espejo, A. R. López-Ferrari, J. Ceja \& A. Mendoza R. 6799 (UAMIZ(x7)). District Yautepec, San Carlos Yautepec, P. de Luca, S. Sabato \& Balduzzi s. n. (NY(x3)), P. de Luca \& S. Sabato s. n. (US); Santa María Ecatepec, T. MacDougall 402 (UC, US(x2)). Without precise locality, M. B. Foster 3029 ([S. \& D., 1979] US).

99 Tillandsia yerba-santae Ehlers, J. Bromeliad Soc. 40: 268-269, f. 10-12. 1990. EndEMIC.

Fig. 21

Specimens examined: District Tlaxiaco, Santiago Yosondúa, A. Lau s. n. (WU(x2)).

100 Tillandsia yunckeri L. B. Sm., in Yunck., Field. Mus. Nat. Hist., Bot. Ser. 17: 322, t. 10. 1938.

Fig. 21

Specimens examined: District Juchitán, San Miguel Chimalapa, T. MacDougall s. $n$. (GH), N. Velázquez, R. Soriano, R. López, S. Solano \& Gustavo 654 (MEXU).

101 Tillandsia sp. 1. ENDEMIC.

Fig. 21

Specimens examined: District Pochutla, San Pedro Pochutla, A. Espejo, A. R. López-Ferrari, J. Ceja \& A. Mendoza R. 6312 (UAMIZ(x3)); San Pedro el Alto, J. Rzedowski 20648 (ENCB(x3), MICH, TEX(x2)); San Pedro Pochutla, C. S. Gardner 1445 ((SEL, US). 
Espejo-Serna et al.: Bromeliad flora of Oaxaca

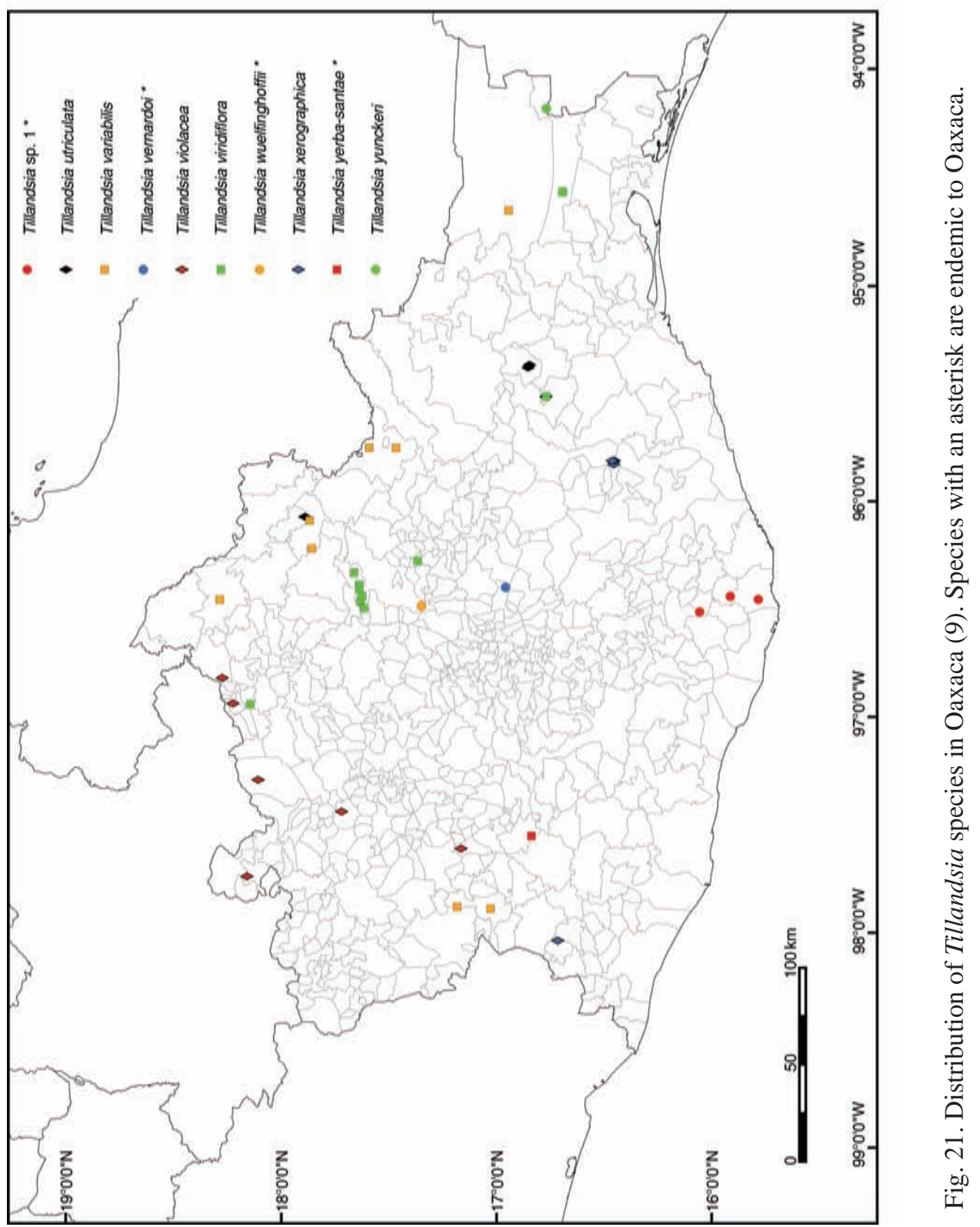


13 VIRIDANTHA

1 Viridantha atroviridipetala (Matuda) Espejo, Acta Bot. Mex. 60: 28-29, fig. 1, 2. 2002.

Fig. 22

Specimens examined: District Centro, Oaxaca de Juárez, A. R. López-Ferrari, A. Espejo, J. Santana C. \& M. Flores C. 1182 (UAMIZ). District Coixtlahuaca, Santa Magdalena Jicotlán, R. Cruz C. s. n. (ENCB); Tepelmeme Villa de Morelos, A. Espejo, A. R. López-Ferrari, J. Ceja \& A. Mendoza R. 6310 (IEB, UAMIZ), A. Salinas T. 7793 (MEXU). District Cuicatlán, San Pedro Jocotipac, A. Salinas T. \& E. Petterssen 4718 (MEXU). District Etla, San Francisco Telixtlahuca, A. Espejo, A. R. López-Ferrari, J. Ceja, A. Mendoza R. \& I. Ramírez M. 6492 (UAMIZ); San Jerónimo Sosola, A. Espejo, A. R. López-Ferrari \& J. Ceja 5318 (UAMIZ). District Huajuapam, San Andrés Dinicuiti, A. García-Mendoza, F. Martínez \& F. Mérida 2053 (MEXU); O. Téllez, E. Martínez S. \& L. Rico 6220 (MEXU), R. Torres C. \& C. Martínez 5002 (MEXU); San Juan Bautista Suchitepec, A. Salinas T. \& A. Campos V. F-3675 (MEXU, TEX); San Pedro y San Pablo Tequixtepec, A. Salinas T., J. L. Villaseñor \& O. Dorado R. F-3121 (MEXU, US); Santiago Chazumba, A. G. Miranda M. 1098 (CHAP); Tezoatlán de Segura y Luna, M. Cházaro B., M. Kimnach \& M. Negrete A. 7113 (IBUG, MICH, XAL). District IxtLÁn, Capulalpam de Méndez, S. Figueroa B. \& F. Y. Guzmán R. 403 (CHAP); Santa Catarina Ixtepeji, J. Utley \& K. Burt-Utley 6683 (MEXU). District Juxtlahuaca, Santos Reyes Tepejillo, J. I. Calzada 20057 (MEXU). District Miahuatlán, San Juan Mixtepec, E. Hunn 1925 (MEXU). District TeposcoluLA, Villa de Tamazulapam del Progreso, W. R. Anderson \& C. Anderson 5382 (MICH), J. I. Calzada 23949 (MEXU), B. Leuenberger \& C. Schiers 2779 (MEXU), J. Rzedowski 19698 (ENCB, MICH), O. van Hyning 5954 (US). District Tlacolula, San Jerónimo Tlacochahuaya, D. Barry, Jr. 50 (US); Santo Domingo Albarradas, J. Ceja, A. Espejo, A. R. López-Ferrari \& A. Mendoza R. 1584 (UAMIZ). District Tlaxiaco, San Mateo Peñasco, $A$. Espejo, A. R. López-Ferrari, J. Ceja \& A. Mendoza R. 6259 (UAMIZ); San Pedro Molinos, A. Espejo, A. R. López-Ferrari, J. Ceja \& A. Mendoza R. 6260 (IEB, UAMIZ).

2 Viridantha mauryana (L. B. Sm.) Espejo, Acta Bot. Mex. 60: 29, fig. 3. 2002. Fig. 22 Specimens examined: District Tlacolula, San Jerónimo Tlacochahuaya, J. Rzedowski 36520 (ENCB, IEB, MEXU); Tlacolula de Matamoros, C. Conzatti 3932 (US).

3 Viridantha penascoensis (Ehlers \& Lautner) Espejo \& López-Ferrari, comb. nov. ENDEMIC.

Fig. 22

Basyonim: Tillandsia penascoensis Ehlers \& Lautner, Bromelie 2004(1): 4-7, figs. 2004.

Specimens examined: District Tlaxiaco, San Mateo Peñasco, U. Lautner \& M. Kretz EM030202 (WU(x2), [Bromelie, 2004(1): MEXU]).

4 Viridantha plumosa (Baker) Espejo, Acta Bot. Mex. 60: 3, fig. 5. 2002.

Fig. 22

Specimens examined: District Centro, Santo Domingo Tomaltepec, G. J. Martin GJMM271 (MEXU, MO). District Coixtlahuaca, Tepelmeme Villa de Morelos, P. Tenorio L. 19960 (MEXU), P. Tenorio L. \& L. Kelly 20117 (MEXU); Tlacotepec Plumas, R. Cruz C. 
2311 (ENCB). District Cuicatlán, Santiago Nacaltepec, L. C. Smith 535 (GH). District Etla, San Francisco Telixtlahuca, A. Espejo, A. R. López-Ferrari, J. Ceja, A. Mendoza R. \& I. Ramírez M. 6494 (UAMIZ); San Jerónimo Sosola, F. A. Barkley \& D. J. Carr 36190 [bis] (GH). District Huajuapam, San Andrés Dinicuiti, W. Boege 3011 (MEXU). DisTrict IxtLÁn, San Miguel Amatlán, J. Ceja, A. Espejo, A. R. López-Ferrari, A. Mendoza R. \& G. Carnevali 1614 (UAMIZ); Santa Catarina Ixtepeji, A. R. López-Ferrari, A. Espejo, J. Ceja, A. Mendoza R. \& G. Carnevali 3072 (UAMIZ). District Juxtlahuaca, San Juan Mixtepec, J. Reyes S. 1424 (MEXU). District Miahuatlán, San Juan Mixtepec, E. Hunn OAX-533 (MEXU). District Nochixtlán, Asunción Nochixtlán, R. Cedillo T., D. H. Lorence \& A. García-Mendoza 835 (MEXU, MO), A. Espejo, A. R. López-Ferrari \& J. García-Cruz 5093 (UAMIZ); Magdalena Zahuatlán, B. Leuenberger \& C. Schiers 2775 (MEXU); San Pedro Cántaros Coxcaltepec, A. Salinas T. \& R. Martínez S. 5958 (MEXU); Santo Domingo Yanhuitlán, G. Diggs, R. Diggs, C. Greenfield \& D. Severinson 3829 (MEXU), A. Espejo, A. R. López-Ferrari, J. Ceja, A. Mendoza R. \& G. Carnevali 6845 (UAMIZ), H. H. Iltis, R. Koeppen \& F. Iltis 1144 (ENCB, MICH). District Teposcolula, San Juan Teposcolula, A. García-Mendoza 231 (MEXU); San Pedro Nopala, A. GarcíaMendoza, R. Torres C. \& L. Cortés A. 3077 (MEXU), A. García-Mendoza, R. Torres C. \& A. Campos V. 3953 (MEXU), A. Salinas T., D. Frame, P. Tenorio L. \& A. García-Mendoza F3317 (MEXU, MO, TEX), R. Torres C., A. García-Mendoza \& L. Cortés A. 9563 (MEXU); San Pedro Yucunama, D. H. Lorence, A. García-Mendoza \& R. Cedillo T. 3373 (ENCB, MEXU, MO). District Tlaxiaco, Chalcatongo de Hidalgo, A. Espejo, A. R. López-Ferrari \& J. Ceja 5335 (CHIP, UAMIZ, XAL); Heroica Ciudad de Tlaxiaco, A. Espejo, A. $R$. López-Ferrari, J. Ceja \& A. Mendoza R. 6778 (UAMIZ), A. Espejo, A. R. López-Ferrari, J. Ceja, A. Mendoza R. \& G. Carnevali 6860 (UAMIZ), R. McVaugh 22307 (ENCB, MEXU, MICH); San Martín Huamelulpam, A. Espejo, A. R. López-Ferrari, J. Ceja \& A. Mendoza R. 6237 (IEB, UAMIZ); San Pedro Molinos, A. Espejo, A. R. López-Ferrari, J. Ceja \& A. Mendoza R. 6261 (UAMIZ). Without precise locality H. Bravo H. 5 (MEXU(x2)), A. Lau Jr. sub D. Cathcart s. n. (SEL), C. Seler \& E. Seler 1606 (GH).

\section{VRIESEA}

1 Vriesea heliconioides (Kunth) Hook. ex Walp., Ann. Bot. (London) 3: 623. 1852. Fig. 22 Specimens examined: District Juchitán, Santa María Chimalapa, J. Rivera H., R. García S. \& J. C. Flores V. 1165 (MEXU), R. de Santiago G., J. Rivera H., T. Alvarez R. \& D. Acuca V. 652 (UAMIZ). District Tuxtepec, San Pedro Ixcatlán, J. I. Calzada 10268 (XAL).

2 Vriesea malzinei E. Morren, Belgique Hort. 24: 313, t. 14. 1874.

Specimens examined: District Juchitán, Matías Romero, C. Martínez R. 1096 (MEXU). District Tuxtepec, Acatlán de Pérez Figueroa, F. Salazar s. n. (MEXU); Nuevo Soyaltepec, L. Cortés A., R. Torres C., M. Sousa S. \& G. Andrade M. 865 (MEXU(x2)); San Felipe Usila, R. E. Schultes \& B. P. Reko 666 (GH(x2)). 


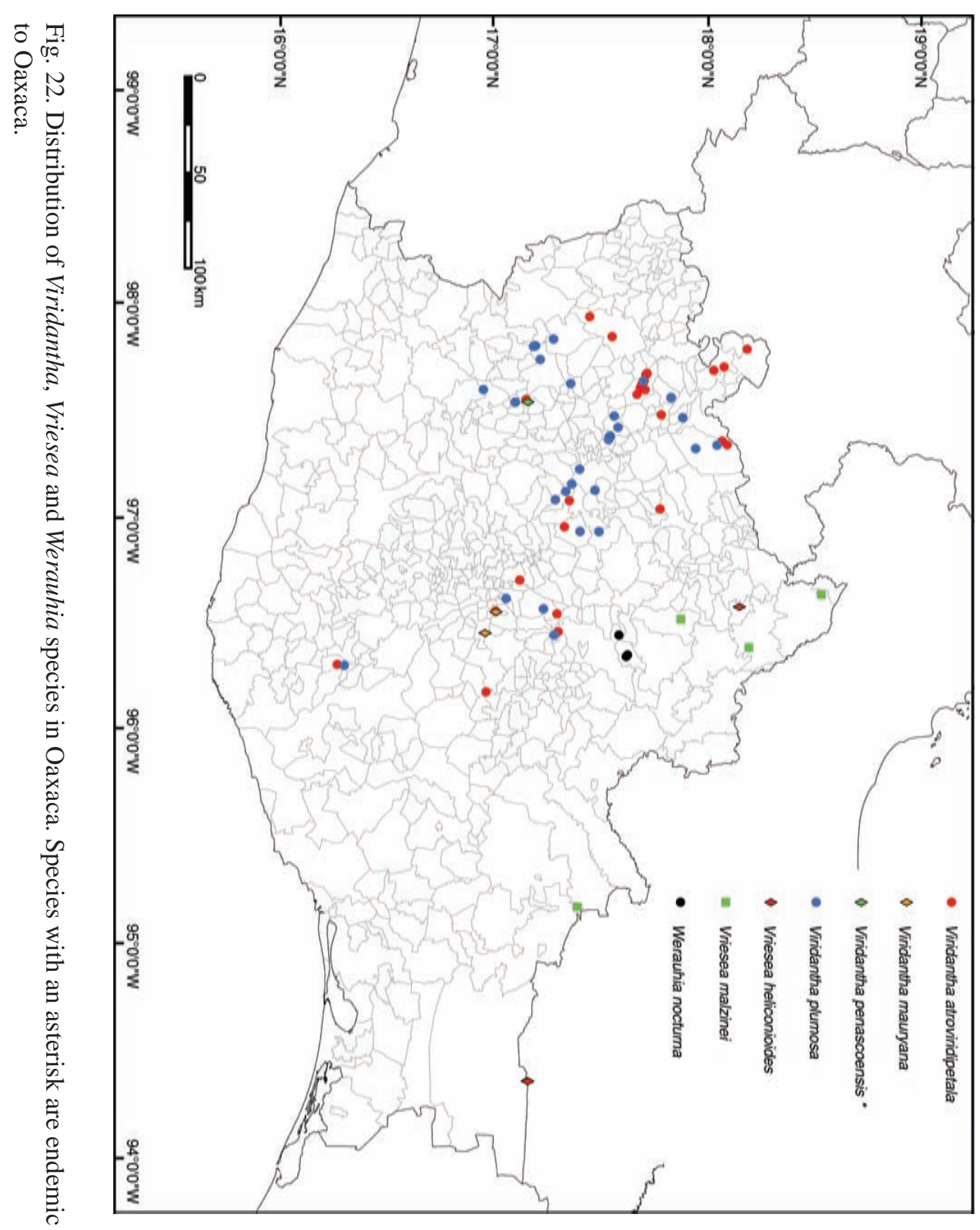


15 WERAUHIA

1 Werauhia gladioliflora (H. Wendl.) J. R. Grant, Trop. Subtrop. Pflanzenw. 91: 31-32. 1995.

Fig. 23

Specimens eXamined: District Juchitán, Santa María Chimalapa, J. Rivera H., R. García S. \& J. C. Flores V. 1167 (MEXU).

2 Werauhia nocturna (Matuda) J. R. Grant, Trop. Subtrop. Pflanzenw. 91: 33. 1995.

Fig. 22

Specimens examined: District Ixtlán, Santiago Comaltepec, C. R. Beutelspacher B. 73 (MEXU), J. Utley \& K. Burt-Utley 6731 (MEXU), O. van Hyning 6051 (US).

3 Werauhia nutans (L. B. Sm.) J. R. Grant, Phytologia 79: 255. 1995 [10 Jun 1996].

Fig. 23

Specimens examined: District Juchitán, Santa María Chimalapa, P. Tenorio L. \& T. L. Wendt 19335 (MEXU, UAMIZ).

4 Werauhia pycnantha (L. B. Sm.) J. R. Grant, Phytologia 78: 122.1995.

Fig. 23

Specimens examined: District Juchitán, San Miguel Chimalapa, T. MacDougall bromel 136 (US(x2)), T. Wendt, S. Maya \& M. Ishiki 5036 (UAMIZ).

5 Werauhia vanhyningii (L. B. Sm.) J. R. Grant, Trop. Subtrop. Pflanzenw. 91: 35. 1995.

Fig. 23

Specimens examined: District Ixtlán, Ixtlán de Juárez, J. Rzedowski 33931 (ENCB), J. Rzedowski 34035 (ENCB, MEXU, MICH), R. Torres C., A. García-Mendoza \& L. Cortés A. 9552 (IBUG, IEB, MEXU(x2)); San Juan Quiotepec, R. García S. 53 (SERO); Santiago Comaltepec, C. R. Beutelspacher B. 69 (MEXU), A. Espejo \& A. R. López-Ferrari 6682 (UAMIZ), A. García-Mendoza, R. Torres C. \& L. Cortés A. 3038 (MEXU), R. Merrill King 2127 (MICH), J. Rzedowski 30613 (ENCB, IEB), R. Torres C., L. Cortés A. \& C. Martínez 7827 (MEXU), R. Torres C. \& L. Cortés A. 10221 (MEXU), R. Torres C. \& E. Martínez S. 11326 (MEXU), R. Torres C. \& E. Martínez S. 11329 (MEXU), J. Utley \& K. Burt-Utley 6710 (MEXU), J. Utley \& K. Burt-Utley 7024 (MEXU(x2)), O. van Hyning 5961 (US), O. van Hyning s. $n$. (US(x2)). District Mixe, Totontepec Villa de Morelos, R. Torres C. \& O. Téllez 8647 (MEXU). District Tehuantepec, Guevea de Humboldt, $R$. Torres C. \& L. Cortés A. 14347 (MEXU). District Tuxtepec, San Felipe Usila, C. Gallardo H., A. Rincón G., N. Velázquez \& M. Ocampo 923bis (MEXU), C. Gallardo H., A. Rincón G., N. Velázquez \& M. Ocampo 979 (MEXU), C. Gallardo H., A. Rincón G., N. Velázquez \& M. Ocampo 994 (MEXU), C. Gallardo H. 1069 (MEXU), A. Rincón G., J. Meave del C., C. Gallardo H., A. Otero A. \& L. Lozada P. 233 (MEXU), E. Torres B., S. Salas M., J Rivera H. \& R. García S. 896 (SERO, UAMIZ). District Villa Alta, San Juan Juquila Vijanos, D. H. Lorence \& R. Cedillo T. 4081 (MEXU). 


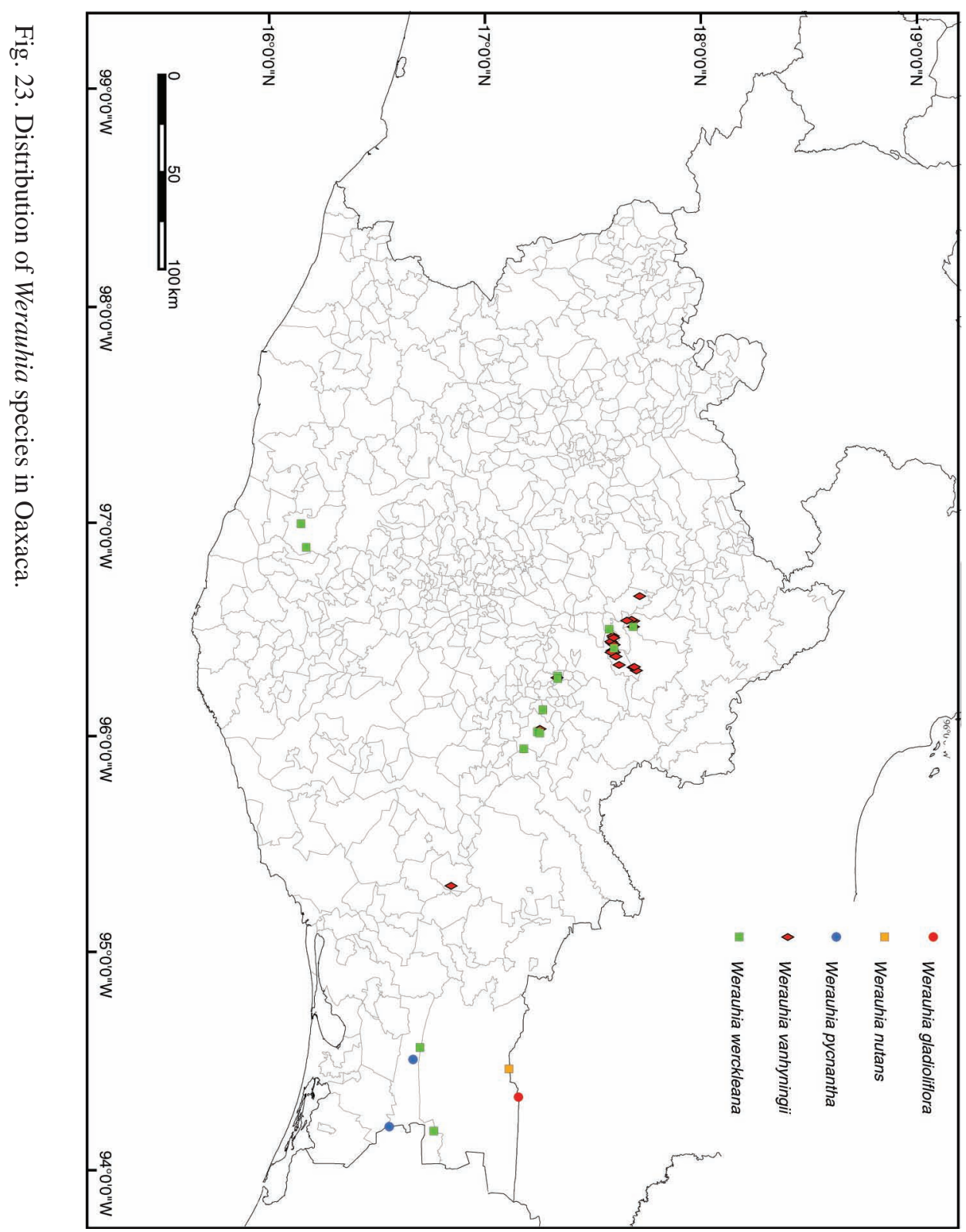


6 Werauhia werckleana (Mez) J. R. Grant, Trop. Subtrop. Pflanzenw. 91: 38-39. 1995.

Fig. 23

Specimens examined: District Ixtlán, San Pedro Yólox, A. Espejo, A. R. López - Ferrari, J. Ceja, A. Mendoza R. \& G. Carnevali 6698 (UAMIZ(x7)); Santiago Comaltepec, J. Utley \& K. Burt-Utley 6726 (MEXU). District Juchitán, San Miguel Chimalapa, M. Ishiki I. 1270 (MEXU(x2), MO), T. MacDougall s. n. (GH, US); without municipio indicated, T. MacDougall 296.S (US). District Miahuatlán, San Jerónimo Coatlán, A. Campos V. \& R. Torres C. 1573 (FCME, MEXU, MO), A. Campos V. \& R. Torres C. 1585 (MEXU, MO), R. Torres C. \& A. Campos V. 10789 (MEXU). District Mixe, Santiago Zacatepec, J. Santana C. \& L. Pacheco 784 (UAMIZ(x5)); Totontepec Villa de Morelos, E. Ramírez G. \& P. Ramírez C. 27 (IEB, MEXU), J. Rivera R. JR1058 (MEXU(x2)), J. Rivera R. \& G. J. Martin 1718 (MEXU, UAMIZ). District Tuxtepec, San Felipe Usila, A. Rincón G., J. Meave del C., C. Gallardo H., A. Otero A. \& L. Lozada P. 234 (MEXU, XAL). District Villa Alta, San Juan Juquila Vijanos, D. H. Lorence \& R. Cedillo T. 4085 (ENCB(x2), MEXU(x2)), X. Munn, R. Rivera \& N. Sánchez 78 (MEXU(x3)); San Melchor Betaza, R. Torres C. \& R. Cedillo T. 2036 (MEXU).

\section{Excluded names and taxa}

The following names and/or taxa have been cited for (Palací, 1997; Smith, 1974, 1977, 1979) or suspected to be in Oaxaca, but by the reasons below exposed we have decided to exclude them.

Aechmea galeottii Baker: The type specimens of $A$. galeottii (BR, P) correspond to A. lueddemanniana (K. Koch) Mez.

Aechmea tillandsioides (Mart. ex Schult. \& Schult. f.) Baker var. kienastii (E. Morren ex Mex) L. B. Sm.: We did not find sufficient differences to separate this variety from the type variety, so we consider this name as a synonym of $A$. tillandsioides.

Androlepis skinneri (K. Koch) Brongn. ex Houllet: This species has been recorded from the municipio of Uxpanapa in the state of Veracruz, a few kilometers north of the Oaxacan border, because of this fact we expect to find it in the municipio of Santa María Chimalapa in Oaxaca. Further collections may reveal its presence in the state.

Billbergia mexicana Mez: This name is a synonym of $B$. pallidiflora Liebm.

Billbergia oaxacana Matuda: This species is in fact $B$. pallidiflora Liebm.

Bromelia alsodes H. St. John: The specimens from Oaxaca identified with this name are in fact $B$. pinguin L.

Catopsis hahnii Baker: a carefully examination of the type (K!, P!) of this species revealed that is in fact a synonym of C. paniculata E. Morren. Some other specimens identified as C. hahnii belong to C. oerstediana.

Greigia oaxacana L. B. Sm.: This name is a synonym of G. juareziana.

Hechtia macdougallii L. B. Sm.: This name is here considered as a synonym of $H$. rosea. 
Hechtia stenopetala Klotzsch: all the material cited by Smith (1974) as H. stenopetala belongs in fact to H. pringlei B. L. Rob. \& Greenm. H. stenopetala is endemic to Veracruz.

Pepinia punicea (Scheidw.) Brongn. ex André: This species has been collected in the municipio of Uxpanapa In the state of Veracruz, a few kilometers north of the Oaxacan border, so we expect to find it in the municipio of Santa María Chimalapa in Oaxaca. Further collections may reveal its presence in the state.

Pitcairnia cylindrostachya L. B. Sm.: Plants from Oaxaca identified with this name belong to $P$. ringens Klotzsch.

Pitcairnia karwinskyana Schult. \& Schult. f.: Plants from Oaxaca identified with this name belong to $P$. ringens Klotzsch.

Tillandsia andrieuxii (Mez) L. B. Sm.: The specimens from Oaxaca identified with this name belong to T. macdougallii L. B. Sm.

Tillandsia argentea Griseb.: This species, as pointed out in Till (1990; 1992) inhabits only the Antilles (Cuba and Jamaica). The Mexican plants belong to T. fuchsii Till.

Tillandsia bartramii Elliott: The Oaxacan plants identified as T. bartramii belong to $T$. hammeri Rauh \& Ehlers (Conzatti 1743 (MEXU!, US)), and to T. ortgiesiana E. Morren ex Mez (T. Macdougall s. $n$ (US)).

Tillandsia benthamiana Baker: This name has been used for T. erubescens Schltdl., however we have not seen specimens of this species from Oaxaca. All the material we have seen belongs to T. macdougalli L. B. Sm.

Tillandsia circinnata Schltdl.: This name represents an ancient identification of T. paucifolia Baker.

Tillandsia dasyliriifolia Baker: This species is endemic to Yucatán peninsula. The Oaxacan specimens identified with this name belong to T. huamelulaensis Ehlers or to T. makoyana Baker.

Tillandsia fasciculata Sw. var. clavispica Mez: the only Oaxacan specimen identified with this name belongs in fact to T. callichroma L. Hrom.

Tillandsia fasciculata Sw. var. densispica Mez: the Oaxacan specimens identified with this name belong in fact to $T$. fasciculata Sw.

Tillandsia mexicana L. B. Sm.: With this name were identified Oaxacan specimens of T. callichroma L. Hrom. and T. dugesii Baker.

Tillandsia mooreana L. B. Sm.: The reports of this species from Oaxaca belong to Tillandsia kalmbacheri Matuda. T. mooreana is known only from the north of Mexico.

Tillandsia ponderosa L. B. Sm.: The only Oaxacan specimen reported with this name ( $T$. Macdougall 334) belongs in fact to T. yunckeri.

Tillandsia pueblensis var. glabrior L. B. Sm.; the correct name for this taxon is T. glabrior (L. B. Sm.) López-Ferrari, Espejo \& I. Ramírez.

Tillandsia remota Wittm.: This species in known only from Guatemala and Chiapas, near Guatemalan border. The plants from Oaxaca belong to T. pseudosetacea Ehlers \& Rauh.

Tillandsia rodrigueziana Mez: Plants identified with this name belong in fact to T. polita L. B. Sm. var. elongata Ehlers.

Tillandsia vicentina Standl. var. glabra L. B. Sm.: Plants identified with this name belong to T. polita L. B. Sm. var. elongata Ehlers. 
Vriesea ovandensis Matuda: The correct name for this species is: Werauhia pycnantha (L. B. Sm.) J. R. Grant.

Vriesea vanhyningii L. B. Sm.: The correct name for this species is: Werauhia vanhyningii (L. B. Sm.) J. R. Grant.

Vriesea werckleana Mez: The correct name for this species is: Werauhia werckleana (Mez) J. R. Grant. 